\title{
The management of multiple submissions in parallel systems: the fair scheduling approach
}

\author{
Vinicius Gama Pinheiro
}

THESIS PRESENTED

TO THE

Institute of MATHEMATICS AND STATistics

OF

UNIVERSITY OF SÃO PAULO

FOR

OBTAINING

THE

Doctorate Degree in SCIENCES

\author{
Program: Computer Science \\ Advisor: Prof. Dr. Alfredo Goldman \\ Co-advisor: Prof. Dr. Denis Trystram
}

During the development of this work,

the author received financial support from CAPES/COFECUB

São Paulo, April 14, 2014 


\section{The management of multiple submissions in parallel systems: the fair scheduling approach}

This thesis version contains all the changes and corrections that were suggested by the Jury Committee during the defense of the original version, held at February 14, 2014. A copy of the original version is available at the Institute of Mathematics and Statistics of University of São Paulo.

Jury Committee:

- Prof. Dr. Alfredo Goldman (advisor)- IME-USP, Brazil

- Prof. Dr. Denis Trystram - Grenoble Institute of Technology, France

- Prof. Dr. Evripidis Bampis - Université Pierre et Marie Curie, France

- Prof. Dr. Luiz Fernando Bittencourt - UNICAMP, Brazil

- Prof. Dr. Frédéric Suter - INP2P3 Computing Center, France 
For Cécile Zozzoli, a true life partner, a beautiful woman, and a loving wife whose affection, comprehension, and devotion inspire me and bring joy to my life. 


\section{Acknowledgements}

This thesis represents not only a technical piece of work but also a personal overcoming, given the challenges that appeared on my way, some anticipated, others completely unexpected. Many people contributed for my success in this endeavor.

First, I would like to thank Prof. Denis Trystram for being my advisor, specially during my stay in France. I am very grateful for all the guidance he provided me during my studies, for his patience, and also for receiving me so well in your research group.

To my Brazilian advisor, Prof. Alfredo Goldman, thank you for all the support, friendship and patience. And for always trusting me.

To Prof. Krzysztof Rzadca, from University of Warsaw, thank you for the great discussions and academic partnership in writing papers. Oh, and thank you for having lent me your bike! I really enjoyed the time I spent in Warsaw.

Another professors also helped me along the way: Prof. Arnaud Legrand, Prof. Daniel Cordeiro, and Prof. Daniel Batista. Thank you all for the fruitful discussions and suggestions.

To my LIG mates Emílio Francesquini, Marcio Castro, and Gabriel Duarte: my stay in France was way more pleasant beside you guys. Also, a big thank you for Valentin Reis and Joseph Emeras, great study partners.

To my LCPD mates Paulo Meirelles, Gustavo Duarte, Raphael Cobe, Beraldo Leal, and Marcio Vinicius: thank you for the great moments in the lab and on the campus.

Last, but not least important, to Cécile Zozzoli, my dear wife, and to Getúlio, Marilene, Rúbia and Jacqueline Pinheiro, my parents and sisters, beloved ones. 


\section{Abstract}

The High Performance Computing community is constantly facing new challenges due to the ever growing demand for processing power from scientific applications that represent diverse areas of human knowledge. Parallel and distributed systems are the key to speed up the execution of these applications as many jobs can be executed concurrently. These systems are shared by many users who submit their jobs over time and expect a fair treatment by the scheduler.

The work done in this thesis lies in this context: to analyze and develop fair and efficient algorithms for managing computing resources shared among multiple users. We analyze scenarios with many submissions issued from multiple users over time. These submissions contain several jobs and the set of submissions are organized in successive campaigns. In what we define as the Campaign Scheduling model, the jobs of a campaign do not start until all the jobs from the previous campaign are completed. Each user is interested in minimizing the flow times of their own campaigns. This is motivated by the user submission behavior whereas the execution of a new campaign can be tuned by the results of the previous campaign.

In the first part of this work, we define a theoretical model for Campaign Scheduling under restrictive assumptions and we show that, in the general case, it is NP-hard. For the single-user case, we show that an approximation scheduling algorithm for the (classic) parallel job scheduling problem also delivers the same approximation ratio for the Campaign Scheduling problem. For the general case with multiple users, we establish a fairness criteria inspired by time sharing. Then, we propose a scheduling algorithm called FairCamp which uses campaign deadlines to achieve fairness among users between consecutive campaigns. 
The second part of this work explores a more relaxed and realistic Campaign Scheduling model, provided with dynamic features. To handle this setting, we propose a new algorithm called OStrich whose principle is to maintain a virtual time-sharing schedule in which the same amount of processors is assigned to each user. The completion times in the virtual schedule determine the execution order on the physical processors. Then, the campaigns are interleaved in a fair way. For independent sequential jobs, we show that OStrich guarantees the stretch of a campaign to be proportional to campaign's size and to the total number of users. The stretch is used for measuring by what factor a workload is slowed down relatively to the time it takes to be executed on an unloaded system.

Finally, the third part of this work extends the capabilities of OStrich to handle parallel jobs. This new version executes campaigns using a greedy approach and uses an eventbased resizing mechanism to shape the virtual time-sharing schedule according to the system utilization ratio.

Keywords: campaign, multi-user, fairness, scheduler 


\section{Résumé}

La communauté de Calcul Haute Performance est constamment confrontée à de nouveaux défis en raison de la demande toujours croissante de la puissance de traitement provenant d'applications scientifiques diverses. Les systèmes parallèles et distribués sont la clé pour accélérer l'exécution de ces applications, et atteindre les défis associés car de nombreux processus peuvent être exécutés simultanément. Ces systèmes sont partagés par de nombreux utilisateurs qui soumettent des tâches sur de longues périodes au fil du temps et qui attendent un traitement équitable par l'ordonnanceur.

Le travail effectué dans cette thèse se situe dans ce contexte: analyser et développer des algorithmes équitables et efficaces pour la gestion des ressources informatiques partagés entre plusieurs utilisateurs. Nous analysons les scénarios avec de nombreux soumissions issues de plusieurs utilisateurs. Ces soumissions contiennent un ou plusieurs processus et l'ensemble des soumissions sont organisées dans des campagnes successives. Dans ce que nous appelons le modèle d'ordonnancement des campagnes les processus d'une campagne ne commencent pas avant que tous les processus de la campagne précédente soient terminés. Chaque utilisateur est intéressé à minimiser la somme des temps d'exécution de ses campagnes. Cela est motivé par le comportement de l'utilisateur tandis que l'exécution d'une campagne peut être réglé par les résultats de la campagne précédente.

Dans la première partie de ce travail, nous définissons un modèle théorique pour l'ordonnancement des campagnes sous des hypothèses restrictives et nous montrons que, dans le cas général, il est NP-difficile. Pour le cas mono-utilisateur, nous montrons que l'algorithme d'approximation pour le problème (classique) d'ordonnancement de processus parallèles fournit également le même rapport d'approximation pour l'ordonnancement 
viii

des campagnes. Pour le cas général avec plusieurs utilisateurs, nous établissons un critère d'équité inspiré par une situation idéalisée de partage des ressources. Ensuite, nous proposons un algorithme d'ordonnancement appelé FairCamp qui impose des dates limite pour les campagnes pour assurer l'équité entre les utilisateurs entre les campagnes successives.

La deuxième partie de ce travail explore un modèle d'ordonnancement de campagnes plus relâché et réaliste, avec des caractéristiques dynamiques. Pour gérer ce cadre, nous proposons un nouveau algorithme appelé OStrich dont le principe est de maintenir un ordonnancement partagé virtuel dans lequel le même nombre de processeurs est assigné à chaque utilisateur. Les temps d'achèvement dans l'ordonnancement virtuel déterminent l'ordre d'exécution sur le processeurs physiques. Ensuite, les campagnes sont entrelacées de manière équitable. Pour des travaux indépendants séquentiels, nous montrons que OStrich garantit le stretch d'une campagne en étant proportionnel à la taille de la campagne et le nombre total d'utilisateurs. Le stretch est utilisé pour mesurer le ralentissement par rapport au temps qu'il prendrait dans un système dédié.

Enfin, la troisième partie de ce travail étend les capacités d'OStrich pour gérer des tâches parallèles rigides. Cette nouvelle version exécute les campagnes utilisant une approche gourmande et se sert aussi d'un mécanisme de redimensionnement basé sur les événements pour mettre à jour l'ordonnancement virtuel selon le ratio d'utilisation du système.

Mots-clés: campaigne, multi-utilisateur, fairness, ordonnanceur. 


\section{Contents}

List of Figures $\quad$ xiii

1 Introduction $\quad 1$

1.1 Fairness matters . . . . . . . . . . . . . . . . . 3

1.2 Multiple submissions as job campaigns . . . . . . . . . . . . . . 5

1.3 Thesis outline and contributions . . . . . . . . . . . . . 7

2 Background $\quad 9$

2.1 Classical scheduling theory $\ldots \ldots \ldots \ldots$. . . . . . . . . . 10

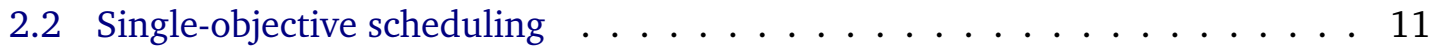

2.3 Multi-objective scheduling . . . . . . . . . . . . . . . . 15

2.4 Fairness in scheduling $\ldots \ldots \ldots \ldots \ldots \ldots \ldots$

3 The Campaign Scheduling Problem 31

3.1 The campaign model . . . . . . . . . . . . . . . . . . . . 32

3.2 Related models . . . . . . . . . . . . . . . . . . . . . . 32

3.2 .1 Bag-of-Tasks . . . . . . . . . . . . . . . . . 33

3.2.2 Parameter sweep applications. . . . . . . . . . . . . . . . . . 33

3.2 .3 BSP model. . . . . . . . . . . . . . . . . . . . 33

3.2 .4 Similarities and applicability . . . . . . . . . . . . 34

3.3 Definitions and notation $\ldots \ldots \ldots \ldots \ldots$

3.4 Offline scheduling of single user's campaigns . . . . . . . . . . . . . . 38

3.5 Offline scheduling with multiple users $\ldots \ldots \ldots$. . . . . . . . . 41 
3.6 Online scheduling with multiple users . . . . . . . . . . . . . 43

3.6.1 Measuring fairness by max-stretch . . . . . . . . . . . 43

3.6.2 FCFS in multi-user systems . . . . . . . . . . . . . . 44

4 Fair online schedules: constrained scenario $\quad 47$

4.1 The FairCamp online algorithm . . . . . . . . . . . . . . . . 48

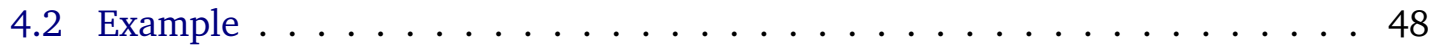

4.3 Algorithm description . . . . . . . . . . . . . . . . . . 49

4.4 Feasibility of FairCamp . . . . . . . . . . . . . . . . . 51

4.5 Theoretical analysis . . . . . . . . . . . . . . . . . 52

4.6 Simulations . . . . . . . . . . . . . . . . . . 54

5 Fair online schedules: dynamic scenario $\quad 57$

5.1 The OStrich online algorithm . . . . . . . . . . . 58

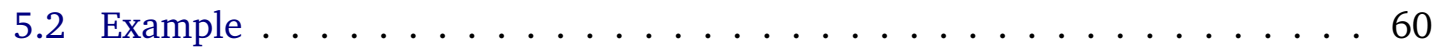

5.3 Theoretical analysis . . . . . . . . . . . . . . . . . 63

5.3 .1 Worst-case bound . . . . . . . . . . . . . . 63

5.3 .2 Tightness ........................ 67

5.4 Analysis of campaigns in workloads . . . . . . . . . . . . . . . 67

5.4 .1 Workload modeling . . . . . . . . . . . . . . . 68

5.4 .2 Example . . . . . . . . . . . . . . . . . 69

5.4 .3 Interval graphs for job dependencies $\ldots \ldots \ldots 70$

5.4.4 Campaigns with dependencies: a formal model . . . . . . . . . 71

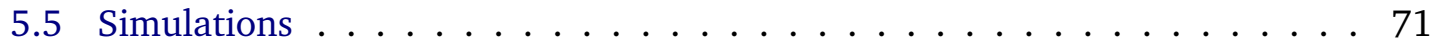

$\begin{array}{lll}6 & \text { Scheduling parallel jobs with OStrich } & 79\end{array}$

6.1 Parallel OStrich . . . . . . . . . . . . . . . . . . . . . . 79

6.1 .1 Event based resizing $\ldots \ldots \ldots$. . . . . . . . . . 80

6.2 Algorithm description $\ldots \ldots \ldots \ldots \ldots \ldots$

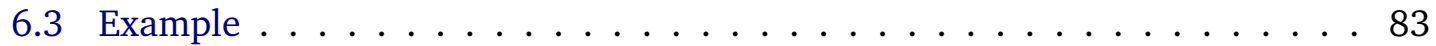

6.4 Comparison with existing scheduling strategies $\ldots \ldots$. . . . . . . . . 84 
6.5 Theoretical analysis . . . . . . . . . . . . . . . . . 87

6.5 .1 Worst-case bound . . . . . . . . . . . . . . . 88

7 Conclusion and ongoing work $\quad 91$

7.1 Work contributions and perspectives . . . . . . . . . . . . . . 92

Bibliography 


\section{List of Figures}

1.1 Campaign Scheduling with 2 users (user 1 in light gray, user 2 in dark gray) 6

2.1 Example of a Gantt chart with 3 machines and 7 jobs $\ldots \ldots \ldots$. . . . . . 10

2.2 List scheduling example with independent jobs $\ldots \ldots \ldots$. . . . . . . 13

2.3 LPT Gantt charts . . . . . . . . . . . . . . . . . . . . 14

2.4 Pareto set for the set of solutions $X=\left\{X_{1}, X_{2}\right\} \ldots \ldots \ldots \ldots$

2.5 Approximability bound for MUSP . . . . . . . . . . . . . . . . 18

2.6 Pareto optimality for MUSP (user 1 in light gray, user 2 in dark gray) . . . 20

2.7 Space-sharing schedule example with 3 users . . . . . . . . . . . . . . 22

2.8 Time-sharing schedule example with 3 users . . . . . . . . . . . . . . 22

2.9 Examples of space-share and time-share schedules with 2 users and corresponding Pareto solutions . . . . . . . . . . . . . . . . . 23

2.10 FCFS worst-case ratio with 2 users $\ldots \ldots \ldots$. . . . . . . . . . 25

2.11 LPT starvation with 2 users $\ldots \ldots \ldots \ldots$

2.12 SPT starvation with 2 users $\ldots \ldots \ldots \ldots \ldots$

2.13 Example of Hadoop Fair Scheduler pools with 3 users . . . . . . . . . . . 28

2.14 Example of Slurm schedule with 3 users using Fair-share factor and $\Delta t=2$. 29

3.1 Campaign submission and execution on a 4 processor system . . . . . . 32

3.2 Campaign Scheduling with 2 users (user 1 in light gray, user 2 in dark gray) 36 
3.3 FCFS competitiveness. $k=2, \sigma=2$, user 1 in light gray, user 2 in dark gray. Max-stretch is $(2 p+2) / 2 \approx p$ (for large $p$ ); the optimal max-stretch is $(2 p+2) /(2 p) \approx 1$ (for large $p$ ). The faded campaign represents the optimal position of the second campaign for user $2 \ldots \ldots$. . . . . . . . . 45

4.1 Deadlines with $k=2$ and multiple campaigns . . . . . . . . . . . . . 48

4.2 Example of schedule constructed by FairCamp (user 1 in light grey and user 2 in dark grey $) \ldots \ldots$. . . . . . . . . . . . . . . . . . . . . 49

4.3 FCFS vs FairCamp; each point is an average over $10^{3}$ instances; error bars denote $95 \%$ confidence intervals . . . . . . . . . . . . . . . 54

4.4 Max-stretch distribution for FCFS and FairCamp with 20 users . . . . . . . . 55

5.1 An example of the virtual and real schedule generated by the OStrich algorithm with 3 users $\ldots \ldots \ldots \ldots$. . . . . . . . . . . . 61

5.2 Analysis of OStrich: bound for the campaign stretch . . . . . . . . . . . 65

5.3 An example of a campaign with seven jobs according to the MAX algorithm $\quad 70$

5.4 A Directed Acyclic Graph (DAG) illustrating dependencies inside the campaign $($ same campaign of Figure 5.3$) \ldots \ldots$. . . . . . . . 70

5.5 Campaign stretch values distribution (original log: Maui, FCFS) . . . . . . . 73

5.6 Campaign stretch values distribution (OStrich) $\ldots \ldots \ldots$. . . . . . . . 74

5.7 System resources utilization for whole trace (original log: Maui, FCFS) . . 74

5.8 System resources utilization for the whole trace (OStrich) $\ldots \ldots \ldots$. . . . 75

5.9 System resources utilization for the extract (original log: Maui, FCFS) . . . . 75

5.10 System resources utilization for the extract (OStrich, with job dependencies) 76

5.11 System resources utilization for the extract (OStrich, no dependencies) . . 76

5.12 Ostrich vs FCFS: stretch values by intervals . . . . . . . . . . . . . . . 77

5.13 Ostrich vs FCFS: max campaign stretch mean per user profile $\ldots \ldots$. . . 78

6.1 Delay problem between real and virtual schedule . . . . . . . . . . . . . 80

6.2 Delay correction using a resized virtual schedule $\ldots \ldots$. . . . . . . . 81

6.3 Virtual and real schedule generated by the OStrich algorithm with 3 users . 84 
6.4 FCFS example with 2 users $\ldots \ldots \ldots \ldots \ldots$

6.5 Space share example with 2 users $\ldots \ldots \ldots . \ldots . \ldots 66$

6.6 OStrich example with 2 users . . . . . . . . . . . . . . 87

6.7 Analysis of OStrich with parallel jobs: stretch bound of a campaign execution 90 
"Equals should be treated equally and unequals unequally - but in proportion to their relevant

\section{Chapter 1}

similarities and differences."

Someone on Aristotle's thoughts

\section{Introduction}

Since the second half of the last century, the hardware and software industries conducted several technological advances that helped to enlarge the boundaries of computer science in a wide variety of fields such as structural analysis, oil exploration, atmospheric simulation, weather forecast, seismic data processing, defense applications, chemistry and genetic analysis [ER06]. The emergence and popularization of parallel computing was one of the major factors that contributed to this phenomena.

Parallelism is a concept that arose on the early days of computer science as a way for speeding up the execution time of processes. It embraces a large variety of techniques used to split jobs in several parts to be computed by interconnected processor units. It became very popular during the eighties due to the appearance of the first commercially available general purpose parallel machines. However, they were expensive machines and not easily accessible [BTEP00]. The arrival of powerful microprocessors used in workstations provided high computation power at reasonable costs and was a major factor for the emergence of parallel high performance computers and systems like clusters, grids, supercomputers and desktop grids. Currently, HPC systems with a cluster architecture represent more than 83\% of the systems on the list of the 500 most powerful systems in the world [Sit] ${ }^{1}$.

Nevertheless, despite the fact that High Performance Computing systems (HPC systems) are cheaper and easier to obtain than before, they are hosted by organizations rather

\footnotetext{
${ }^{1}$ From the time of this writing, this list was last updated in June 2013.
} 
than individuals. They require specialized personnel and robust infrastructures whose management is complex and time consuming. Hence, these systems are commonly shared by many users and projects who compete for the usage of the resources in order to execute their jobs.

Typically, the management of resources is handled by local and distributed resource managers through a consensual scheduling policy. Even with all the popularity achieved by HPC systems, and the computational power they aggregate, scheduling management remains one of the main challenges due to the complexity of the majority of scheduling problems $[\mathrm{BK}]$. It becomes even more complex if users desire fairness and performance guarantees. The management of umbrella projects in BOINC platform is a good example where these issues show their pertinence.

BOINC [And04] is a platform for volunteer computing, through which volunteers can donate their machines' CPU idle time to scientific projects. It comprises over 580,000 hosts that deliver more than 2,300 TeraFLOP per day to several projects. BOINC projects usually have hundreds of thousands of CPU-bound jobs. These projects are traditionally interested in overall throughput, i.e., maximize the total number of jobs completed per day. Generally, the jobs submitted through BOINC are independent and preemptive.

Each project has its own server which is responsible for distributing work units to clients as well as recovering and validating results. But the tasks of deploying and maintaining a BOINC server can represent a great burden in terms of time and money. Umbrella projects appeared as a way to address this problem. Those are multi-user projects that share the same infrastructure, each one hosting their scientific sub project. Besides the economy advantage, this solution provides a much larger number of volunteers to every project than if they had deployed their own server. But this solution also creates new challenges.

In such umbrella projects, it is common that the demand for computing power exceeds the available supply, therefore a mechanism to split the supply among users is needed. Nevertheless, each sub project has its own goals and distinct processing needs that can be represented by objective functions. In the past, most users were throughput-oriented but popularization of those systems attracted other types of users. Nowadays, response-time 
users are increasingly common [DLG11]. Their jobs are divided into successive batches of independent jobs released sequentially over time. For such users, throughput is not meaningful as they are more interested in minimizing the time each campaign takes to be executed.

In this thesis, we study how to improve fairness between response-time users in parallel systems. We model variants of this problem according to different user submission dynamics and job constraints. We provide solutions to each case and, using theoretical tools, we study how the trade-off between fairness and user performance is met.

\subsection{Fairness matters}

Users are human beings and, as such, they are sensitive to the way resources are shared in their social circles. The equity theory developed in 1965 by John Stacey Adams in his seminal article entitled "Inequity in Social Exchange" [Ada65], argues that, in social settings, individuals (e.g. users) are selfish in general, meaning that they look only for their own objectives and reject the idea of not being treated fairly when compared to the other individuals. According to this work, inequity exists between two persons A and B when there is a difference between the ratio of A's outcomes to A's inputs and the ratio of B's outcomes to B's inputs. This may happen in a direct exchange relationship between them or when both are in an indirect exchange relationship with a third party and one compares himself to the other.

This theory is mainly based on two concepts relating to the perception of justice and injustice. The first one, called "relative deprivation" is a sociological concept developed by Stouffer et al. [SLL ${ }^{+}$49] from his survey over American soldiers during World War II. The authors observed that more educated soldiers were less satisfied with their status than less educated ones, despite the fact that the former had better career opportunities in the army. This paradox was explained by assuming that better-educated men, who had made more investments in their formation, had higher levels of aspiration and, therefore, that they were relatively deprived of status based on what they achieved. In short, this concept states that the degree of satisfaction of one person is closely related to his/her expectations. 
The second one, called distributive justice, is roughly defined as the perceived fairness in the way costs and rewards are shared within a group. More formally, distributive justice is achieved between two group members when:

$$
\frac{\text { A's rewards - A's costs }}{\text { A's investments }}=\frac{\text { B's rewards - B's costs }}{\text { B's investments }}
$$

In other words, this concepts states that the justice perceived by an individual is not only measured by his/her own ratio of profits to investments but, more importantly, the relation between ratios within a group. Some members can feel unfairly treated if they perceived that their ratio of profits is smaller than the other, even if it corresponds to their expectations.

Relative deprivation and/or lack of distributive justice are also applied to users sharing the resources of a system. They form a group with conflicting interests in an indirect exchange relationship with a third party, in this case, the scheduler. The decisions taken by the scheduler impact on the level of satisfaction experienced by each user. They will likely to compare their "rewards" or, more appropriately to this domain, resource allocations (e.g. processing share, allocated memory, etc.) to those of another and become envy of others anytime they feel deprecated.

Ideally, users should not envy their counterparts in a shared system. The notion of "envy-freeness" appeared in the book "Puzzle-Math" (1958) [GS58] from the physicists Gamow and Stern. For an algorithm to be envy-free, each user must prefer to keep their own allocations to swapping with other users.

Fair division of resources is also discussed in a very recent article by Procaccia entitled "Cake Cutting: Not Just Child's Play" [Pro13] where the author invites the computer scientists to dwell on this problem. This article surveys several cake cutting algorithms supporting that they can give insights that can be applied on the allocation of computational resources. It also discuss the notion of "envy-freeness" [GS58] and how it is addressed by existing theoretical models. However, it is very important to stress that, unlike what will be seen in this thesis, these actual models do not encompass dynamic features like users joining or leaving the system and online submissions.

Some of the concepts presented on this section will be adapted in the next chapter 
when discussing about fair division for scheduling tasks from multiple users. On the next section, it is presented the exchange nature (i.e. the interactive process) between users and scheduler that drives all the analysis present on this thesis.

\subsection{Multiple submissions as job campaigns}

In this thesis, the problem of multiple submissions on parallel system is narrowed to the notion of Campaign Scheduling. The campaign scheduling problem models a submission pattern typically found in parallel systems used for scientific research: the user submits a set of jobs, analyzes the outcomes and then resubmits another set of jobs [ZAT05, ZF12]. In other words, the campaigns are sets of jobs issued from a user and they must be scheduled one after the other since the submission of a new campaign depends on the outcome of the previous one. Reflecting that, the maximum number of campaigns being simultaneously executed in the system is at most the number of users. As this pattern is an interactive process, the objective of each user is to minimize the time each campaign spends in the system, namely the campaign's flow time. The sooner a campaign finishes, the sooner the user will be ready to submit the next one.

In a campaign, the jobs can be dependent or independent, sequential or parallel. The flow time is defined as the time interval between the submission and the completion of the last task of a campaign. To give an example, Figure 3.2 illustrates the submission of 4 campaigns from two users, 1 and 2, in a parallel system. The users are represented by different shades of gray. The call-outs represent the campaigns' submissions, the tracks represent the campaigns' execution periods (sometimes preceded by lines that represent wait times) and the arrows symbolize the precedence relations between campaigns.

In this thesis, it is shown how this campaign model can be explored in a better way than classical and actual fair share algorithms. We propose solutions that make a compromise between fairness and execution performance.

Each solution is geared for different scenarios. For the more restrict case, the FairCamp algorithm is proposed. This is a scheduling algorithm which uses campaign deadlines to achieve fairness among users between consecutive campaigns. It assumes the number of 


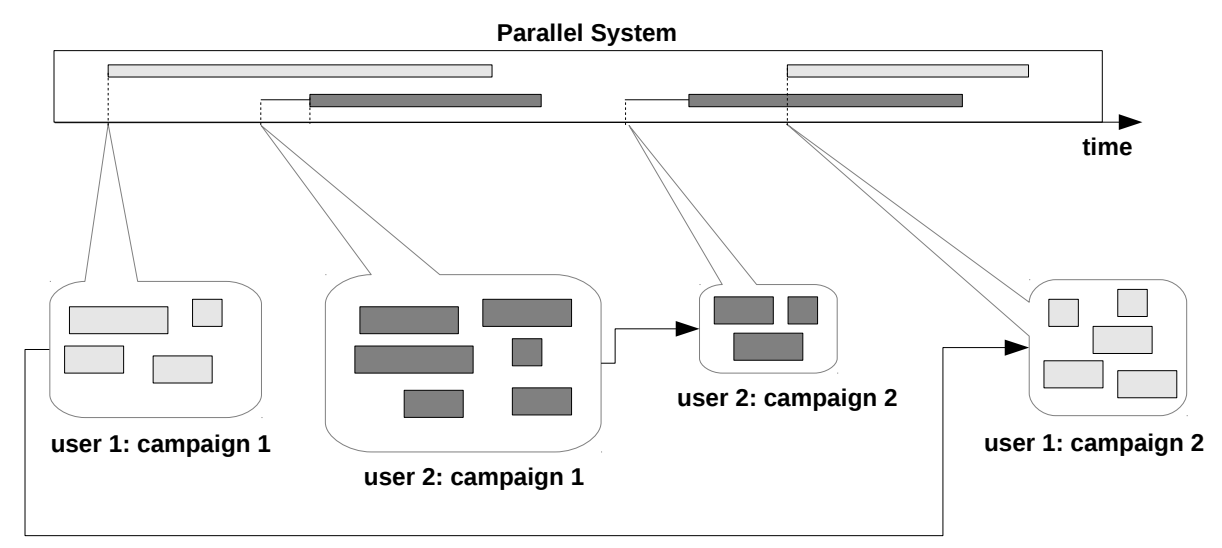

Figure 1.1: Campaign Scheduling with 2 users (user 1 in light gray, user 2 in dark gray)

users to be static and no time interval between two consecutive campaigns. We prove that FairCamp increases the flow time of each user by a factor of at most $k \rho$ compared with a machine dedicated to the user, with $k$ being the number of users and $\rho$ being the approximation factor of the algorithm used to schedule jobs within campaigns. We also prove that FairCamp is a $\rho$-approximation algorithm for the maximum stretch.

Beyond FairCamp, and targeting more dynamic scenarios, the OStrich was proposed. This algorithm is suitable for a more realistic setting where the number of users changes and breaks between campaigns are common. OStrich schedules the jobs according to a priority list where the priorities are determined by campaign's virtual completion time. This virtual completion time is defined as the time the campaign would take to complete in an ideal divisible load model and using a time-sharing scheduling strategy that assigns an equal share of processors to each competing user. However, OStrich does not assign actual processors to jobs in a time-sharing manner. Instead, the campaign with highest priority takes all the available processors.

Finally, in order to embrace campaigns with parallel jobs, an improved version of OStrich is proposed. This version maintains the mechanisms that are present in the sequential job version, but with further modifications to handle the idle gaps that may occur when scheduling parallel jobs. On becoming aware of these solutions, it is interesting to note that OStrich for parallel jobs is not only the more refined of them but also the more general 
since it is suitable for sequential jobs as well, needing only few adjustments. The choice of presenting the solutions and the related analysis according to an increasing level of refinement is justified: it allows to understand how the work evolved and it provides a better understanding of each mechanism embedded in the solutions.

\subsection{Thesis outline and contributions}

The rest of this work is organized as follows. In Chapter 2, we present the main notions that will be used as tools in the remaining of the thesis. First, we present some basic definitions about scheduling theory and single optimization. Then we discuss about multioptimization, focusing in multi-user scheduling problems. We also explore the concept of fairness depicted in the recent literature and how it is implemented in actual systems.

Chapter 3 is devoted to the description of the Campaign Scheduling problem, its modeling, and on which contexts it can be applied. We give an analysis of the offline problem for single and multi-user perspectives. Some formal results are obtained along with a solution. But, despite the fact that this setting can be applied in some real cases, it is not general enough to represent all the dynamics in user interaction with parallel systems. So, still in this chapter, we analyze the online problem regarding First-Come-First-Served (FCFS) as a basis of comparison.

Throughout chapters 4, 5 and 6 we analyze online settings of the Campaign Scheduling problem and we deliver solutions that are appropriate for each case.

In Chapter 4, we study a constrained scenario and we propose FairCamp, a scheduling algorithm which uses campaign deadlines to achieve fairness. We prove that FairCamp delivers response times that are distant from the optimal by at most $k \rho$ where $k$ is the number of users and $\rho$ is the approximation factor of the algorithm used to schedule the jobs inside a campaign. We also prove that FairCamp is a $\rho$-approximation algorithm for the maximum stretch with $k$ users.

Chapter 5 presents a new fair scheduling algorithm called OStrich whose principle is to maintain a virtual time-sharing schedule in which the same amount of processors is assigned to each user. The completion times in this virtual schedule determine the execution 
order on the physical processors. Then, the campaigns are interleaved in a fair way by OStrich. For independent sequential jobs, we show that OStrich guarantees the stretch of a campaign to be proportional to campaign's size and the total number of users.

Another version of OStrich is presented in Chapter 6. This version is suitable for parallel jobs where campaigns are executed using a greedy algorithm. The virtual time-sharing schedule is updated in an event driven fashion to handle idle spaces that may appear on the real scheduler.

Finally, at Chapter 7, we provide our conclusions and perspectives about future works, along with the contributions and accepted publications. 


\section{Chapter 2}

\section{Background}

This chapter covers the topics that form the basis of the work presented in this thesis.

In Section 2.1, we present some standard information about scheduling theory and some basic concepts and definitions. More specifically, we discuss about different types of jobs, parallel systems and how the scheduling of jobs can be represented in Gantt charts.

Section 2.2 presents the notation proposed by Graham et al. to classify scheduling problems and describes some classical problems that focus on optimizing a single objective. Some well known scheduling algorithms that are used throughout this work are introduced here, followed by analysis that help us to understand their behavior.

Section 2.3 goes one step further by describing some scheduling problems whose focus is on the optimization of many objectives. This set of problems is the target of another subjects of study like fairness and the concept of Pareto optimality, that are also presented. This section is also an overview about recent works that are associated with optimizing objectives from many users. In fact, it serves as a preamble of the main problem concerned in this thesis since it contains some insights about the limitations and challenges faced when trying to conciliate the demands of multiple users in parallel systems.

Finally, in Section 2.4, the concept of fairness and fair scheduling are discussed in more detail. We reason about the definition of fairness in resource sharing and we describe some fair scheduling mechanisms present in actual systems as well as some common metrics found in the literature. 


\subsection{Classical scheduling theory}

A schedule is an assignment of jobs to any physical device (like processors) over time. More formally, suppose that $m$ machines represented by the set $\mathcal{M}=\left(m_{1}, m_{2}, \ldots, m_{m}\right)$ have to process $n$ jobs represented by the set $\mathcal{J}=\left(J_{1}, J_{2}, \ldots, J_{n}\right)$. A schedule defines on which machine and at what time moment each job is allocated, in a way that each machine can be used by only one job at a given time. A Gantt chart is usually used to represent a schedule. The Figure 2.1 shows a machine-oriented Gantt chart with 3 machines and 7 independent jobs.

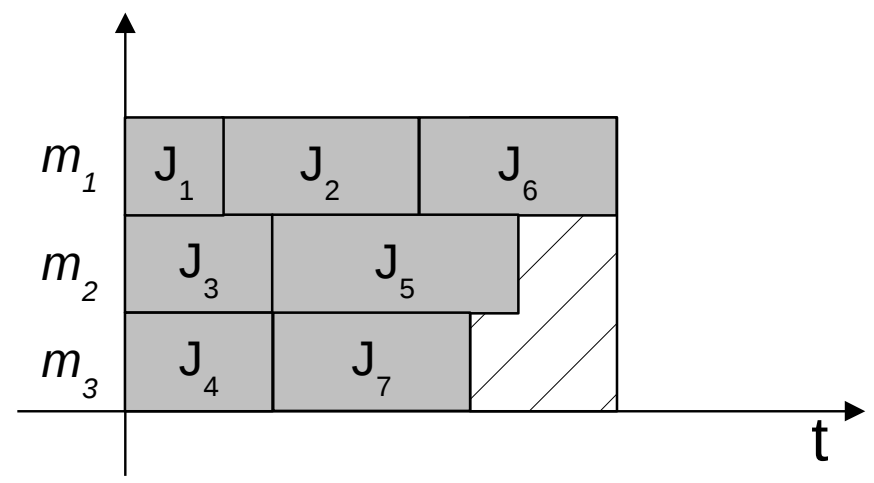

Figure 2.1: Example of a Gantt chart with 3 machines and 7 jobs

The machines can be classified as multi-purpose or parallel machines. If each job must be processed by a specific subset of $\mathcal{M}$, the machines are multi-purpose (or dedicated machines, if subsets are unitary). In opposite, machines can also be parallel machines, meaning that each job can be executed in any machine. Parallel machines, in turn, may be classified in three subtypes: identical, uniform, and unrelated processors [BTEP00]. Identical machines are indistinguishable with respect to processing of jobs. Uniform machines have different speeds, meaning that the speed ratio between two machines applies to all the jobs. For unrelated machines, the processing time of a job varies according to the processor allocated to it and this variation is particular to each job.

Jobs can be parallel or sequential. Sequential jobs require only one processor for their execution. For identical machines, a sequential job $J_{i}$ has a processing time $p_{i}$ and a release time $r_{i}$. The completion time is denoted by $C_{i}$. The processing time is the amount of time 
that a processor takes to execute the job. The release time is the time at which the job arrives to the system. This can also be referred as the submission time and we adopt this terminology in the remaining of this work.

Parallel jobs can be rigid, moldable, or malleable. Rigid jobs require a given fixed number of machines for their execution. This can also be referred as the size of the job, denoted by $q_{i}$. A moldable job is executed by a number of machines determined before the start of the job and this number is not changed until the job termination. The definition of malleable job is similar to the moldable job, with the exception that the number of machines can be changed at runtime.

Furthermore, the execution of jobs can be preemptive or non-preemptive, meaning that the processing may be interrupted and resumed a later time or not, respectively.

The basic problem of scheduling in distributed and parallel systems consists in efficiently sharing the resources among applications in order to optimize some criteria, mostly related to system performance like execution time of application jobs or resource utilization rate. A common problem, for example, is mapping a set of jobs onto the available processors of a system, selecting for each job the resource that would optimize the total completion time of the set.

Unfortunately, most of the problems studied in this area are NP-hard [LKB77, BK], which means that we can not find an optimal solution for those problems in polynomial time (unless $P=N P$ ). When we face a problem like this, the efforts must be directed in searching for algorithms whose solutions are distant from the optimal up to a guaranteed and small bound. These are called approximation algorithms.

In this work, we focus on approximation algorithms for the non-preemptive execution of sequential and parallel jobs on parallel identical machines.

\subsection{Single-objective scheduling}

Most of the problems studied in scheduling theory so far have a single objective. In these problems there is only one criteria to be optimized. In [GLLRK79], Graham et al. proposed a notation for describing and classifying scheduling problems. This notation consists of 
symbols organized in three fields $\alpha|\beta| \gamma$. The $\alpha$ field describes the machine environment (e.g. number of machines, if they are identical, uniform or unrelated, etc). The $\beta$ field is used to specify job characteristics such as size, processing time, precedence relations, and so on, or the hypothesis assumed on the schedule, like preemption for example. The $\gamma$ field describes the optimality criterion whereas classical examples are the total completion time of the jobs (also called makespan) and the sum of job completion times.

For instance, $P \| C_{\max }$ symbolizes the problem of minimizing the makespan - denoted by $C_{\max }$ - of jobs in a system composed of identical machines $(P)$. In this case, $\gamma=C_{\max }$ and $\alpha=P$. The $\beta$ field is empty, so the job characteristics are assumed to be standard, meaning that the jobs are sequential, independent, non-preemptive and have arbitrary size. This is one of the most basic scheduling problems and it is NP-hard [Ull75].

Many combinations of $\alpha|\beta| \gamma$ have been studied by researchers in the last decades in order to classify the complexity of various scheduling problems [Try12, LKB77, BK]. For the vast majority, no polynomial algorithm is known, thus it is reasonable to search for approximation algorithms. One of the main examples of such effort are List Scheduling algorithms proposed in 1966 by Graham [Gra66].

A list scheduling algorithm is based on a list of ready jobs. The principle of this class of algorithms is to pick a job from this list and schedule it on the resource that is available first. This action is repeatedly executed until all the jobs are scheduled. List Scheduling algorithms are proven to give solutions with good approximation ratios for many scheduling problems. In his seminal work [Gra66], Graham analyzed the $P \| C_{\max }$ problem. For this problem, list scheduling has a constant approximation factor of 2 and the proof is detailed next.

Theorem 2.1. Any list scheduling algorithm is a $(2-1 / m)$-approximation algorithm for $P \| C_{\max }$.

Proof. We need to show that the $C_{\max }$ delivered by a list scheduling algorithm is no larger than twice the optimal value (denoted by $C_{\max }^{*}$ ).

Let us denote starting time of a job $J_{j}$ as $s_{j}$, its processing time as $p_{j}$, and its completion time as $C_{j}$. Now, consider a schedule constructed by a list scheduling algorithm with $n$ jobs 


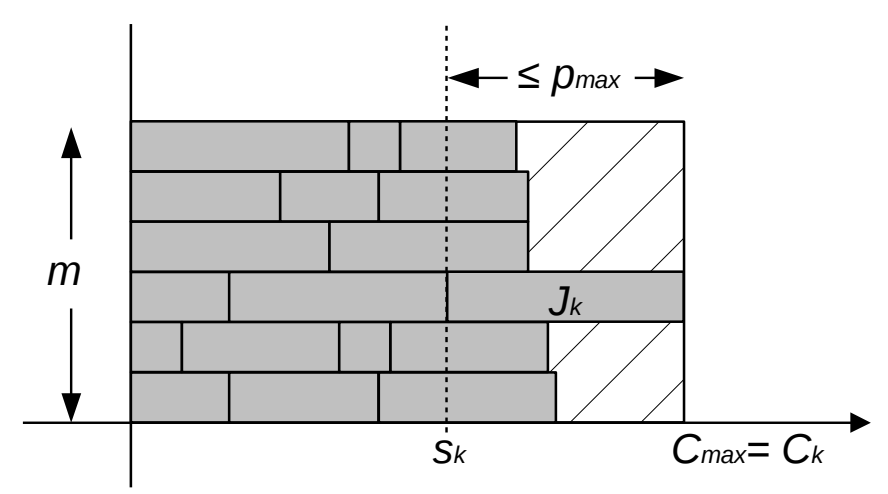

Figure 2.2: List scheduling example with independent jobs

where $J_{k}$ is the last job to complete. This schedule is outlined in figure 2.2. Let $C_{\max }$ be the makespan of the schedule, then, we have the $C_{\max }=C_{k}=s_{k}+p_{k}$, i.e. the completion time of $J_{k}$ is the makespan of the schedule.

Note that, if we want to minimize the makespan, the best possible solution would be to have the total work equally divided among the machines. So, the optimal makespan would be:

$$
C_{\max }^{*} \geq\left(\sum_{j=1}^{n} p_{j}\right) / m
$$

Another important observation is that, since $J_{k}$ is the last job, then no machine can be idle at any time prior to $s_{k}$, otherwise $J_{k}$ would have been started earlier. So, the workload composed of all the jobs apart from $J_{k}$ divided by the number of machines is equal to or greater than $s_{k}$ :

$$
s_{k} \leq\left(\left(\sum_{j=1}^{n} p_{j}\right)-p_{k}\right) / m=\left(\sum_{j=1}^{n} p_{j}\right) / m-p_{k} / m \leq C_{\max }^{*}-p_{k} / m .
$$

Adding $p_{k}$ to both sides:

$s_{k}+p_{k} \leq C_{\max }^{*}+p_{k}-p_{k} / m$

But, by definition, $C_{\max }=s_{k}+p_{k}$, then

$$
C_{\max } \leq C_{\max }^{*}+p_{k}(1-1 / m) \leq(2-1 / m) C_{\max }^{*} \leq 2 C_{\max }^{*}
$$




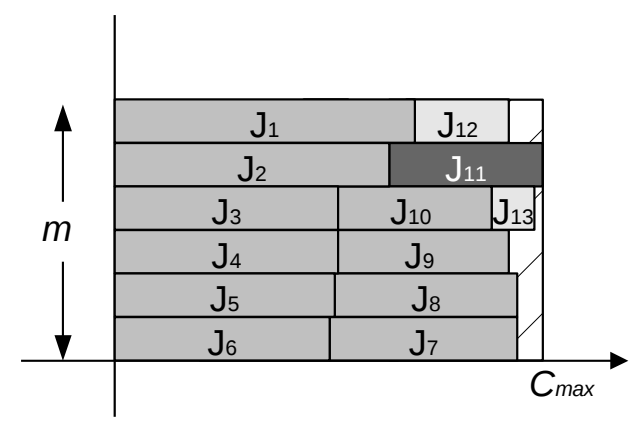

(a) Schedule built with LPT policy

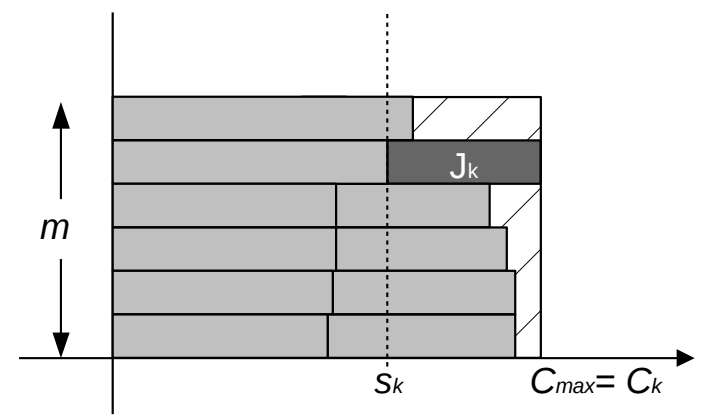

(b) The same schedule without job indexes $>k$

Figure 2.3: LPT Gantt charts

This bound was proved to be tight.

This is a generic, simple and yet powerful class of algorithms. Observe that in the context of independent tasks, list scheduling guarantees that the idle times are grouped at the end of the schedule. This allows us to improve the bound of 2 by leaving the small tasks to be scheduled at the end. That is the idea behind the LPT (Largest Processing Time) policy. The LPT is a list scheduling algorithm that schedules jobs in non-increasing order of processing times (see Figure 2.3a for an example). Graham showed that this algorithm has a performance ratio of $4 / 3$ for the $P \| C_{\max }$ problem [Gra69].

Theorem 2.2. $L P T$ is a $4 / 3$ approximation algorithm for $P \| C_{\max }$.

Proof. This analysis comes from the fact that this algorithm is optimal for a small number of jobs.

Again, consider a schedule constructed by a list scheduling algorithm with $n$ jobs where $J_{k}$ is the last job to complete. According to the way LPT builds the schedule, all the previous jobs $\left(J_{1}, J_{2}, \ldots, J_{k-1}\right)$ are larger (or equal) than $J_{k}$ and all the subsequent jobs 
$\left(J_{k+1}, J_{k+2}, \ldots, J_{n}\right)$ are smaller (or equal) than $J_{k}$ (Figure 2.3a). Removing the subsequent jobs from the schedule (Figure 2.3b), there are two cases to be analyzed.

First, $C_{\max }^{*}<3 p_{k}$.

In this case, one of the previous jobs is forcibly larger than $J_{k}$ and all the machines have 2 jobs at maximum to execute. Hence, it is not difficult to demonstrate that the solution is optimal.

Second, $C_{\max }^{*} \geq 3 p_{k}$.

In this case, let us decompose the schedule in two "areas", one from 0 up to $s_{k}$, completely filled by job activity, and another from $s_{k}$ to $C_{k}$, that mixes job activity with idle times. In the worst case, the total idle time (denoted by $S_{i d l e}$ ) is bounded by $(m-1) p_{k}$. So,

$$
\begin{aligned}
& C_{\text {max }}=\sum_{j=1}^{n} p_{j} / m+S_{i d l e} / m \leq C_{\text {max }}^{*}+(m-1) p_{k} / m . \\
& \text { As } p_{k} \leq C_{\text {max }}^{*} / 3, \\
& C_{\text {max }} \leq C_{\text {max }}^{*}+(m-1) C_{\text {max }}^{*} / 3 m \leq(4 / 3-1 / 3 m) C_{\text {max }}^{*} \leq 4 / 3 C_{\text {max }}^{*} .
\end{aligned}
$$

This bound was also proved to be tight.

The SPT (Shortest Processing Time) is also a list scheduling algorithm, but it orders the jobs by non-decreasing processing time. This algorithm is optimal for the problem of minimizing the sum of the completion times of the jobs $\left(\sum C_{i}\right)$, also called mean flow time and denoted by $P \| \sum C_{i}$ [BCS74].

\subsection{Multi-objective scheduling}

Some scheduling problems are too complex to be solved using only single-objective optimization. In those cases, the problem is often characterized by two or more criteria that must be taken into account simultaneously. Those criteria may reflect transverse or even conflicting interests from different entities of the problem. These problems are classified as multicriteria scheduling problems. 
Let us assume, for example, that there are two criteria $X_{1}$ and $X_{2}$ to be minimized. If $X_{1}$ is considered to be more important than $X_{2}$, a natural approach is, first, to find the optimal value of $X_{1}$, denoted by $X_{1}^{*}$, and then, in a second stage, optimize $X_{2}$ subject to the additional constraint that $X_{1} \leq(1+\eta) X_{1}^{*}$, where $\eta$ as a given threshold (possibly 0 ). This approach is called hierarchical (or lexicographic) optimization [Hoo05]. If both criteria are considered equally relevant, then a different view of simultaneous minimization refers to the concept of non-dominated - or Pareto optimal - solutions.

This concept was first used by Vilfredo Pareto, an Italian economist, in his studies of economy efficiency and income distribution. This concept captures the trade-off between two or more objectives to be optimized and can be used for comparing multi-objective solutions. In this context, a solution A is better than a solution B if B is Pareto dominated by A. Intuitively, a solution A is Pareto optimal if it is not possible to improve one of its objectives without worsening the others. Next, we borrow the definition of Pareto dominance and Pareto optimality from [Voo03] and [Hoo05], considering $X=\left\{X_{1}, X_{2}, \ldots, X_{k}\right\}$ as a set of objectives to be minimized and a pair of schedule solutions $S$ and $S^{\prime}$.

Definition 2.1. A solution $S$ Pareto dominates a solution $S^{\prime} \Leftrightarrow \forall l \in\{1, \ldots, k\}, X_{l}(S) \leq$ $X_{l}\left(S^{\prime}\right)$

and $\exists l \in\{1, \ldots, k\} \mid X_{l}(S)<X_{l}\left(S^{\prime}\right)$

Intuitively, this means that if $S$ Pareto dominates $S^{\prime}$ then values on $S$ are equal or less than $S^{\prime}$ values, being that at least one of the inequalities is strict. Solutions that are not Pareto dominated by any other solution is said to be Pareto optimal.

Definition 2.2. Schedule $S$ is Pareto optimal or non-dominated if there is no feasible schedule $S^{\prime} \neq S$ such that $S^{\prime}$ Pareto dominates $S$.

In multi-objective optimization problems, we are interested in finding solutions that are not Pareto-dominated by any other solution. This set of non-dominated solutions is called the Pareto set.

Figure 2.4 shows a visualization of a Pareto set for a multi-objective problem with two objectives, $X_{1}$ and $X_{2}$. The points $A, B, C, D, E$ and $F$ are all possible solutions to the 


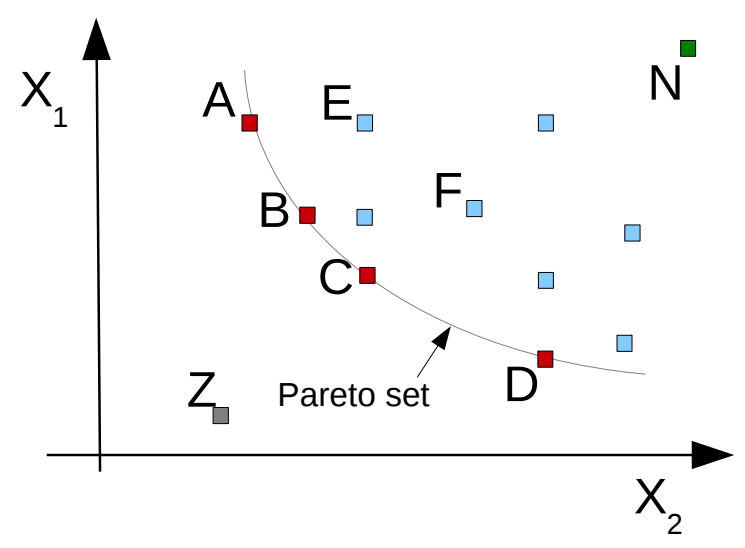

Figure 2.4: Pareto set for the set of solutions $X=\left\{X_{1}, X_{2}\right\}$

problem. The point $Z$, called Zenith, represents the best possible solution to $X_{1}$ and $X_{2}$, if they were considered individually, in a dedicated system. In contrast, the point $N$, called Nadir, represents the worst possible solution for both objectives. In this figure, the Pareto set is represented by the curved line. The points $A, B, C, D$ are Pareto optimal, while points $E$ and $F$ are Pareto dominated.

The main works related to this thesis address the problem of optimizing criteria from many users simultaneously. This problem was first studied on a single processor with two users by Agnetis et al. [AMPP04] and extended to multiple processors by Saule and Trystram [ST09].

Agnetis et al. [AMPP04] analyzed several scenarios with two users varying the objective function adopted by each one and the structure of the processing system. They were interested in Constrained Optimization Problems where one objective is fixed as a constraint while the second objective is optimized. Following the Graham classification scheme, this problem is indicated as $1 \| f^{A}: f^{B} \leq Q$ where $f^{A}$ and $f^{B}$ are the objective functions of the users and $Q$ is an integer. Formally, the problem is to find a schedule $\alpha^{*}$ such that $f^{B}\left(\alpha^{*}\right) \leq Q$, and $f^{A}\left(\alpha^{*}\right)$ is minimum. Given this, they provided $\mathrm{a}<\overline{1}, \overline{1}>$-approximation polynomial algorithm for the problem of two users interested in minimizing their makespan on a common processing resource. This notation means that for given $w=\left(w_{1}, w_{2}\right)$ thresholds for the values of the objective functions $f^{(1)}, f^{(2)}$, the algorithm delivers a solution where $f^{1} \leq 1 . w_{1}$ and $f^{2} \leq 1 . w_{2}$. 
The authors also show that when both users are interested in the sum of completion times, the problem becomes also binary NP-hard and they provide a pseudo-polynomial dynamic program to solve it. With mixed objectives, if one user is interested in the weighted sum of completion times, the problem is binary NP-hard. Other cases are polynomial.

Saule and Trystram [ST09] analyzed the Multi-Users Scheduling Problem (MUSP), namely, the problem of scheduling independent sequential jobs belonging to $k$ different users on $m$ identical processors. In this problem, each user selects an objective function among makespan and sum (weighted or not) of completion times. This is an offline problem where all the jobs are known in advance and can be immediately executed. This problem becomes strongly NP-hard as soon as one user aims at optimizing the makespan. For the case where all users are interested in the makespan, denoted by $\operatorname{MUSP}\left(k: C_{\max }\right)$, the authors showed that the problem can not be approximated with a vector ratio better than $(1,2, \ldots, k)$. This is a natural extension of the approximation ratios notation where the $u$-th number of the vector corresponds to the approximation ratio on the $u$-th user objective.

The term "no vector-ratio better than $\left(\rho_{1}, \rho_{2}, \ldots, \rho_{k}\right)$ " stands for the component wise relation, which means that the vector-ratio $\left(\rho_{1}, \ldots, \rho_{i-1}, \rho_{i}-\epsilon, \rho_{i+1}, \ldots, \rho_{k}\right)$ is not feasible. However, this formulation does not prevent a $\left(\rho_{1}, \ldots, \rho_{i}-\epsilon, \ldots, \rho_{j}+\epsilon, \ldots, \rho_{k}\right)$ vector-ratio from existing. For example, consider the vector-ratio $H=(3,3,3,3)$. If there is no vectorratio better than $H$, then the vector-ratio $(3,3,2,3)$ is not feasible while $(4,3,2,3)$ may be feasible.

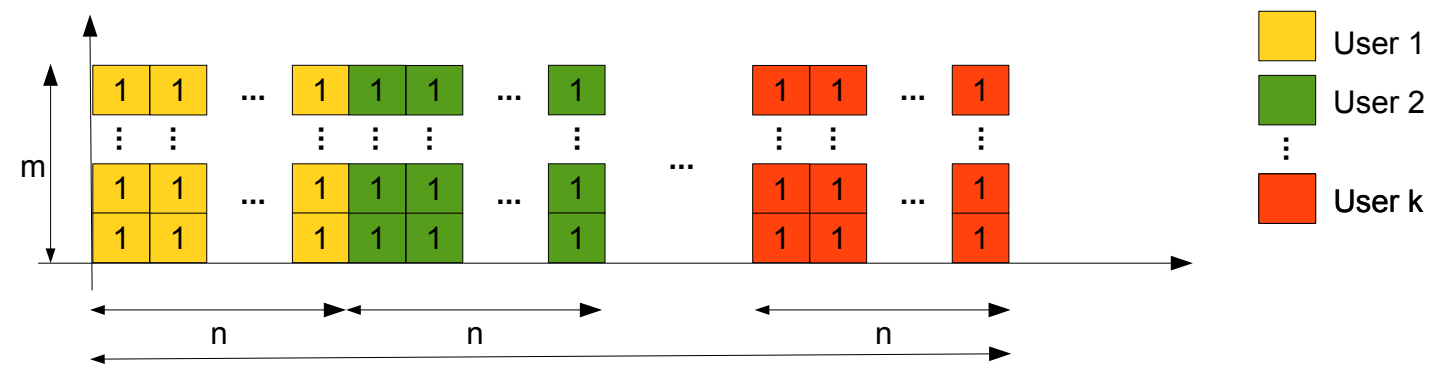

Figure 2.5: Approximability bound for MUSP

The proof stating that $\operatorname{MUSP}\left(k: C_{\max }\right)$ can not be approximated with a performance vector-ratio better than $(1,2, \ldots, k)$ considers an instance with $k$ users. In this instance, 
each user has $m . n$ jobs, where $m$ is the number of machines and $n$ is any integer. All the jobs have the same length $p=1$ and the absolute best makespan that can be achieved for each user is $n$, while in any efficient schedule one user will have a makespan of $n$, another one will have a makespan of $2 n$, and so on until the last user with a makespan of $k n$. Thus, it is impossible to obtain an algorithm that guarantees a vector-ratio better than $(1,2, \ldots, k)$. This can be easily visualized in Figure 2.5 .

Note that in an efficient schedule, the set of jobs of one user is scheduled all at once, as a single block. Each user block is followed by another user block, the resulting schedule being all the users blocks, one after the other. If we take a job scheduled at time $t_{i}$ and change its position with a job from another user scheduled at time $t_{j}>t_{i}$, we end up with a schedule whose vector-ratio is worst than $(1,2, \ldots, k)$ since two users will have $t_{j}$ as their $C_{\max }$ values. The resulted vector-ratio will be $\left(1,2, \ldots, t_{j}, t_{j}, \ldots, k\right)$, where the values are in non-decreasing order. This is analogous for the case $t_{i}>t_{j}$.

Figure 2.6 illustrates the trade-offs between the objectives of two users. As a matter of simplicity, only a single machine is used in this example. In this figure, user 1 (light gray) owns two jobs of length 4, while user 2 (dark gray) has two jobs of lengths 3 and 7. All the scheduling possibilities are presented as points on the graph, where $C_{\max }^{1}$ in the x-axis and $C_{\max }^{2}$ in the y-axis represent the $C_{\max }$ values of user 1 and user 2, respectively. Point $\mathrm{Z}$ represents the $C_{\max }$ lower bound of both users (zenith), while the upper bound (nadir) is unlimited $^{1}$. Points $A$ and $E$ are optimal in the sense that there is no better solution that improves one objective without degrading another one. Points $B$ and $F$ are derived from $A$ by exploring different positions for the first job of user 2 . But both solutions degrades the $C_{\max }$ of user 1 without improving the $C_{\max }$ of user 2 . The same applies for points $C$ and $D$ relative to $E$. Thus, according to the concept of Pareto dominance, we say that $B, C, D$, and $F$ are Pareto-dominated solutions.

Based on these observations, Saule and Trystram [ST09] proposed an algorithm for $\operatorname{MUSP}\left(k: C_{\max }\right)$ called MULTICMAX and proved that it is a $(\rho, 2 \rho, \ldots, k \rho)$-approximation. Each position of this vector ratio represents the performance ratio of one of the $k$ users

\footnotetext{
${ }^{1}$ Empty spaces between jobs can push both $C_{\max }$ values to an unlimited extent.
} 

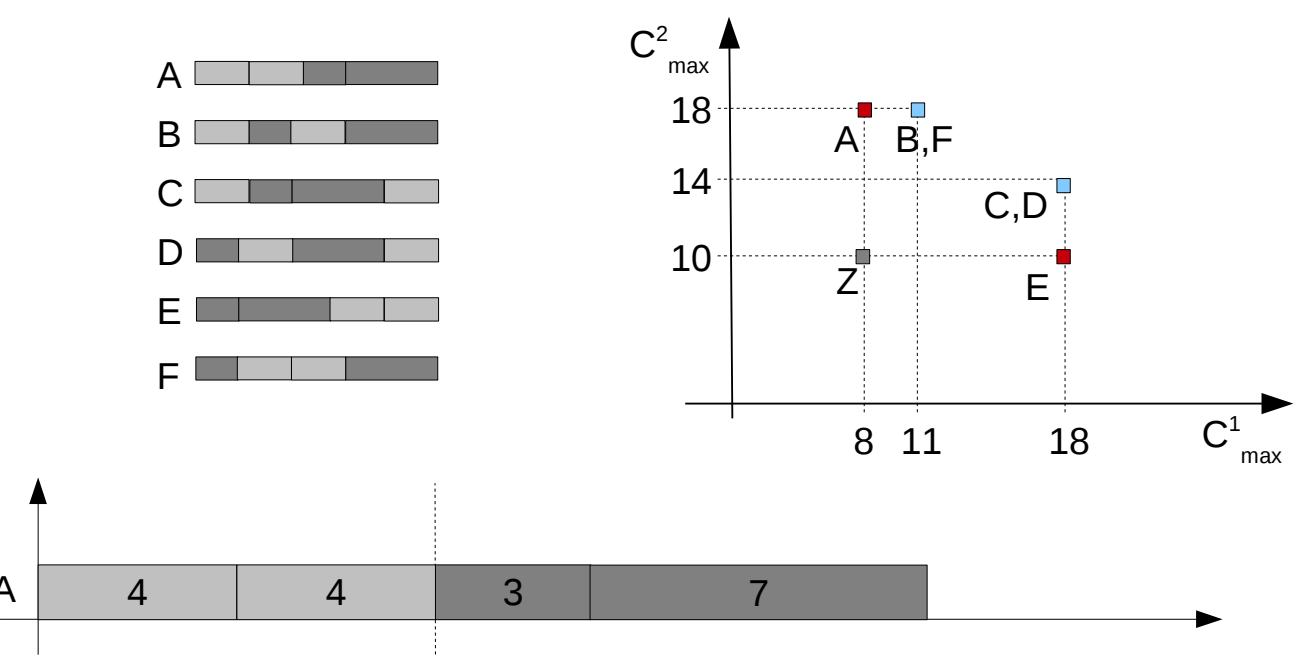

Figure 2.6: Pareto optimality for MUSP (user 1 in light gray, user 2 in dark gray)

and $\rho$ is the approximation ratio of an algorithm for the single-user case (Hochbaum and Shmoys proposed a PTAS to this problem by using dual approximation [HS87]). They also proposed an algorithm (called MULTISUM) for the case where all users are interested in the sum of completion times and proved that it is a $(k, \ldots, k)$-approximation.

The algorithm MULTICMAX works as follows: for each user $u$, it computes a schedule $S^{u}$ with a $\rho$-approximation algorithm. Then, it sorts the users by non-decreasing values of $C_{\max }^{u}\left(S^{u}\right)$, where $C_{\max }^{u}\left(S^{u}\right)$ denotes the $C_{\max }$ value of schedule $S^{u}$. Finally, it schedules the jobs of user $u$ according to $S^{u}$ between $\Sigma_{u^{\prime}<u} C_{\max }^{u^{\prime}}\left(S^{u^{\prime}}\right)$ and $\Sigma_{u^{\prime} \leq u} C_{\max }^{u^{\prime}}\left(S^{u^{\prime}}\right)$. Examining again the example of Figure 2.6, the solution $A$ would be the one generated by this algorithm.

The theorem and the proof stating that MULTICMAX is a $(\rho, 2 \rho, \ldots, k \rho)$-approximation of $\operatorname{MUSP}\left(k: C_{\max }\right)$ can be seen in [ST09]. This theorem is valid for a given unknown permutation of users as one user can not know in advance his/her rank in the algorithm. The vector-ratios are computed relatively to an absolute best solution which is usually unfeasible, but the authors emphasize that this is reasonable since it ensures the performance degradation of each user.

Indeed, this algorithm generates a final schedule that might contains idle spaces. Those may appear between two consecutive blocks of jobs from different users, allowing many portions of the system to remain unusable. As an example, consider an instance with 
two users, each of them with $\lfloor m / 2\rfloor$ jobs of length $p$ and $m>1$ (number of machines). MULTICMAX generates a final schedule of length $2 p$ using $\lfloor m / 2\rfloor$ machines, while the optimal schedule is of length $p$, with each user occupying one half of the machines. But this is a situation where the absolute best makespan for both users is feasible, which is not the general case. So, still in this work, the authors presented a class of solutions where each user submits a reasonable number of jobs that follows a linear function on the number of machines. They showed that this class of solutions are MULTICMAX with $\rho=2$ and that it contains efficient schedules that are close to the Pareto set.

\subsection{Fairness in scheduling}

Fairness is an important issue while designing scheduling policies and it has gained growing attention from computer scientists in the last decade [SKS04, SS05, RLAI04, VC05, $\mathrm{IPC}^{+}$09, CM10, Pro13, KPS13]. However, it is still a fuzzy concept that has been handled in many different ways, varying according to the target problems. It is said that resources are fairly shared if they are equally available to the parties, or are available in proportion to some criterion (e.g. money income, user hierarchy, etc.) [Dro09]. The definition of available and equity, however, are subject to interpretation.

For example, if the resource is shared by three users, namely A, B and C, and they are assigned shares $\mathrm{x}, \mathrm{y}$ and $\mathrm{z}$, then their jobs will receive fractions $\frac{x}{x+y+z}, \frac{y}{x+y+z}, \frac{z}{x+y+z}$, respectively. For a resource to be equally available, it can be determined that all the parties have an equal share of the resource at any time moment. In this example, this would be $x=y=z=1 / 3$.

There are two classical approaches to share a resource in a system: space sharing and time sharing. In space sharing, the resource is divided into subsets that are assigned to each party. This can be more easily applied to divisible resources such as computer memory, bandwidth and parallel systems. For indivisible resources like single processing units and I/O devices, time sharing may be a more appropriate approach, since it gives time slices to the parties in a round-robin fashion. During each time slice the resource is available to just one user. 
Figures 2.7 and 2.8 are examples of space-sharing and time-sharing schedules. In these examples, a parallel machine with $m$ processors is shared by 3 users identified by different shades of gray and each user has the same share of the processors.

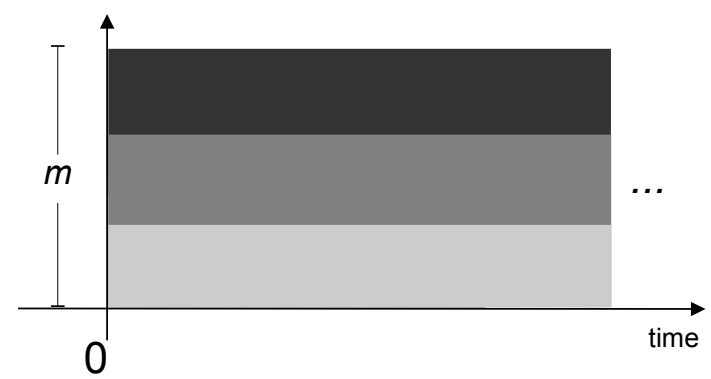

Figure 2.7: Space-sharing schedule example with 3 users

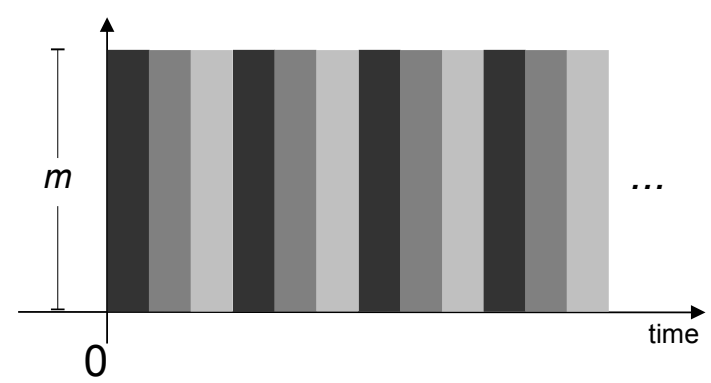

Figure 2.8: Time-sharing schedule example with 3 users

Users' satisfaction or wishes (i.e. utilities), however, is a function of not only the assigned resources, but also the needs. If the needs are unequal, even if the resources are allocated according to the assigned shares, the resulting utilities will differ.

For instance, consider a system with $m$ processors shared by two users $u$ and $v$. User $u$ submits a sequential job of 10 hours of processing time while the user $v$ submits $m$ jobs of one hour each. Assume the jobs are non-preemptive.

Using a time-sharing algorithm, one user will be executed after the other in their respective time slices. In this case, the time slice must be equal or greater than 10 hours. Otherwise, the job of $u$ would never get executed. But even so, executing one user after the other in distinct time slices will produce different utilities since one user needed a processing time 10 times larger than the other and waiting 10 additional hours has different impacts for each user. 
In turn, using a space-sharing algorithm implies in giving to users $u$ and $v$ half of the processors. However, it is clear that giving half of the processors to user $u$, that needed only one processor, results in a different utility than giving the other half to the user $v$, that needed all the resources to speed up the execution of his/her jobs.

Moreover, sharing of resources according to space-share policy may be Pareto-inefficient. Consider another example with two users submitting $m$ jobs of one hour each, with each user being given half of the processors. In this case, both users will wait 2 hours. If instead all the processors would be given to one user and then to the other, the completion time of the first user would be improved, while the completion time of the second user would remain the same.

This example is depicted in Figure 2.9 where $C_{\max }^{1}$ is the makespan of dark gray user and $C_{\max }^{2}$ is the makespan of light gray user. The space-share solution represented by $B$ is Pareto dominated by the time-share solution represented by $A$.

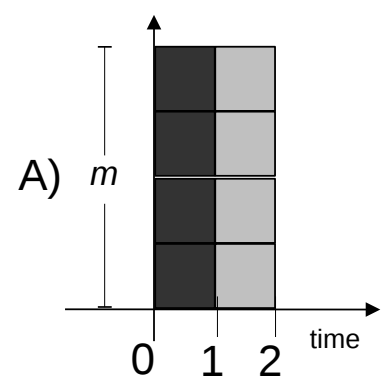

Time Share

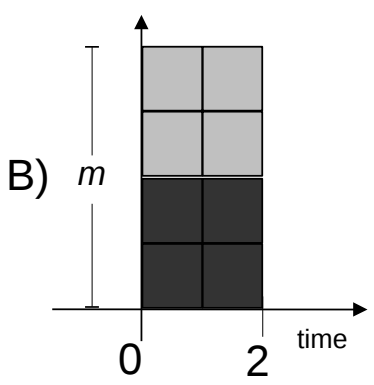

Space

Share

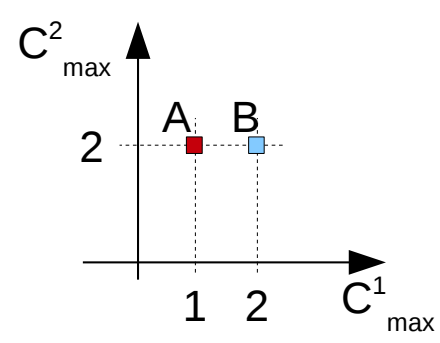

Pareto solutions

Figure 2.9: Examples of space-share and time-share schedules with 2 users and corresponding Pareto solutions

In fact, neither strict space-sharing nor strict time-sharing can solely produce reasonable fair schedules in parallel systems. In this thesis it is shown how this can be achieved through a combination of both strategies, embedded with a fair allocation policy. But first, it has to be defined how fairness and utilities are formally measured.

Throughput-oriented users, for example, are interested in maximizing the job throughput, i.e. number of jobs executed per time unit. So, maximize the minimum throughput is 
the correct measure for having a greater number of satisfied users. In turn, response-time oriented users - the type of interest in this work - are concerned about minimizing the job response time (also called flow time), i.e. the time their jobs spent in the system. Likewise, minimize the maximum flow-time (i.e. max-flow-time) seems to be the right measure. However, flow time solely is not appropriate, because giving the same flow time for all jobs results in worst performances for short jobs, compared to the ones obtained by long jobs. So, in order to do a correct comparison, the job lengths must be taken into account. The stretch metric is the one used to comply this.

The stretch is defined as the flow time normalized by the job's processing time. More formally, considering a job $\mathrm{J}$, the stretch of $\mathrm{J}$ is:

$\frac{\text { J's completion time - J's submission time }}{J \text { 's processing time }}$.

The stretch and flow metrics were first studied by Bender et al. [BCM98] for continuous job streams. Stretch optimization was also studied for independent tasks without preemption [BMR02], Bag-of-Tasks applications [LSV06, CM10], multiple parallel task graphs [CDS10] and for sharing broadcast bandwidth between clients requests [WC01].

A job stretch measures how the performance of a job is degraded compared to a system dedicated exclusively to this job. Thus, the stretch measures the relative responsiveness of the system and quantifies the user expectation that the flow time should be proportional to the imposed load. For example, it may be fine for a 2 hours job to be executed within 3 hours since its submission (resulting in a stretch of 1.5). However, for a 30 minutes job this delay may be unacceptable (it would result in a stretch of 6).

As an analogy with the concept of distributive justice presented in the introduction, two jobs $\mathrm{J} 1$ and $\mathrm{J} 2$ are equally treated if they have the same stretch. That is:

$\frac{\text { J1's completion time - J1's submission time }}{\text { J1's processing time }}=\frac{\text { J2's completion time - J2's submission time }}{\text { J2's processing time }}$.

The completion time is the job "reward", that is, what is obtained from the scheduler. In this case, the lower, the better. The submission time represents the job "cost" in terms of scheduling effort: the sooner a job was submitted, less effort is needed from the scheduler to deliver the expected "reward". Finally, the processing time is the job "investment" in 
an inversely proportional relation: short processing times represents more investments to achieve better rewards than long processing times.

In this thesis, the stretch metric is adapted to the notion of job campaigns. This is detailed in Chapter 3.

In order to optimize stretch, it is worth to analyze some strategies. Unfortunately, common approaches such as First-Come-First-Served (FCFS) and classical list scheduling strategies are not well-adapted. FCFS is maybe the simplest and still more largely used scheduling algorithm. It executes jobs according to a FIFO order (First In, First Out), that is, in the order that they arrive in the system. Other well known scheduling policies as LPT [Gra69] (Longest Processing Time first), SPT [BCS74] (Shortest Processing Time first) and their derivatives focus on job lengths to achieve single objective optimization such as overall makespan or throughput. The execution priority is given individually to the jobs according to their lengths. In LPT, longer jobs have bigger priorities while in SPT, shorter jobs are prioritized.

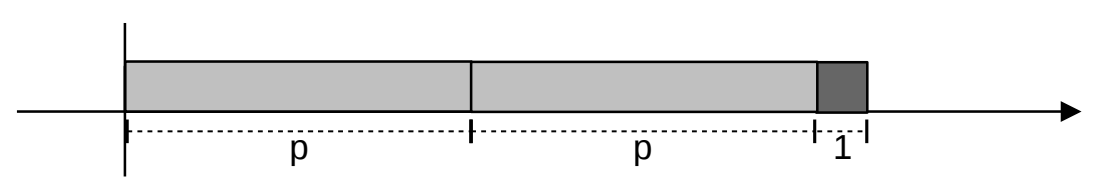

Figure 2.10: FCFS worst-case ratio with 2 users

Figures 2.10, 2.11 and 2.12 illustrate examples of schedules constructed by FCFS, LPT, and SPT algorithms. In these figures, two users, identified by two shades of gray, submit their parallel jobs of length 1 and $p$ on a single machine. All the jobs are ready to execute from the beginning of the schedule. It is well-known that these policies do not embrace user related information such as user identity and submission frequency. Hence, they do not grasp the sense of justice in a multi-user environment. But, even if they did, these policies could bring very bad stretch experiences to users.

FCFS, for instance, can be unfair to users who always submit small jobs. One can easily realize that a small job can wait an arbitrarily long time to start since the system is fully occupied with the execution of jobs submitted earlier by another users. Assuming, for 
example, that both users start submitting jobs at time 0 and that each job of a user is submitted as soon as the previous finishes, one user can be systematically delayed by the other. This is depicted in Figure 2.10 where the resulting stretch for the dark gray user is far from the optimum by a factor of $2 p$.

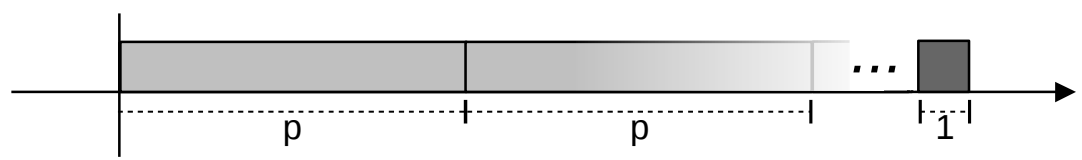

Figure 2.11: LPT starvation with 2 users

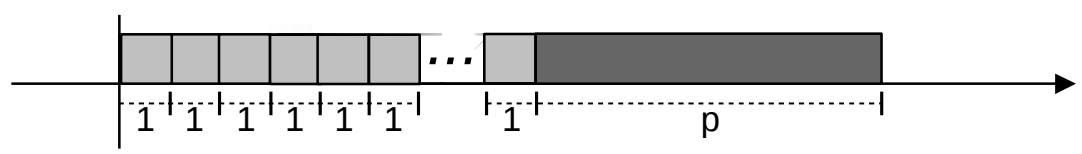

Figure 2.12: SPT starvation with 2 users

In turn, policies whose ordering is based on job length like SPT and LPT are subject to job starvation if applied without a dynamic priority mechanism. These are the cases depicted in figures 2.11 and 2.12. In both figures, new arriving jobs from the light gray user can indefinitely delay jobs from the dark gray user. In those cases, the resulting stretch for the dark gray user can be arbitrarily far from the optimum.

When it comes to policies and mechanisms implemented on actual systems like PBS [Hen95], OAR [CDCG ${ }^{+}$05] and Slurm [YJG03], most of them supports multilevel queue scheduling and backfilling. Multilevel queue scheduling is a powerful mechanism on which jobs are organized into different queues according to some classification criterion. Each queue is given a distinct priority and the jobs are FIFO ordered and executed. It is often used to separate different types of jobs (e.g. batch, interactive, etc.) or to reflect the user hierarchy of the system (e.g. administrator jobs could be placed on a different queue than user jobs). So, regarding fairness between users, priority queues are as fair as hierarchical systems can be, if used solely.

Backfilling can be used to fill the idle gaps between jobs and increase system utilization. 
It consists in searching for idle spaces backwards in the schedule in which upcoming jobs can be placed. This can be done in a more or less aggressive way regarding the delay of previously scheduled jobs. This technique does not deliver individual guarantees to users regarding performance neither equitable treatment [SKSS02], however, it is a powerful mechanism that, in practice, offers significant scheduler performance improvement.

In [RLAI04] and [SKS04], several metrics are proposed for expressing the degree of unfairness in various systems. Both works evaluate the unfairness of algorithms such as FCFS, backfilling and processor sharing, but fairness is associated with the jobs and their service requirements. Thus, the concept of fairness is always taken from a job point-of-view as "fairness between jobs" instead of "fairness between users" as it is supported in this thesis.

In the real world, fair-share derived mechanisms are implemented by some parallel system schedulers. Some examples are the Hadoop Fair Scheduler [Zah], the fair-share policy of Maui Scheduler [JSC01] and the Fair-share Factor of Slurm Multifactor Priority Plugin [YJG03, Geo10].

In Hadoop, fairness is obtained by space-sharing. It divides the resources among distinct pools, each one belonging to a user. Figure 2.13 depicts a space-share schedule with the 6 resources being equally divided among 3 pools. At the beginning, $M_{1}, M_{2}$, and $M_{3}$ belong to the pool of dark gray user, while $M_{4}, M_{5}$, and $M_{6}$ belong to the pool of medium gray user. At time $t=2$ the light gray user joins the system. Now, $M_{1}$ and $M_{2}$ form the dark gray pool, $M_{3}$ and $M_{4}$ form the light gray pool, and $M_{5}$ and $M_{6}$ form the medium gray pool. The pools are also changed in $t=8$ and $t=12$ as users leave the system. In Hadoop, weights can be applied to the pools, giving more or less priority to them, but in this example all pools have the same weight.

Observe that, using space sharing, the stretch experienced for each user is linearly proportional to the number of users. All the user workloads are equally stretched and, thus, the schedule is fair.

However, this solution has some drawbacks. First, the jobs are assumed to be malleable. But when scheduling rigid or moldable jobs, the final schedule would be hardly seamless 


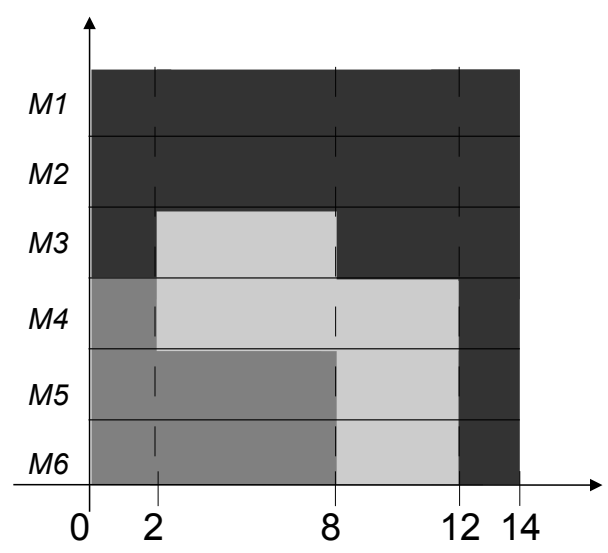

Figure 2.13: Example of Hadoop Fair Scheduler pools with 3 users

and without gaps as the one showed on the figure. Second, the division between resources and users needs to be an integer, otherwise, one or more machines would need to be shared using time-sharing. Third, this solution gives bad stretches for all users and is likely Pareto-dominated by another solution. As stated early on space-share schedules, it would be more interesting for some users to obtain better stretches while not changing the stretch of others.

The Maui Scheduler treats fairness among users as a secondary objective. A "negative karma" (i.e. penalty that reduces the priority of a job) can be assigned to users submitting many tasks and so, users who submit less frequently can be prioritized.

Similarly, the fair-share factor of Slurm gives the order of execution of a job based on the share of the resources allocated to the job's user and the resources the user has already consumed. Those jobs whose users are under-serviced are scheduled first, while jobs whose users are over-serviced are scheduled when the machine would otherwise go idle.

Note that this characterizes a time-sharing algorithm as all the resources are used by one user at a time. However, instead of giving time slices for each user, a user can use the resources for as long as he/she remains with the higher priority.

The Fair-share Factor $F$ is calculated periodically for each user according to the following simplified formula:

$$
F=2^{-U_{\Delta t} / S}
$$


where $S$ is the normalized share and $U_{\Delta t}$ is the normalized usage factoring in half-life decay.

In a system with $k$ users and no user hierarchy, the normalized share $S=1 / k$ and the normalized usage is calculated as:

$$
U_{\Delta t}=U_{u s e r(\Delta t)} / U_{t o t a l}(\Delta t)
$$

where $U_{u s e r(\Delta t)}$ is how much time the jobs of the user consumed from the processors over a fixed time period $\Delta t$ and $U_{\text {total }(\Delta t)}$ is the total consumption from all the jobs over that same time period.

So, the factor $F$ is a value between zero and one, where one represents the highest priority and zero the lowest. A factor of 0.5 indicates that the user has used exactly the portion of the machine that was allocated to him/her, above 0.5 it indicates that the user has consumed less than the allocated share, and below 0.5 it indicates that the user has consumed more than the allocated share. The user factors are recalculated periodically according to a predefined time interval $\Delta t$ and the user with the highest factor value has the highest priority.

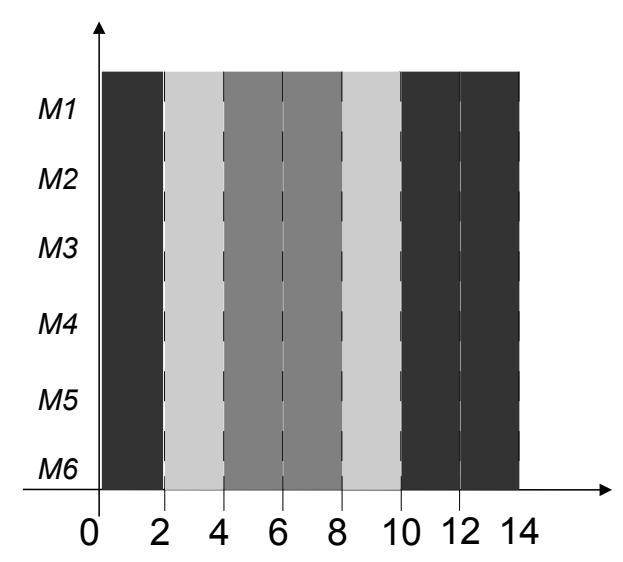

Figure 2.14: Example of Slurm schedule with 3 users using Fair-share factor and $\Delta t=2$

Figure 2.14 depicts a time-share schedule from Slurm in which the factors are recalculated every 2 minutes $(\Delta t=2)$. The order on which the users join and leave the system is the same as in the Hadoop example. At $t=0$, only the dark gray and medium gray users are present and their factors are $F=0.5$. As both have the same priority, the dark user 
is randomly chosen and until $t=2$ only dark gray jobs are executed. At $t=2$ the factors are recalculated. Also, the light gray user joins the system. Now, the dark gray user has $F=0.125$ while the other users have $F=1$. As light and medium gray users have the same priority, the light gray user is randomly chosen and until $t=4$ only light gray jobs are executed. This process is repeated at every time interval until all jobs get executed. Note that from $t=10$ there is only dark gray jobs to be executed and so there is only dark gray jobs until the end.

This solution has some advantages over the Hadoop Fair Scheduler. First, each user gets all the resources, so the problem of dividing the resources evenly between users is no longer present. Second, it is more responsive: the stretches for each user workload were equal or better than the ones in the space-share schedule of Hadoop.

However, this solution has also some drawbacks. Similar to Hadoop, the final schedule would be hardly seamless and without gaps when scheduling rigid or moldable jobs. Further, users get bad factors for using the resources even when there is no other user present on the system. Ideally, no user should be punished for making use of resources that would be idle otherwise [Dro09]. 


\section{Chapter 3}

\section{The Campaign Scheduling Problem}

Computing infrastructures for parallel processing are generally multi-user, requiring that users, alone or grouped in projects, share the same environment to perform theirs jobs. In HPC clusters, for example, several processors are grouped to empower the execution of CPU intensive applications from diverse research fields as particle physics, weather prediction, seismic data processing, and bio-informatics.

The management of processors is commonly done by a Resource Management System (RMS) whose main part is the job scheduler. Typically, jobs are submitted in batch and placed on one or several queue(s) before being executed.

In a typical shared parallel system, each user performs many submissions over time driven by scientific questions related to the project he/she belongs to. Some users may perform many submissions with few jobs in a short period of time, while others may perform few submissions with many long jobs in a very sparse way. In short, daily scientific activities such as exploratory analysis, simulations, fine-tuning, system prototyping, algorithm comparison, and benchmarks are commonly performed several times interleaved by idle time periods during which the users may analyze previous results or prepare their next submissions. This user activities towards a common goal can be seen as a many-step interactive workflow where each step is called a "campaign".

In this thesis, we fit this notion of campaign in what we called the campaign model. 


\subsection{The campaign model}

A campaign is composed of jobs and the job scheduler is responsible for organize the campaigns, ordering their execution according to a specific policy. As soon the execution of a campaign is finished the campaign owner is able to submit his/her next campaign. It is clear that, from the user point of view, the sooner his/her jobs are executed, the better. So, the objective of each user is to minimize the time each campaign spent in the system. It starts with the submission and it ends when all the jobs are executed. Namely, this is the campaign's flow time.

To give an example, Figure 3.1 illustrates a campaign submission from a user in a system with 4 machines (named $M_{1}, M_{2}, M_{3}$ and $M_{4}$ ). The medium gray rectangles represent jobs from the user while the light gray rectangles represent jobs from other users. Note that, at the time of the submission, all the machines are occupied so the campaign does not start to be executed immediately.

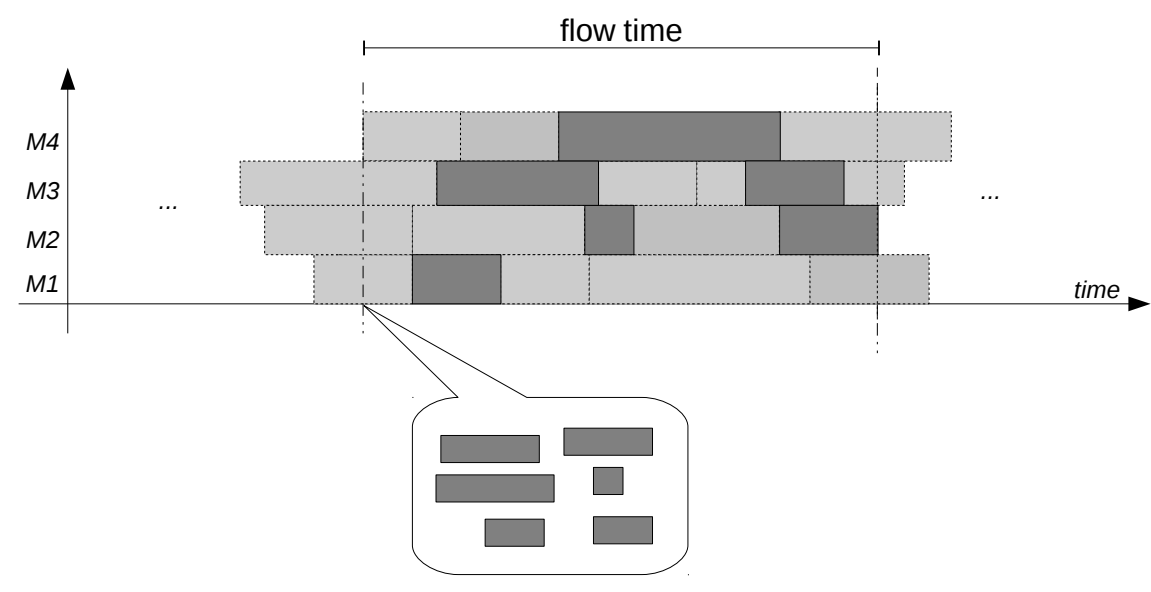

Figure 3.1: Campaign submission and execution on a 4 processor system

\subsection{Related models}

In this section, we present some examples of real application models and systems, and how they are related to campaign scheduling. 


\subsubsection{Bag-of-Tasks}

Bag-of-Tasks (BoT) is an application model composed of tasks to be executed independently from each other. The tasks can be executed in any order and there is almost no communication between them. There are only two points of communication: at the beginning, for passing the initial parameters for each task, and in the end, for transmitting the results.

The parallelization of these applications is so trivial that they are also known as embarrassingly parallel applications. Some examples of areas that can make use of the BoT model are data mining, Monte Carlo simulations, distributed rendering, and particle physics. This model is often used in parallel system with communication constraints and desktop based platforms like BOINC [And04].

\subsubsection{Parameter sweep applications.}

Another common example is found in parameter sweep applications. These applications scan a wide range of possible parameter values and iteratively "zooms" into promising areas. The computation is performed in stages: (i) testing multiple values of parameters; (ii) choosing the most promising area and generating parameter values for the next iteration. Stages are repeated until the quality of the obtained solution does not improve significantly in subsequent iterations.

The summary step is easy to compute. It is performed either off-cluster, or in a centralized node. The most computationally-expensive step is the testing step. The application can be modeled as a set of independent jobs $\left\{T_{i}\right\}_{i=1, \ldots, n}$. Each job is a set of files and a file might be the input to one or more jobs. Each job tests a certain combination of parameters. Jobs are moldable; they scale almost linearly up to a certain number of processors. The runtime of jobs can be estimated.

\subsubsection{BSP model.}

The BSP is a programming model introduced by Leslie Valiant that combines software and architecture [Val90]. Processing applications written using the BSP paradigm 
is a twofold procedure: the program is a sequence of super-steps and each super-step comprises three phases, namely computation, communication and global synchronization. In the computation phase, each machine executes one sequential job. The computations are independent in the sense that they execute asynchronously from all others. In the communication phase, the jobs communicate between themselves to exchange data. When a job has no more data to exchange, it enters in the synchronization phase where it waits until all the jobs finish their communications.

One of the advantages of this model is its simplified interface, which facilitates the implementation of BSP libraries and parallel applications based on the BSP model. Another advantage is the performance predictability that can be analyzed assuming that one time unit in one processor can perform one operation in a data available in its local memory.

But this model has also some disadvantages, as the need of periodic synchronizations among the processes. This become very costly as the cluster becomes larger. Also, depending of how frequent these synchronizations happen, the performance can be compromised.

\subsubsection{Similarities and applicability}

The examples presented on the previous sections share some characteristics with the campaign model. In all of them each user shares the resources with others and performs many submissions that composes the user workload. Terminologies like batch, stage, phase, and step are interchangeable as they share the same concept of campaigns. Another point in common is that applications fitting these models are more likely to be response-time based than throughput based. When a campaign is submitted, users want to gather the partial results as quickly as possible in order to prepare the subsequent campaign. But, despite these similarities, there are some particular differences regarding mainly the job model, the time interval between campaigns, and the level of clairvoyance.

These models, for example, do not specify job preemptiveness. This is defined by the model implementation on a particular system. Most BOINC projects, for instance, implement the bag-of-task model using preemptive jobs. This means that the scheduler can interrupt jobs of one user without loss of computation. Campaigns from two or more users 
can even be split in several parts and interleaved, without idle spaces between them.

For the BSP, there is one straightforward approach to map this model into campaign scheduling: each superstep can be seen as a single campaign. The length of each phase is particular to each job and it depends on the machine that was allocated to it. But the synchronization is a barrier that symbolizes the end of a campaign. As no campaign can start before the end of the previous one, no superstep can start until the previous superstep has ended. Two or more BSP applications competing for the same set of resources can be expressed as a multi-user campaign scheduling problem. It is up to the scheduler to decide the most honest way of sharing the resources among the applications.

Despite of the fork-join shape of these examples, the campaign model described in this thesis can be applied to many situations since it models an execution pattern: submissions of independent jobs from many users over time. Gathering partial results and elaborating the next input can automatically be executed by one of the tasks like is done in BSP. In this case, all the tasks are processed without idle times between campaigns. However, this is not the general case.

In summary, human intervention between campaigns may take place or not. It depends on the type of application and/or interaction with the system. In some cases, the user can choose when the next submission will be released and, therefore, the time interval between the campaigns can be arbitrary.

\subsection{Definitions and notation}

The model consists of $k$ users (indexed by $u$ ) sharing the resources on a parallel platform composed of $m$ identical processors managed by a centralized scheduler. Figure 3.2 illustrates some of the used notation.

A user workload is composed of successive campaigns where each campaign (indexed by $i$ ) is submitted at a time denoted by $t_{i}^{u}$ and is composed of a set of independent and non-preemptive jobs.

A campaign is defined as the set $J_{i}^{u}$ containing the jobs released by a user $u$ in one submission; $n_{i}^{u}$ denotes the number of jobs of a campaign and $n^{u}$ the total number of jobs 


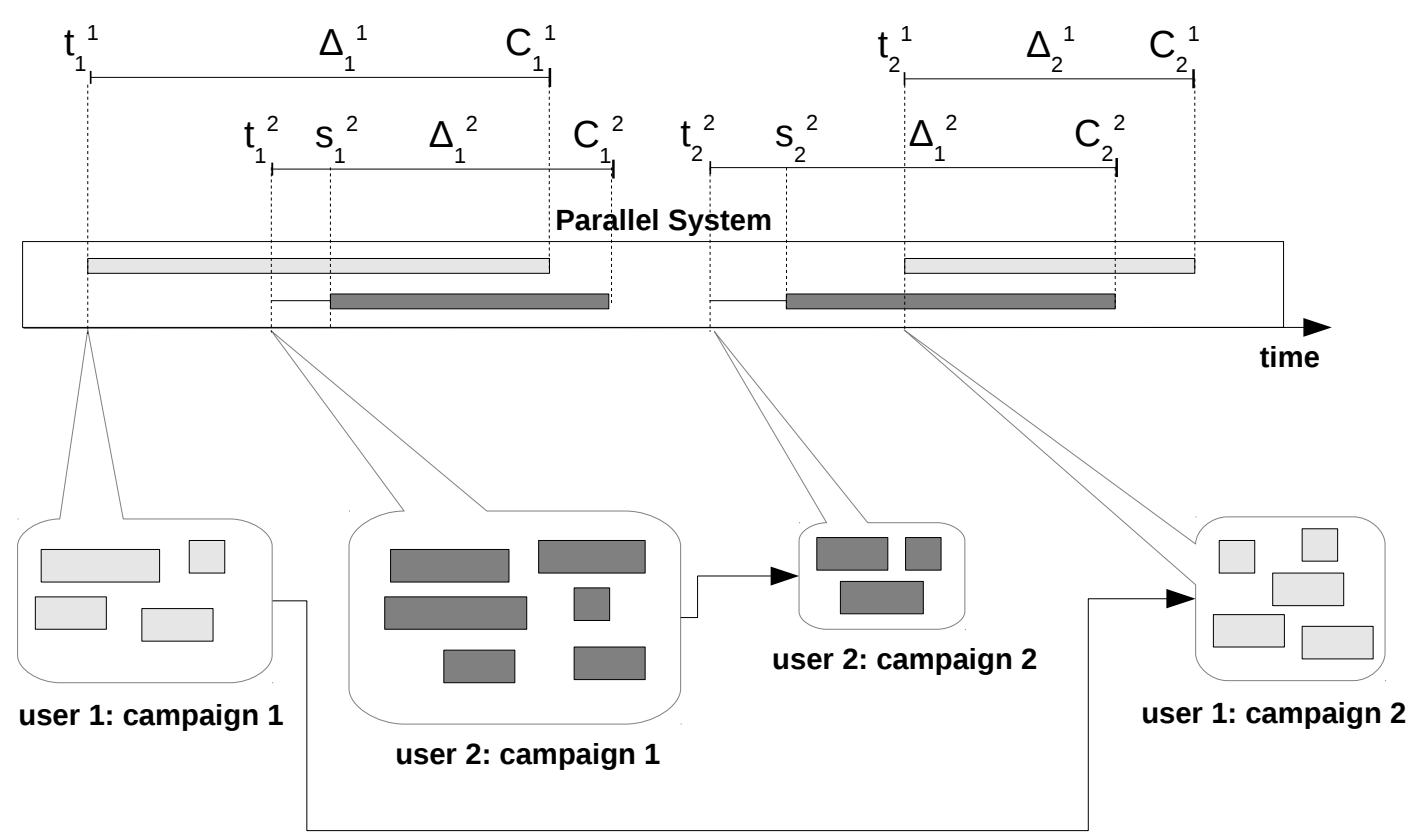

Figure 3.2: Campaign Scheduling with 2 users (user 1 in light gray, user 2 in dark gray)

released in all the campaigns of user $u$. The jobs inside a campaign are indexed by $j$, so $J_{i, j}^{u}$ denotes job $j$ from campaign $J_{i}^{u}$.

The job's length is denoted by $p_{i, j}^{u}$ and the job's size is denoted by $q_{i, j}^{u}$. So, the total workload within campaign $i$ is: $W_{i}^{u}=\sum_{j} p_{i, j}^{u} \cdot q_{i, j}^{u}$. But, when size is not mentioned, jobs should be considered sequential. In these cases, $W_{i}^{u}=\sum_{j} p_{i, j}^{u}$. This is assumed in most of this thesis, with the exception of Chapter 6.

A job, once started, cannot be interrupted nor preempted. The job start time is denoted by $s_{i, j}^{u}$ and its completion time is denoted by $C_{i, j}^{u}$.

The start time of a campaign $i$ of user $u$ is denoted by $s_{i}^{u}$. It is defined as the time the first job starts, so $s_{i}^{u} \triangleq \min _{j} s_{i, j}^{u}$. The campaign's completion time $C_{i}^{u}$ is the completion time of the last job to finish its execution: $C_{i}^{u} \triangleq \max _{j} C_{i, j}^{u}$.

The campaign's flow time, denoted as $\Delta_{i}^{u}$, is equal to the amount of time the jobs of a campaign stay in the system: $\Delta_{i}^{u} \triangleq C_{i}^{u}-t_{i}^{u}$.

The campaign's stretch is denoted by $D_{i}^{u}$ and is defined as a natural generalization of a job's stretch. Formally, the stretch of job $i, D_{i, j}^{u}$, is equal to the relative degradation of its flow time, $D_{i, j}^{u}=\left(C_{i, j}^{u}-t_{i, j}^{u}\right) / p_{i, j}^{u}$, where $p_{i, j}^{u}$ is the job length (and, thus, the job's 
Table 3.1: Notation summary

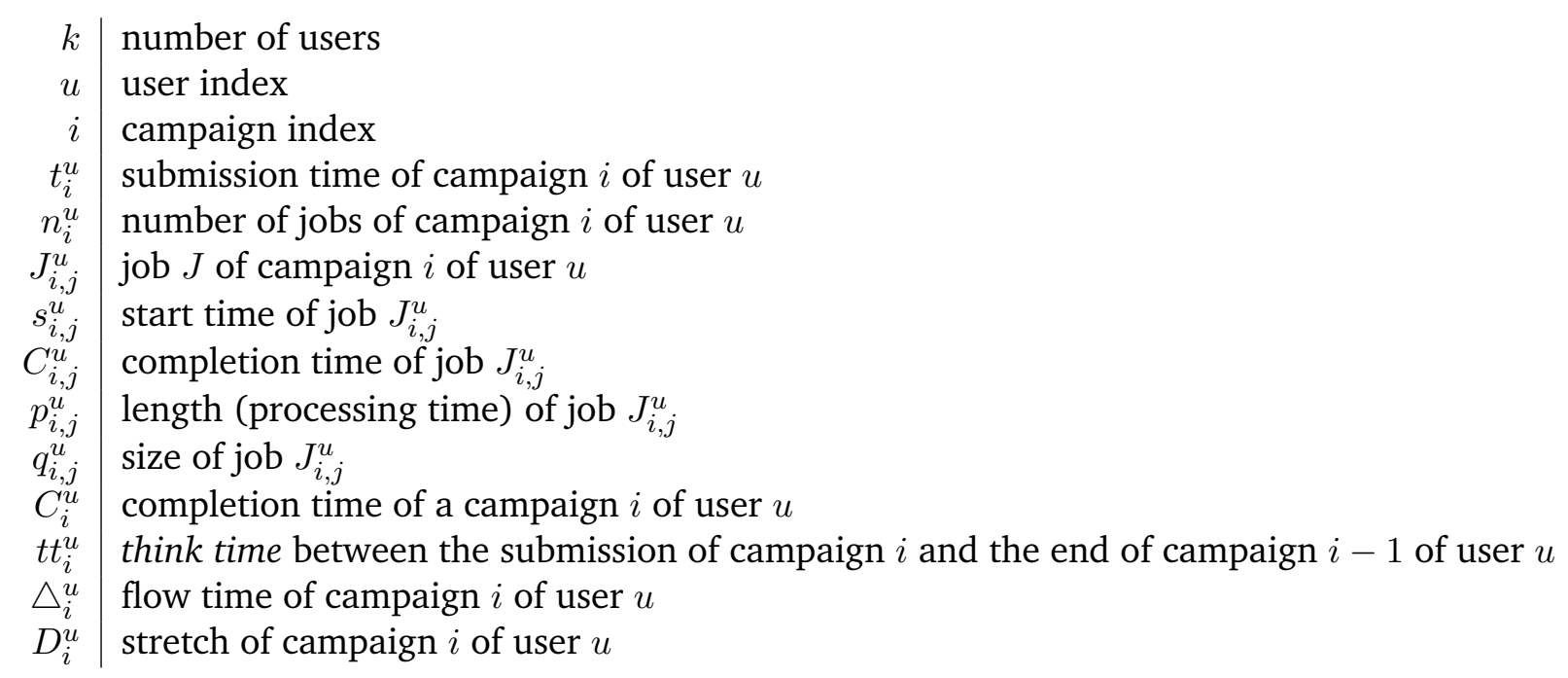

optimum flow time) [BCM98]. Determining a single campaign's optimum flow time means solving the general scheduling problem, which is NP-hard. Thus, instead, we use a lower bound on campaign's flow time defined by $l_{i}^{u}=\max \left(W_{i}^{u} / m, p_{\max }^{u}\right)$, where $p_{\max }^{u}=\max _{j} p_{i, j}^{u}$. Consequently, the campaign's stretch is defined as $D_{i}^{u}=\Delta_{i}^{u} / l_{i}^{u}$.

User $u$ cannot submit her/his next campaign $i+1$ until her/his previous campaign $i$ completes, thus $t_{i+1}^{u} \geq C_{i}^{u}$. The time between the completion of campaign $i$ and the submission of the next one $(i+1)$, called the think time, is denoted as $t t_{i+1}^{u}=t_{i+1}^{u}-C_{i}^{u}$.

The notation is summarized in Table 3.1.

From an individual point of view, each user $u$ wants to minimize the sum of campaigns' lengths $\Sigma \Delta_{i}^{u}=\Sigma_{i}\left(C_{i}^{u}-t_{i}^{u}\right)$. This objective is justified by the interactive behavior of users when submitting tasks on a cluster: each user is interested in quickly obtaining the results of individual batches of jobs rather then the job throughput in a time frame.

Using a natural extension of the classical three-field notation $\alpha|\beta| \gamma$ introduced by Graham, Lawler, Lenstra and Rinnooy Kan [GLLRK79], the resulting problem can be denoted by $P|\operatorname{camp}| \sum \Delta^{u}$.

However, from the perspective of collective justice, the objective (of the system scheduler) is to minimize the per-user and per-campaign stretch $D_{i}^{u}$. We consider stretch as it weights the responsiveness of the system by the assigned load; it is natural to expect that small workloads will be computed faster than larger ones. We consider the stretch on a 
per-user and per-campaign basis, as this results in fairness of the system towards individual users. Moreover, optimizing the stretch of each campaign (rather than the overall stretch) guarantees that not only the final result, but also partial ones, are timely computed. In this case, the resulting problem can be denoted by $P|\operatorname{camp}| \max \left\{D_{i}^{u}\right\}$.

The problem of minimizing per-user and per-campaign stretch $D_{i}^{u}$ is NP-hard. Even when restricted to a single user $(k=1)$ and to a single campaign, it is equivalent to the classical problem of minimization of the makespan on identical parallel processors $\left(P \| C_{\max }\right)$ [ST09, PRT12].

In the offline version of the problem, all the campaigns are known in advance (the number and the lengths of the jobs) and each campaign can be submitted immediately after the completion time of the previous one. In the online problem, each campaign (including its submission time) is unknown until it is submitted. From this point, the length and duration of each job is available.

The offline model may seem with no applicability at first, since to predict the number of campaigns and their respective job lengths is unrealistic. But this model is well-suited for some types of applications, like clairvoyant fork-join applications. In this type of application, each set of fork-type tasks can be mapped onto campaigns and each join task represents a single-job campaign. Furthermore, the offline set can always be used as a reference to analyze the competitiveness of online algorithms ${ }^{1}$.

In this thesis, both models are analyzed and solutions are given to them.

\subsection{Offline scheduling of single user's campaigns}

In this section, we analyze an offline version of the multi-campaign problem restricted to a single user. This problem constitutes an absolute lower bound for the multi-user case, since no objective value can be lower for a user than the one achieved when she/he is alone in the system. We denote this problem by $P|\operatorname{camp}| \sum \Delta$ (i.e. without the superscribed $\mathrm{u}$ ).

We show below that the problem is NP-hard. Then, we show that the optimal makespan

\footnotetext{
${ }^{1}$ Competitive analysis is a method for analyzing online algorithms. In this method, the performance of an online algorithm is compared to the performance of an optimal offline algorithm in which all the future job submissions are known in advance.
} 
of $P|c a m p| \sum \Delta$ with $\sigma$ campaigns is at most $\sigma$ times longer than the optimal makespan of an instance with the same jobs, but no campaigns (i.e. the same as $P \| C_{\max }$ in which all the jobs are ready to execute from the beginning of the schedule). Finally, we show that a $\rho$-approximation algorithm for $P \| C_{\max }$ is also a $\rho$-approximation for $P|\operatorname{camp}| \sum \Delta$.

Proposition 3.1. $P|c a m p| \sum \Delta$ is NP-hard. The boundary problem is $P 2|c a m p| C_{\max }$. The processing times are arbitrary.

Proof. (straightforward) The proof is by reduction from the two-processor scheduling problem $P 2 \| C_{\max }$. An instance of $P 2|| C_{\max }$ can be converted to an instance of $P 2|c a m p| C$ by placing all the jobs in the same campaign $\left(J_{j} \rightarrow J_{1, j}\right)$.

For the subsequent analysis of this section, we use the concept of campaign-compact schedule, which is defined as follows:

Definition 3.1. A campaign-compact schedule $\varsigma_{c c}$ (also called a non-delay schedule) is a schedule in which jobs from a subsequent campaign start immediately after the completion of the previous campaign, i.e., $\forall i \in\{1, \ldots, \sigma-1\} \exists j: s_{(i+1), j}=C_{i}$.

In an offline model, it is enough to consider only campaign-compact schedules because, for a single user, the class of campaign-compact schedules represent all "reasonable" schedules.

A schedule that is not campaign-compact can be transformed to a campaign-compact schedule by shifting the campaign submission times to coincide with the completion of their previous ones. This transformation reduces completion times of some jobs and the length of the whole schedule. Thus, the optimal schedule is also campaign-compact. In the remainder of this section, a schedule is always considered to be campaign-compact.

The following lemma binds the completion time $C_{i}$ of a campaign with the durations of the previous campaigns; moreover, it shows that $C_{\sigma}=\sum \Delta$.

Lemma 3.1. In a schedule, the completion time of the $i$-th campaign $C_{i}$ is equal to the sum of durations of the previous campaigns and the current campaign: $\forall_{i} C_{i}=\sum_{i^{\prime}=1}^{i} \Delta_{i^{\prime}}$ 
Proof. Follows directly from the definition of $\Delta_{i}$ and the Definition 3.1.

The notion of a campaign restricts the set of feasible schedules; thus the optimal schedule for the campaign problem $P|c a m p| \sum \Delta$ might be longer than the optimal schedule for a similar instance with all the jobs in a single campaign.

Proposition 3.2. An optimal schedule for an instance of the problem $P|c a m p| \sum \Delta$ with $\sigma$ campaigns is at most $\sigma$ times longer than the optimal schedule for an instance of $P \| C_{\max }$ with the same jobs, that is: $C_{\sigma}^{*} \leq \sigma C_{\max }^{*}$, where $C_{\sigma}^{*}$ denotes the optimal completion time of the $\sigma$-th campaign and $C_{\max }^{*}$ denotes the optimal $C_{\max }$ for $P \| C_{\max }$. This bound is tight.

Proof. First, from Lemma 3.1, $C_{\sigma}^{*}=\sum_{i=1}^{\sigma} \Delta_{i}^{*}$, where $\Delta_{i}^{*}$ is the duration of the $i$-th campaign in the optimal schedule. Second, given that each campaign is a subset of jobs, $\Delta_{i}^{*} \leq C_{\max }^{*}$. Thus, $\sum_{i=1}^{\sigma} \Delta_{i}^{*} \leq \sigma C_{\max }^{*}$.

For the tightness of the bound, consider an instance with $m$ jobs of unitary length (remember that $m$ is the number of processors). An optimal schedule with a single campaign schedules all the jobs in parallel, with the completion time of $C_{\max }=1$. If all the jobs belong to different campaigns $(\sigma=n)$, they must be executed sequentially, thus $\sum_{i=1}^{\sigma} \Delta_{i}^{*}=\sigma$.

Consequently, spreading the jobs in multiple campaigns "costs" the system at most $\sigma$ (the total number of campaigns).

Another interesting question is what is the impact of using a $\rho$-approximation algorithm to schedule the jobs inside a campaign. The following result states that any $\rho$-approximation algorithm for the standard job scheduling problem can be used to obtain a $\rho$-approximation for the campaign problem.

Proposition 3.3. Any scheduling algorithm $A$ that is a $\rho$-approximation for $P \| C_{\max }$ is also a $\rho$-approximation for $P|c a m p| \sum \Delta$. The algorithm for $P|c a m p| \sum \Delta$ (denoted by A-camp) creates a schedule by, first, performing $\sigma$ executions of $A$, that is, executing A separately for each campaign; then shifting the schedule of the $i$-th campaign by $C_{i-1}$.

Proof. From Lemma 3.1, $C_{\sigma}=\sum_{i} \Delta_{i}$. As the algorithm used to schedule each campaign is a $\rho$-approximation, $\Delta_{i} \leq \rho \Delta_{i}^{*}$. Thus, $C_{\sigma} \leq \rho \sum_{i} \Delta_{i}^{*}$, and as $C_{\sigma}^{*}=\sum_{i} \Delta_{i}^{*}, C_{\sigma} \leq \rho C_{\sigma}^{*}$. 
In summary, the barrier between subsequent campaigns affects the system by increasing the makespan at most $\sigma$ times. Yet, the resulting Campaign Scheduling problem can use the same algorithm as the similar standard scheduling problem to obtain the same approximation ratio.

\subsection{Offline scheduling with multiple users}

In this section, we study the offline version of the Campaign Scheduling problem with multiple users, denoted by $P|\operatorname{camp}| \sum \Delta^{u}$. In this scenario, the campaigns are clairvoyant (i.e. all the jobs from all the campaigns are known in advance) and each user submits all the campaigns at once to be scheduled in sequence. Even with this restriction, this problem is NP-hard as the boundary problem with one user $\left(P|c a m p| \sum \Delta\right)$ is NP-hard (see Section 3.4).

First, it is shown that this problem cannot obtain a better approximation vector-ratio than $(1,2, \ldots, k)^{2}$. Then, we apply a multi-campaign version of the MULTICMAX algorithm to obtain a $(\rho, 2 \rho, \ldots, k \rho)$-approximation solution. Finally, we reason about the tradeoff between fairness and performance on this solution and why it is not sufficiently fair.

Proposition 3.4. $P|c a m p| \sum \Delta^{u}$ can not be approximated with a performance vector-ratio better than $(1,2, \ldots, k)$.

Proof. Let us consider the following instance. All campaigns of all $k$ users have $m$ jobs of unitary length, where $m$ is the number of machines. All users have the same number $n$ of campaigns. Obviously, for each user independently, the best $\sum_{i} \Delta_{i}^{u}$ achievable is equal to $n$. But, in any efficient schedule, one user will have $\sum_{i} \Delta_{i}^{u}$ equal to $n$, another one will have $\sum_{i} \Delta_{i}^{u}$ of $2 n$ and so on. Thus, it is impossible to guarantee a vector-ratio better than $(1,2, \ldots, k)$.

Remark that any permutation of this vector-ratio can be obtained (as explained in Section 2.3). But this vector-ratio is not accomplished by all schedules. Consider for example a schedule that interleaves campaigns of users similarly to the round-robin scheduling.

\footnotetext{
${ }^{2}$ The meaning of term "no vector-ratio better than.." is recalled from Section 2.3
} 
Each $\Delta_{i}^{u}$ will be of length 1 . So, in this schedule, one user will have $\sum \Delta_{i}^{u}=k n$, another one will have $k n-1$, and so on until $k n-k$. Clearly, the corresponding vector-ratio will be worse than $(1,2, \ldots, k)$.

Based on this observation, we propose the MULTICAMP algorithm. This is an algorithm for the offline problem inspired on the MULTICMAX algorithm [ST09] as it schedules users' campaigns in blocks.

First, for each campaign $i$ of each user $u$, the algorithm computes a schedule $\varsigma_{i}^{u}$. Usually, $\varsigma_{i}^{u}$ will be distant from the optimal by a factor. We denote this approximation factor as $\rho$. Then, for each user, the algorithm puts all its campaigns' schedules side by side to form a single block, i.e. a campaign-compact schedule $\varsigma^{u}$ (see Definition 3.1). The length of this schedule $\varsigma^{u}$ is $\sum_{i} \Delta_{i}^{u}$. Next, the users are sorted by non-decreasing values of $\sum_{i} \Delta_{i}^{u}$. Finally, the algorithm places the block $\varsigma^{u}$ of user $u$ between $\sum_{u^{\prime}<u} \sum_{i} \Delta_{i}^{\left(u^{\prime}\right)}$ and $\sum_{u^{\prime} \leq u} \sum_{i} \Delta_{i}^{\left(u^{\prime}\right)}$.

Proposition 3.5. MULTICAMP is a $(\rho, 2 \rho, \ldots, k \rho)$-approximation algorithm of $P|c a m p| \sum \Delta^{u}$.

Proof. First, we have to verify the validity of the final schedule. In fact, the campaign's blocks are scheduled in disjoint intervals of length $\sum_{i} \Delta_{i}^{u}\left(\varsigma^{u}\right)$ according to $\varsigma^{u}$. Second, we verify that the final schedule has a performance vector-ratio of $(\rho, 2 \rho, \ldots, k \rho)$. The users are ordered by increasing values of $\sum_{i} \Delta_{i}^{u}\left(S_{i}^{u}\right)$ and each block is scheduled in sequence according to this order. Thus, $\sum_{i} \Delta_{i}^{u} \leq u \sum \Delta_{i}^{u}\left(S_{i}^{u}\right)$. Moreover, $\varsigma_{i}^{u}$ was generated by a $\rho$-approximation algorithm. Thus, $\sum_{i} \Delta_{i}^{u} \leq u \sum \rho \Delta_{i}^{u *}=u \rho \sum \Delta_{i}^{u *}$.

Usually, a performance vector-ratio depending on $k$, a parameter of an instance, is not considered as an efficient solution. However, the optimal value of each particular objective function is obtained by scheduling the jobs of the user as if she/he was the only user on the system. The performance vector-ratio represents the distance between the schedule given by our algorithm and these absolutely optimal solutions for every user (the zenith point). In the general case, the zenith solution is not feasible.

In MULTICAMP, although the response time of an entire block of campaigns of a user (i.e. the total user workload) is bounded, individual campaigns are arbitrarily delayed. User $u$ may be disappointed that he/she has to wait $u-1$ workloads to be processed before 
his/her own workload starts to be executed. It should be more fair to the users if everybody was equally affected or proportionally affected according to their own workload lengths.

In Section 2.3 we saw that, considering just one campaign (as in the MUSP problem), is not a good idea to interleave jobs as it leads to inefficient, Pareto dominated solutions. However, Pareto efficient solutions inherit an imbalance between users with respect to the response time, given that campaigns are ordered according to their lengths. With multiple campaigns, we can reduce this imbalance by giving higher priority in the next scheduling decisions for the users that were "unhappy" in the previous ones. We tackle this problem in the next section.

\subsection{Online scheduling with multiple users}

The offline solution is not suitable for the online version of the problem because campaigns are unknown until they are submitted. The scheduler has no knowledge about the user's will to submit more campaigns than the ones that were already submitted, so it is impossible to sort and prioritize users according to the total workload lengths.

An online algorithm should consider both user fairness and performance. As we do not know the lengths of the workloads in advance, both criteria should be respected at any time.

In this section, we first discuss how the stretch metric must be taken into account in order to provide a fair mechanism in an online fashion. Then we analyze the performance of the FCFS algorithm as it is the most used and well known online algorithm. We show that FCFS is not suitable for fair scheduling in the way we defined since it can produce an arbitrary large stretch for campaigns.

This section also serves as a preamble of the next chapter where more sophisticated online solutions are proposed.

\subsubsection{Measuring fairness by max-stretch}

A scheduling algorithm is unfair if it gives preference to the applications which use resources in a certain way, like preferring short running applications over long running 
ones [SS05]. In this sense, FCFS can be considered fair if "equity" between applications is the only think that is at stake since it treats all the applications in the same way, considering only the arrival time to order their executions.

Nevertheless, FCFS may lead to long wait times and does not take into account that different applications may belong to different users. In practice, some users may be inadvertently preferred. Yet, an algorithm considered "fair" for some context (and for some definition of "fairness") may not hold its properties in other contexts.

As each user is interested in the sum of campaigns' response times, we tackle the problem of fairness by measuring the campaign stretch. Recall that, for campaigns, the stretch of a campaign is defined as the time the campaign stays in the system normalized by the campaign's lower bound on the processing time.

To guarantee fairness between users it is necessary to choose an aggregation function. Three of them are normally considered, corresponding to the standard norms $L_{\infty}, L_{1}, L_{2}$ : minimizing maximum of stretches, sum of stretches, and product of stretches. From Section 2.3, recall that improving the objective of a user in a Pareto-optimal solution implies worsening the objective of other users. So, we consider that a Pareto optimal solution that gives the same stretch for all the campaigns is more fair than a Pareto optimal solution that gives different stretch values, even if the second produces a lower stretch sum.

According to this, a fair scheduling algorithm should strive to minimize the max-stretch because this has the side effect of producing close stretch values for all users. Thus, minimizing the max-stretch $\left(\max _{u} D^{u}\right)$ is the natural choice.

\subsubsection{FCFS in multi-user systems}

FCFS is one of the most commonly used policies in job scheduling and this can be justified not only by its simplicity but also by other surprising characteristics. As an example, FCFS is optimal for optimizing the max-flow of a set of jobs and does not require preemption nor job clairvoyance to achieve that [BCM98].

However, FCFS is not a "reactive" scheduling algorithm. A new submitted job is forced to wait the execution of all the jobs previously submitted. Intuitively, it would be more 


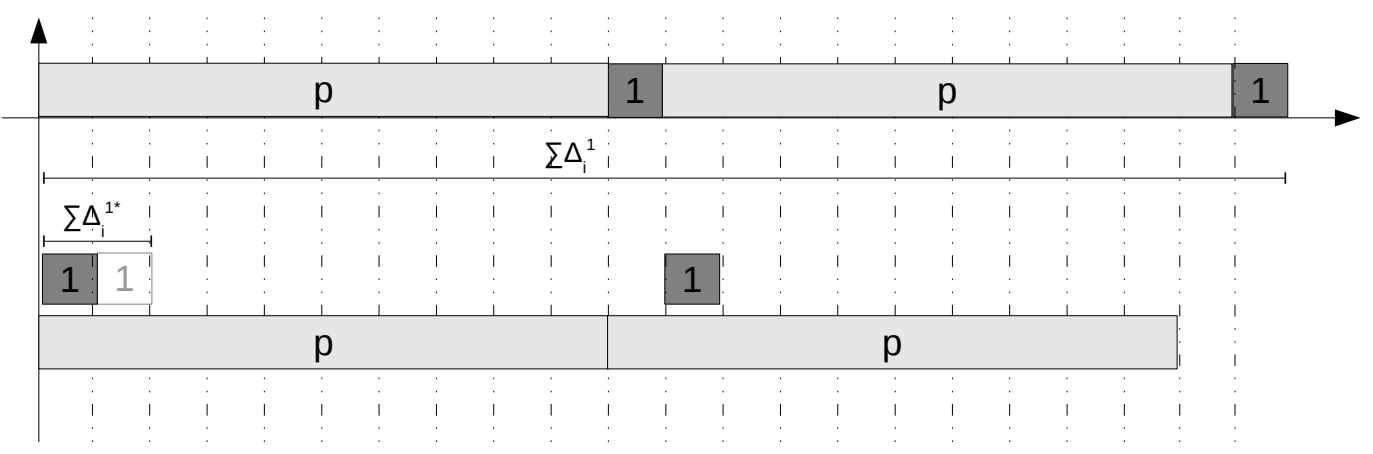

Figure 3.3: FCFS competitiveness. $k=2, \sigma=2$, user 1 in light gray, user 2 in dark gray. Max-stretch is $(2 p+2) / 2 \approx p$ (for large $p$ ); the optimal max-stretch is $(2 p+2) /(2 p) \approx 1$ (for large $p$ ). The faded campaign represents the optimal position of the second campaign for user 2.

clever to sacrifice some jobs to get something more "reactive" and also more "fair". In fact, considering a multi-user context, FCFS is arbitrarily bad for fairness.

In FCFS, the jobs are scheduled as soon as they arrive; the start time depends on the availability of the machines at the time of the submission. When a user $u$ submits a campaign $i$ at a moment $t_{i}^{u}$, it will be scheduled after all the previous submissions sent before $t_{i}^{u}$. This means that the jobs from user $u$ may start its execution in a moment $t_{i}^{u}+\varepsilon$, where the length of $\varepsilon$ is not bounded. The following proposition formally states this result (see Figure 3.3).

Proposition 3.6. FCFS is at least $\alpha$-approximation algorithm for $P|c a m p| \sum \Delta^{u}$ and for the $P|c a m p| \max \left\{D_{i}^{u}\right\}$ where $\alpha$ is the ratio between the biggest and the shortest workload length.

Proof. Consider $m$ processors and $k=2$ users. Consider that both users have $\sigma$ submissions to be made and each submission is composed of $m$ jobs. Jobs of user 1 are of length $p$ and jobs of user 2 are of length 1 . Now, consider the following situation: user 1 makes his/her first submission at time 0 and the user 2 makes his/her first submission immediately after the first user in a time $\varepsilon \approx 0$. As the jobs will be executed following a FIFO order, initially all the machines are occupied with the jobs from user 1 . User 2 will have to wait a time $p-\varepsilon$ before his/her tasks get scheduled. While the optimal schedule for the first campaign of user 2 is of length 1 , the schedule given by FCFS is of length $p+1-\varepsilon \approx p+1$. Consequently, if this submission order is repeated in the subsequent campaigns, the schedule given by 
FCFS is of length $\approx(p+1) \sigma$ while the optimal sum of campaigns' completion times ( $\left.\sum \Delta^{u}\right)$ is of length $\sigma$. Thus, we can not give any guarantees to the user 2 for the sum of campaigns' lengths based solely on an approximation from his/her particular optimal schedule.

Consequently, in the online problem, FCFS is an arbitrary bad strategy for fairness based on stretch.

In the next three chapters, we introduce new online solutions for fairness in multi-user campaign scheduling. These solutions establishes fairness between users regarding how much of their workloads is ready to be executed and how much was executed in the past. Both solutions are based on deadlines that are derived from the campaigns' workloads and from the number of users competing for the resources. 


\section{Chapter 4}

\section{Fair online schedules: constrained}

\section{scenario}

In order to maintain fairness among processes, a standard operating system commonly uses a round-robin (RR) strategy with preemption. Each process is given the processor for a fixed quantum of time and when the time expires, the process is preempted and put at the end of the queue. This strategy results in reasonable fairness among processes, as each process is slowed down proportionally to the total number of processes being executed.

Nevertheless, rather than fairness, the main objective of operating systems is CPU utilization, which is achieved by cleverly mixing I/O bound and CPU bound processes. But in parallel systems and particularly in HPC systems, CPU bound processes (also called CPU intensive) are more likely to be found. Besides, fairness is a far more important issue since HPC systems can be shared by dozens or hundreds of users. Another relevant difference is that preemption is not much used in practice $\left[\mathrm{KSS}^{+} 05\right]$.

Given these discrepancies, in this chapter we introduce an algorithm called FairCamp. This algorithm promotes fairness for a constrained scenario of Campaign Scheduling in a way similar to round-robin preemptive schedules. In this scenario the number of users $k$ is considered to be fixed and there are no time intervals between the end of a campaign and the submission of the next one, i.e., think-time is always zero or, more formally, $\forall u \forall i, t t_{i}^{u}=0$. 


\subsection{The FairCamp online algorithm}

FairCamp is a deadline based algorithm. For each submitted campaign a deadline (denoted by $d_{i}^{u}$ ) is fixed and used to order the campaigns execution. The campaign deadline is a function of the campaign's length, the number of users and the previous campaign deadline. Formally,

$$
d_{i}^{u}=k \cdot \Delta_{i}^{u}\left(\varsigma_{i}^{u}\right)+d_{i-1}^{u},
$$

where $\Delta_{i}^{u}\left(\varsigma_{i}^{u}\right)$ is the length of the campaign on the schedule $\varsigma_{i}^{u}$ generated by a $\rho$-approximation algorithm using all the processors.

In other terms, the deadline is a cumulative and recursive function. It is proportional to the number of users $k$ and shifted by the deadline of the previous campaign $d_{i-1}^{u}$.

This deadline formula is inspired on the max-stretch value for a user. From section 3.5, when analyzing MULTICAMP, it is known that $k \rho$ is the worst max-stretch a user can obtain. So, if FairCamp is able to construct a schedule where all the deadlines are not violated, it guarantees that no user is worse-off than if the resources were shared between the users with a Round-Robin, preemptive scheduler.

When a user submits a campaign, we do not know if this campaign will be the last one or if there will be more campaigns to be submitted. Thus, setting a deadline for each campaign is a way to ensure that the max-stretch will be respected for all the user submissions.

\subsection{Example}
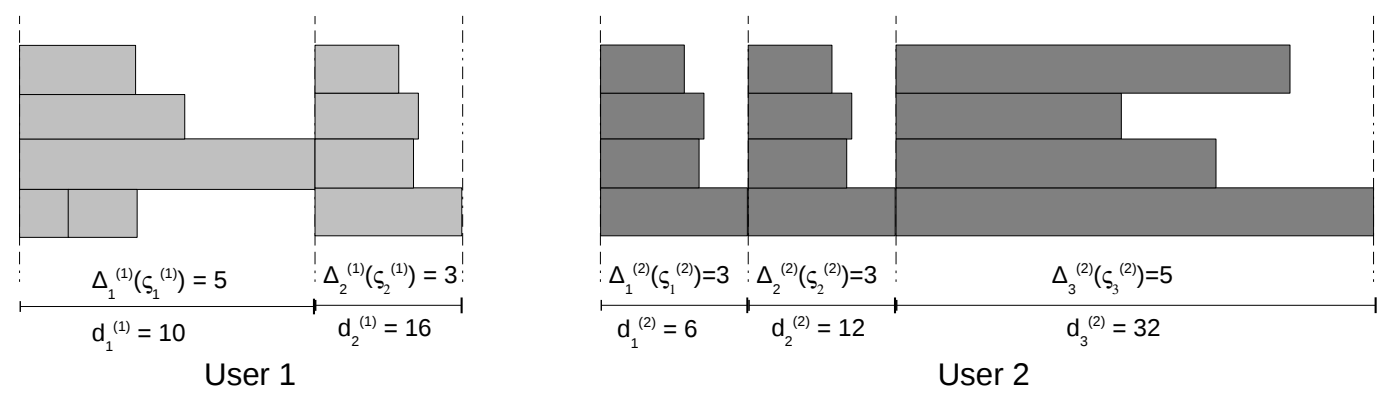

Figure 4.1: Deadlines with $k=2$ and multiple campaigns

Consider a scenario with $k=2$ users, where user 1 has $\sigma^{1}=2$ campaigns of calculated 
length $\Delta_{1}^{1}\left(\varsigma_{1}^{1}\right)=5$ and $\Delta_{2}^{1}\left(\varsigma_{2}^{1}\right)=3$. According to FairCamp their respective deadlines are $d_{1}^{1}=2 \cdot 5=10$ and $d_{2}^{1}=2 \cdot 3+10=16$. Similarly, if user 2 has $\sigma^{2}=3$ campaigns of calculated length $\Delta_{1}^{2}\left(\varsigma_{1}^{2}\right)=3, \Delta_{2}^{2}\left(\varsigma_{2}^{2}\right)=3$ and $\Delta_{3}^{2}\left(\varsigma_{3}^{2}\right)=10$, their deadlines are $d_{1}^{2}=6$, $d_{2}^{2}=12$, and $d_{3}^{2}=32$. This example is presented in Figure 4.1

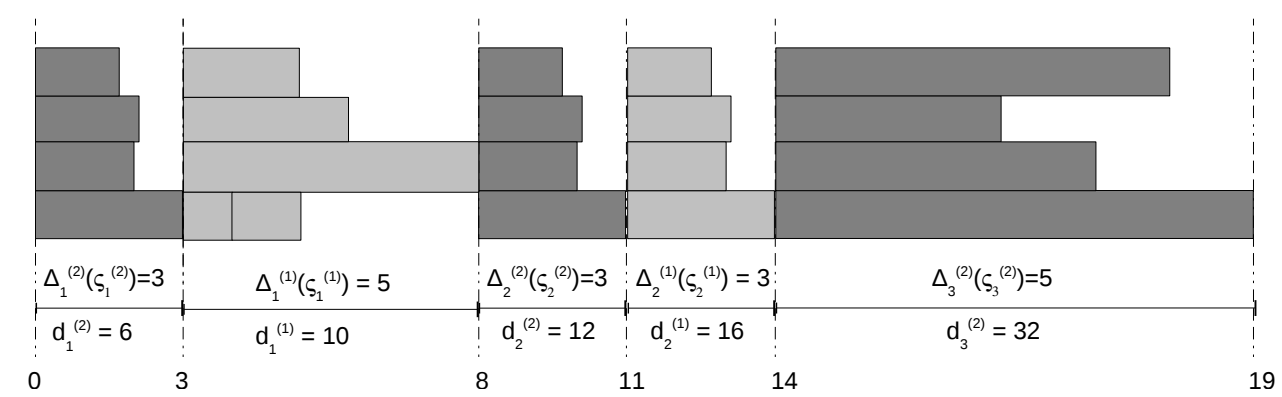

Figure 4.2: Example of schedule constructed by FairCamp (user 1 in light grey and user 2 in dark grey)

Figure 4.2 represents the schedule output. Note that the campaigns are executed according to the Earliest Deadline First order (EDF) and that all the campaigns finish before their respective deadlines.

\subsection{Algorithm description}

FairCamp uses EDF order to choose the campaigns to be executed. FairCamp is composed of two modules (Algorithm 1 and Algorithm 2) that run in parallel. They share a queue in order to place the campaigns that are ready to execute. The first module generates information about arriving campaigns and puts them into a queue. The second module chooses campaigns by EDF and schedule them as soon as the resources are available.

In line 4 of the Algorithm 1, the process stops and waits some user $u$ to release a new campaign $i$. Next, in line 5, we generate a schedule for $i$ and the resources list based on a polynomial $\rho$-approximation algorithm. Then the campaign deadline is calculated from the number of users $k$, the schedule length $\Delta_{i}^{u}$ and the deadline of the previous user campaign (line 7 and 8). Finally, a tuple containing the schedule and its deadline is generated and added to the queue.

The scheduling module performs EDF over the submitted campaigns.

Algorithm 2 iterates over the tuples on the queue searching which one has the schedule 

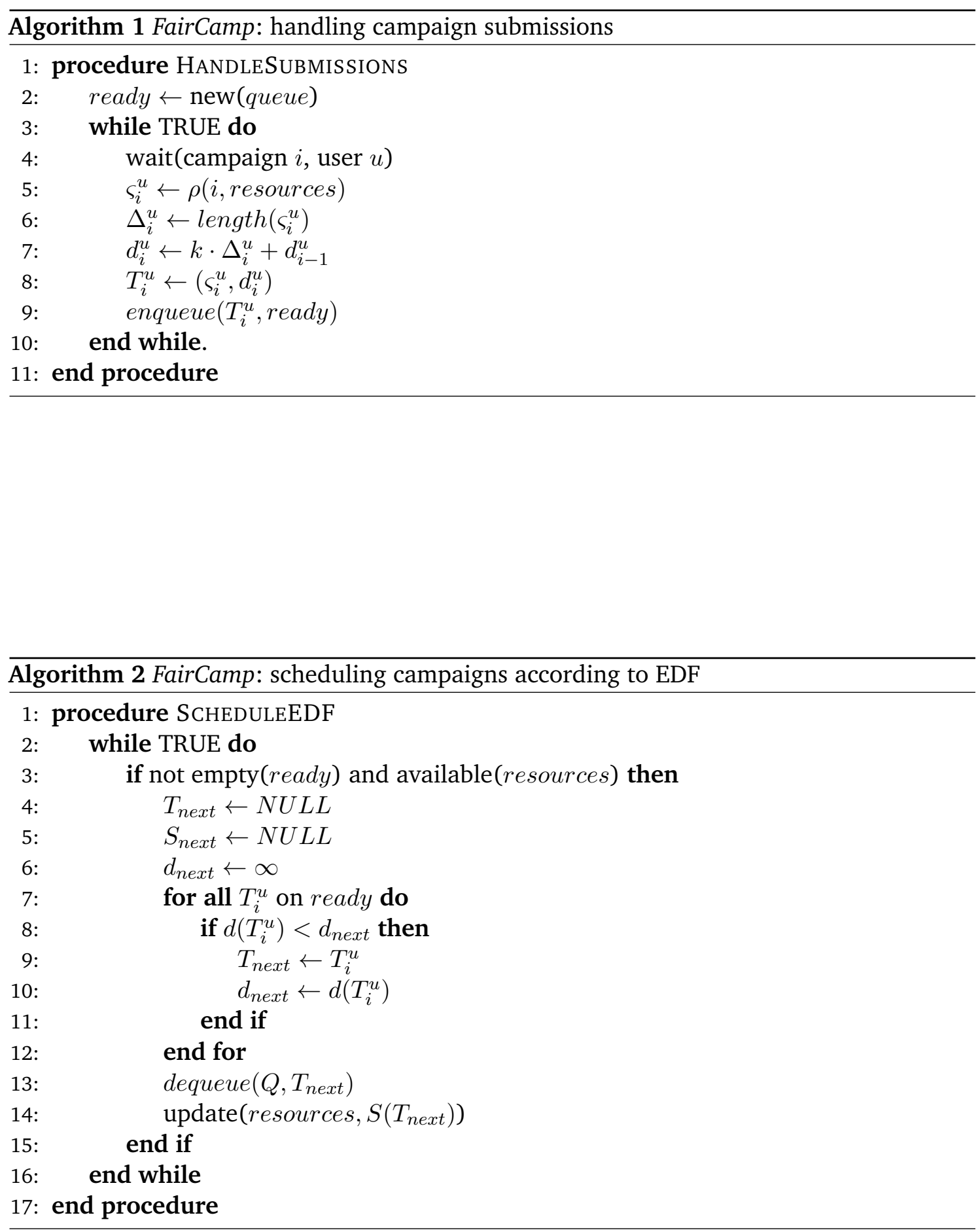
with the lowest deadline (lines 7-12). In the end, the chosen campaign schedule is placed on the resources (line 14).

\subsection{Feasibility of FairCamp}

FairCamp uses deadlines to guarantee fairness. Here, we show that a schedule produced by FairCamp is always feasible, i.e., all the deadlines are met. First, we assume that a campaign misses its deadline and then we show that this leads to a contradiction.

Proposition 4.1. In a schedule $S$ produced by FairCamp, all campaigns finish their executions before their respective deadlines.

Proof. Consider $m$ processors and $k$ users. For each campaign $i$, a schedule $\varsigma_{i}^{u}$ is generated whose length is $\Delta_{i}^{u}\left(\varsigma_{i}^{u}\right)$. This length is calculated by a $\rho$-approximation algorithm using all the processors. According to FairCamp, each campaign has deadline $d_{i}^{u}=k \cdot \Delta_{i}^{u}\left(\varsigma_{i}^{u}\right)+d_{i-1}^{u}=$ $k \cdot \Delta_{i}^{u}\left(\varsigma_{i}^{u}\right)+k \cdot \Delta_{i-1}^{u}\left(\varsigma_{i-1}^{u}\right) \ldots k \cdot \Delta_{1}^{u}\left(\varsigma_{1}^{u}\right)=k \cdot \sum_{i} \Delta_{i}^{u}\left(\varsigma_{i}^{u}\right)$. This deadline states that the length of each user workload can be stretched at a maximum factor of $k$. In other words, $d_{i}^{u}$ also can be seen as the deadline of the partial workload (from the first campaign until campaign i).

Now, consider a schedule $S$ constructed by FairCamp where at least one campaign misses its deadline and, without loss of generality, let campaign $i$ from user $u$ be the first campaign to miss its deadline on $S$. By definition, $d_{i}^{u}=k \cdot \sum_{i} \Delta_{i}^{u}\left(\varsigma_{i}^{u}\right)$, where $\sum_{i} \Delta_{i}^{u}\left(\varsigma_{i}^{u}\right)$ is the length of the (partial) workload issued from $u$.

Two conclusions can be observed from this scenario. First, the sum of the length of the workloads issued from the other $k-1$ users, between the beginning of the schedule $(t=0)$ and the beginning of campaign $i$, is bigger than $(k-1) \cdot \sum_{i} \Delta_{i}^{u}\left(\varsigma_{i}^{u}\right)$. Otherwise, the schedule would have an idle space, violating the campaign-compact constraint (Definition 3.1). All the campaigns after this idle space (including campaign $i$ ) could be shifted to end before $d_{i}^{u}$. So, more formally, 
- $\sum_{v \neq u}^{k} \sum_{j}^{\sigma^{v}} \Delta_{j}^{v}\left(\varsigma_{j}^{v}\right)>(k-1) \cdot \sum_{i} \Delta_{i}^{u}\left(\varsigma_{i}^{u}\right)$,

where $\sigma^{v}$ is the number of campaigns on each partial workload.

Second, the deadline of each partial workload is equal to or less than the deadline of $i$. More formally, $\forall v \neq u$ :

- $d_{\sigma^{v}}^{v} \leq d_{i}^{u}$

- $k \cdot \sum_{j}^{\sigma^{v}} \Delta_{j}^{v}\left(\varsigma_{j}^{v}\right) \leq k \cdot \sum_{i} \Delta_{i}^{u}\left(\varsigma_{i}^{u}\right)$

- $(k-1) \cdot \sum_{j}^{\sigma^{v}} \Delta_{j}^{v}\left(\varsigma_{j}^{v}\right) \leq(k-1) \cdot \sum_{i} \Delta_{i}^{u}\left(\varsigma_{i}^{u}\right)$.

But this clearly contradicts the first conclusion.

\subsection{Theoretical analysis}

We analyze FairCamp from two perspectives. First, Corollary 4.1 shows that FairCamp is efficient for an individual performance point of view, as $\sum \Delta_{i}^{u}$ is increased by at most $k \rho$ measured relatively to a system dedicated to the user. Second, Theorem 4.1 demonstrates that FairCamp is fair, as it is a $\rho$-approximation for minimizing the maximum stretch over all users.

Corollary 4.1. FairCamp is $(k \rho, \ldots, k \rho)$-approximation for $P|\operatorname{camp}| \sum \Delta_{i}^{u}$.

Proof. By definition, the length of a user workload execution is denoted by $\sum \Delta_{i}^{u}$. The deadline of a workload execution is the deadline of the last campaign $d_{\sigma^{u}}^{u}=k \cdot \sum_{i=1}^{\sigma^{u}} \Delta_{i}^{u}\left(\varsigma_{i}^{u}\right)=$ $k \rho \cdot \sum_{i=1}^{\sigma^{u}} \Delta_{i}^{u *}$, where $\sum_{i=1}^{\sigma^{u}} \Delta_{i}^{u *}$ is the optimal length of the workload. Since all deadlines are met, each workload is stretched by a factor of $k \rho$, at maximum.

Theorem 4.1. FairCamp is $\rho$-approximation for max-stretch that does not depend on $k$. 
Proof. Let us consider a solution $S$ constructed by FairCamp for an instance of $P|\operatorname{camp}| \Delta_{i}^{u}$. Now, consider without loss of generality, that the first $l$ scheduled campaigns belong to user $u$ while the campaign $l+1$ belongs to user $v$. Assume that $s_{l}$ is the start time and $C_{l}$ is completion time of campaign $l$. Similarly, $s_{l+1}$ and $C_{l+1}$ are the start and completion time of campaign $l+1$. As campaign $l+1$ starts immediately after campaign $l, s_{l+1}=C_{l}$. The arrival time of campaigns $l$ and $l+1$ are $t_{l}=s_{l}$ and $t_{l+1}=0$, respectively.

As $S$ was constructed by FairCamp, the deadline of $l+1$ is larger or equal to the deadline of $l$, otherwise, campaign $l+1$ would be scheduled earlier (after one of the $l$ first campaigns). So, $k \cdot \Delta_{l+1}^{v}\left(\varsigma_{l+1}^{v}\right) \geq k \cdot \sum_{1}^{l} \Delta_{i}^{u}\left(\varsigma_{i}^{u}\right)$. We also can denote this as $C_{l+1}-C_{l} \geq C_{l}$ or, more conveniently, $C_{l+1} \geq 2 C_{l}$.

Regarding only these $l+1$ campaigns, the stretch of user $u$ is $D^{u}=C_{l} / \sum_{1}^{l} \Delta_{i}^{u *}=$ $\sum_{1}^{l} \Delta_{i}^{u} / \sum_{1}^{l} \Delta_{i}^{u *}=\rho \sum_{1}^{l} \Delta_{i}^{u *} / \sum_{1}^{l} \Delta_{i}^{u *}=\rho$, while user $v$ has $\left.D^{v}=\left(C_{l+1}-t_{l+1}\right) / \Delta_{l+1}^{v *}\right)=$ $\rho\left(C_{l+1}-t_{l+1}\right) /\left(C_{l+1}-s_{l+1}\right)=\rho\left(C_{l+1}\right) /\left(C_{l+1}-C_{l}\right)$. As $\left(C_{l+1}\right) /\left(C_{l+1}-C_{l}\right)>1$, then $D^{v}>\rho$ and $\max \left(D^{u}, D^{v}\right)=D^{v}$.

Assume by contradiction that a better solution $S^{\prime}$ is achieved by changing the positions of campaigns $l$ and $l+1$. In this solution, the stretch of $u$ is $D^{\prime u}=\rho\left(C_{l+1}-s_{l}\right) /\left(C_{l}-s_{l}\right)$ and the stretch of user $v$ is $D^{\prime v}=\rho\left(C_{l+1}-C_{l}+s_{l}\right) /\left(C_{l+1}-C_{l}\right)$. For this solution to be better, both stretches of $S^{\prime}$ should be lower than $D^{v}$ :

- $D^{v}>D^{\prime v}$

- $\rho\left(C_{l+1}\right) /\left(C_{l+1}-C_{l}\right)>\rho\left(C_{l+1}-C_{l}+s_{l}\right) /\left(C_{l+1}-C_{l}\right)$

- $\left(C_{l+1}\right)>\left(C_{l+1}-C_{l}+s_{l}\right)$.

Since $C_{l}+s_{l}$ is positive, we proved that $D^{v}>D^{\prime v}$. Now, for $D^{\prime u}$ :

- $D^{v}>D^{\prime u}$

- $\rho\left(C_{l+1}\right) /\left(C_{l+1}-C_{l}\right)>\rho\left(C_{l+1}-s_{l}\right) /\left(C_{l}-s_{l}\right)$

- $C_{l+1}\left(C_{l}-s_{l}\right)>\left(C_{l+1}-s_{l}\right)\left(C_{l+1}-C_{l}\right)$

- $C_{l+1} C_{l}-C_{l+1} s_{l}>C_{l+1}^{2}-C_{l+1} C_{l}-C_{l+1} s_{l}+C_{l} s_{l}$ 
- $C_{l+1}<2 C_{l}-\left(C_{l} s_{l} / C_{l+1}\right)$.

But $C_{l+1} \geq 2 C_{l}$, so $D^{v}>D^{\prime u}$ is false which contradicts the assumption.

One can argue that considering only campaigns $l+1$ and $l$ causes the proof to lose its generality. But, in fact, we could place $l+1$ at any place before $l$ since this does not alter the new position of $l$ as well as the final contradiction found by the proof. Another point is that we are considering only the first $l+1$ campaigns from the beginning of the schedule, but the same logic applies if we consider any campaign completion time $C_{i}$ as the start point. Furthermore, changing the position of $l+1$ with a later campaign, would produce a stretch even bigger for campaign $l+1$ and, clearly, it does not produce a better solution.

\subsection{Simulations}

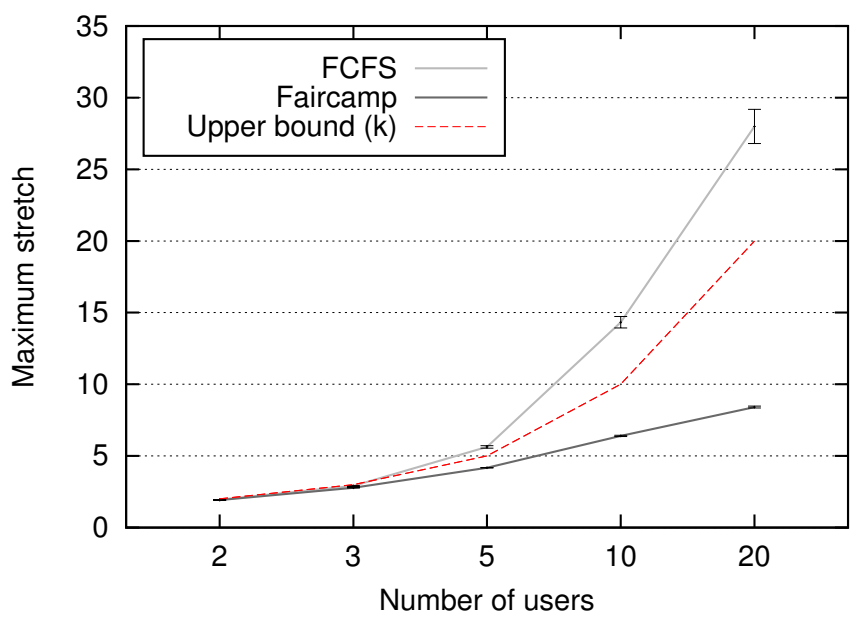

Figure 4.3: FCFS vs FairCamp; each point is an average over $10^{3}$ instances; error bars denote 95\% confidence intervals

In this section, we present a simulation demonstrating that, for the scenario considered in this chapter, FairCamp results in lower stretch values (and thus, better performance) than FCFS. The simulator plays the role of a centralized scheduler: it takes instances of user workloads as inputs; and it calculates the max-stretch obtained by each algorithm in an environment composed of $m=10$ identical processors.

Each instance is composed of $10^{4}$ jobs. For each job we set its length $p$ (uniformly taken 
from the range $[1,100])$. The job starts a new campaign with probability of 0.1 ; otherwise, it belongs to the previous campaign. If the job starts a new campaign, we set the owner of this campaign according to a Zipf distribution with exponent equal to 1.4267 , which best models submission behavior in large social distributed computing environment [IDE $\left.{ }^{+} 06\right]$.

We run $10^{3}$ instances for different number of users $(k): 2,3,5,10$ and 20 . The simulator runs both algorithms with the same instances. The results of the simulation are shown on Figure 4.3 and Figure 4.4. All results are presented with confidence level of 95\%.

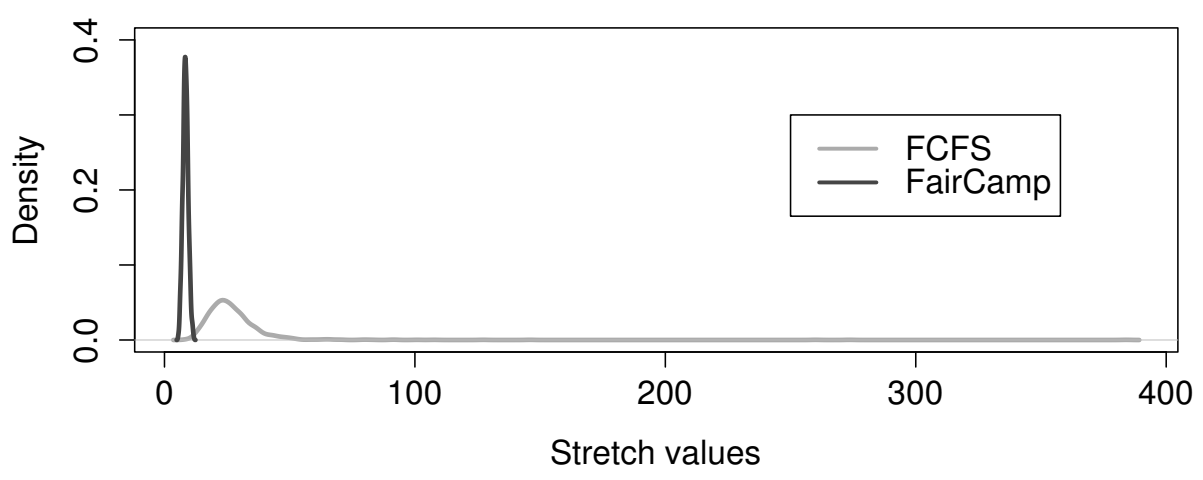

Figure 4.4: Max-stretch distribution for FCFS and FairCamp with 20 users

On Figure 4.3, the results for different number of users can be visualized. In this figure, a red dashed line is used to represent the theoretical upper bound $k$ of FairCamp algorithm. The first thing to note in this figure is that the max-stretch produced by FairCamp (solid line) is always below the upper bound.

Second, the results showed that, in systems with at least 5 users, FairCamp results in significantly lower max-stretch values than FCFS. With 20 users, the max-stretch of FairCamp is approximately 3.4 times lower. Good results are also achieved with 5 and 10 users, with improvements of 1.35 and 2.24, respectively. With few users, the difference is irrelevant.

This behavior is motivated by the Zipf distribution that assigns campaigns to their owners. According to Zipf's law, the most frequent user has a probability of being select that is twice the second most frequent user, three times the third most frequent user, and so on. The more users, the greater the difference in number of campaigns between the first 
user and the last one in the frequency rank. So, it is more likely that FCFS generates some schedules that will affect users with few campaigns and, consequently, will result in high values for the max-stretch.

Figure 4.4 is a density plot for the max-stretch values for FCFS and FairCamp with 20 users. For FairCamp all the max-stretch values falls between 5 and 13, the majority of them being around 8. For FCFS the majority of instances produces max-stretch values between 10 and 50. Nonetheless, for some instances, the max-stretches are extremely high, some of them reaching the hundreds. In fact, 7 of them fall between 100 and 400 . This happens because, despite its clear predominance around the average, FCFS is not bounded since it does not take stretch into account, like FairCamp.

In the next chapter, a solution is proposed in order to fit a more wide and realistic scenario. In this scenario users can join and leave the system ( $k$ is variable) and think-times values are positive and arbitrary. 


\section{Chapter 5}

\section{Fair online schedules: dynamic}

\section{scenario}

In real HPC systems, the number of users interacting with the resources is changing all the time. Users log in, execute processes, wait for results and may log out at any moment. Campaign submissions are unpredictable events as their executions are sparse, with idle time intervals between them. With those unpredictable events taking place, the challenge of delivering a fair scheduling is an even more complex endeavor, because the scheduler needs to maintain fairness only among the users that are present at each time moment.

In this chapter, a new algorithm called OStrich is proposed to deal with this new, more realistic, scenario. It follows the same principles of FairCamp, i.e., the fairness is achieved by assigning due dates to each campaign and user performance is bounded by his/her own workload and the number of users (although only "active" users are considered).

OStrich performance is assessed through simulating the replay of a workload trace from a real cluster, whereas this trace is composed of sequential jobs. In order to understand how to replay such traces, the simulations section is preceded by a discussion on how to detect campaigns in workloads, including the job dependencies inside campaigns. 


\subsection{The OStrich online algorithm}

The algorithm guarantees the worst-case stretch of each campaign $\left(D_{i}^{u}\right)$ to be proportional to the campaign's workload and the number of active users in the system. OStrich's principle is to create a virtual fair-sharing schedule that determines the execution priorities of the campaigns in the real schedule. The algorithm maintains a list of ready-to-execute campaigns ordered by their priorities and interactively selects the next job from the highest priority campaign. Any scheduling policy can be used to determine the execution order of jobs within a single campaign; for instance LPT [Gra69] (or, more appropriately, MLPT [Lee91]) or Shortest Processing Time (SPT) [BCS74].

The virtual fair-sharing schedule is maintained by dividing the processors between the active users at each given moment. The processors are divided evenly among the users, independently of users' submitted workload. The priority of a user's campaign is determined by its virtual completion time, i.e. the completion time in the virtual schedule. The campaign with the shortest virtual completion time has the highest priority for execution. This virtual completion time of a campaign $J_{i}^{u}$ is denoted by $\tilde{C}_{i}^{u}$ (more generally, we will use $\tilde{x}$ for denoting a variable $x$ in the virtual schedule). That way, if a user $u$ submits a campaign at time $t_{i}^{u}$, its virtual completion time is defined as the total workload of the campaign divided by its share of processors, added by its virtual start time. More formally:

$$
\tilde{C}_{i}^{u}(t)=\tilde{W}_{i}^{u} /(m / \tilde{k}(t))+\tilde{s}_{i}^{u}=\tilde{k}(t) \tilde{W}_{i}^{u} / m+\tilde{s}_{i}^{u}
$$

Note that the share of a user is defined as the number of processors $m$ divided by the number of active users at moment $t$, denoted by $\tilde{k}(t)$. Active users are those users that, according to the virtual schedule, have unfinished campaigns at time $t$. Formally, $\tilde{k}(t)$ is defined as $\tilde{k}(t)=\sum_{u}^{k} \mathbb{1}\{u, t\}$ where $\mathbb{1}\{u, t\}$ is an indicating function that returns 1 if $\exists i \mid \tilde{C}_{i}^{u}>t_{e}$ and 0 otherwise.

A campaign starts in the virtual schedule after its submission, but also not sooner than the virtual completion time of the previous campaign (the previous campaign can be completed earlier in the real schedule than in the virtual schedule). Formally, the virtual 
start time of a campaign is defined as

$$
\tilde{s}_{i}^{u}=\max \left(t_{i}^{u}, \tilde{C}_{i-1}^{u}\right)
$$

This condition guarantees that at each time moment, at most one campaign of each user is executing in the virtual schedule, as it happens on the real scheduler. Thus, the number of allocated processors depends on the number of active users, regardless the system load.

Additionally, OStrich does not allow a campaign to start its execution before its virtual start time $\left(s_{i}^{u} \geq \tilde{s}_{i}^{u}\right)$. This constraint keeps the real schedule in accordance with the fair principles of the virtual schedule: a user should not be able to take a greater share of the processors than what it was assigned in the virtual schedule. In other words, if a campaign starts at $\tilde{s}_{i}^{u}$ and finishes at $\tilde{C}_{i}^{u}$ on the virtual schedule, during this time interval its owner will not be able to execute jobs from another campaign. Furthermore, there is no incentive for any of the users to lie about or hide their private information from the other users. No advantage can be obtained by a user from splitting one campaign in many single-job-campaigns and submitting them separately.

The virtual completion time of the campaigns can be updated on two events: the submission of a new campaign and the completion of a campaign in the virtual schedule. These events may change the number of active users $(\tilde{k}(t))$ and, by definition, the virtual completion times of other active campaigns must also be modified.

Besides, at each event $e$ occurring at time $t_{e}$, the virtual workload of a campaign $\left(\tilde{W}_{i}^{u}\right)$ must be redefined based on how much it is left to be executed in the virtual schedule. The remaining workload of a campaign is defined by taking the time passed since the last event occurrence $t_{e-1}$ and multiplying it by the campaign's share of processors on that time interval. Considering all the events passed after the campaign's submission, the workload formula is $\tilde{W}_{i}^{u}=\sum_{j} p_{i, j}^{u}-\sum_{e}\left(m .\left(t_{e}-t_{e-1}\right) / \tilde{k}\left(t_{e-1}\right)\right)$. 


\subsection{Example}

Following, we have an example of how OStrich works. The Figure 5.1 shows the evolution of the real and the virtual schedule (labeled with ' $\mathrm{R}$ ' and ' $\mathrm{V}$ ' respectively) generated by the OStrich algorithm from $t=0$ to $t=18$ in a system with 6 identical processors (labeled from $M_{1}$ to $M_{6}$ ). This example shows 7 submissions issued from 3 different users: two submissions at time $t=0$ from users 1 and 2, two submissions at times $t=2$ and $t=5$ from user 3 , one submission at $t=8$ from user 2 and two submissions at $t=14$ from users 2 and 3.

In the real schedule, each box represents the execution of a job wherein the box label is the job length. In the virtual schedule, each box represents the load of a submitted campaign wherein the box label represents the load in terms of processing time. Each time the number of active users changes, the remaining workloads in the virtual schedule are redistributed. A plus signal is used to indicate when the remaining load should be added to the previous load in order to match the total campaign load. The list of ready campaigns is also represented on the example. This list contains all the submitted campaigns sorted accordingly to the owners priority. A callout is used to represent a campaign's submission. For a more didactic explanation of how OStrich works, the job executions are represented by 6 subfigures, labeled from $a$ to $f$. Also, each time a user campaign is finished, the max-stretch value is updated, if necessary.

At $t=0(a)$ the first campaigns of users 1 and 2 are submitted. From $t=0$ to $t=2$ ( $a$ and $b$ ), they are the only users in the system $(\tilde{k}(t)=2 ; 0 \leq t \leq 2$ ). The virtual schedule is constructed by sharing the processors equally between them and, according to definition (5.1), their virtual completion times are $\tilde{C}_{1}^{1}=16$ and $\tilde{C}_{1}^{2}=6$. The real schedule contains only jobs from user 2 since he/she has higher execution priority for having the smallest virtual completion time. 


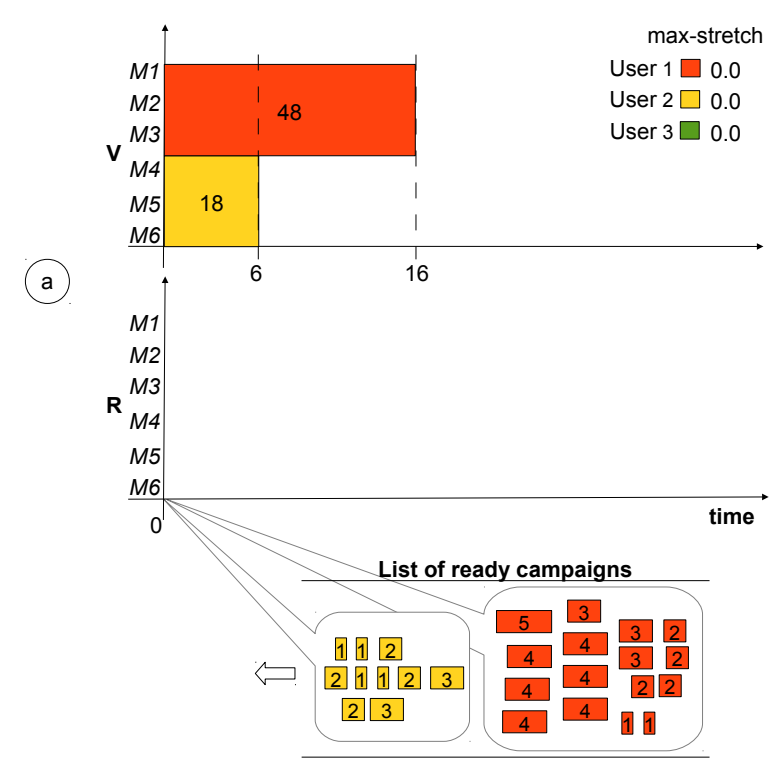

(c)

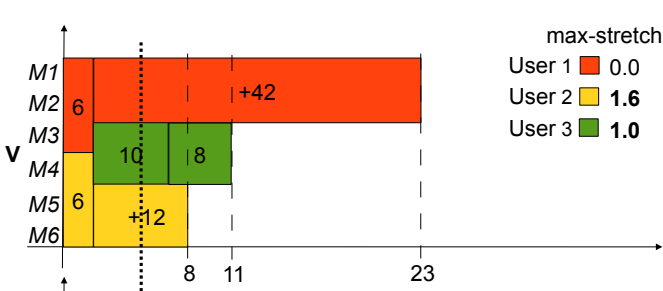

M1 2 1111

M2 222

\begin{tabular}{ll|l}
$M 3$ & 2 & 2 \\
\hline
\end{tabular}

M4 2 3

\begin{tabular}{ll|l:l} 
M5 & 3 & 1 & 5
\end{tabular}

\begin{tabular}{l|l|l:l} 
M6 & 3 & 1 & 4 \\
\hline
\end{tabular}

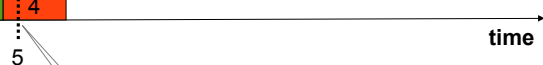

List of ready campaigns

(e)
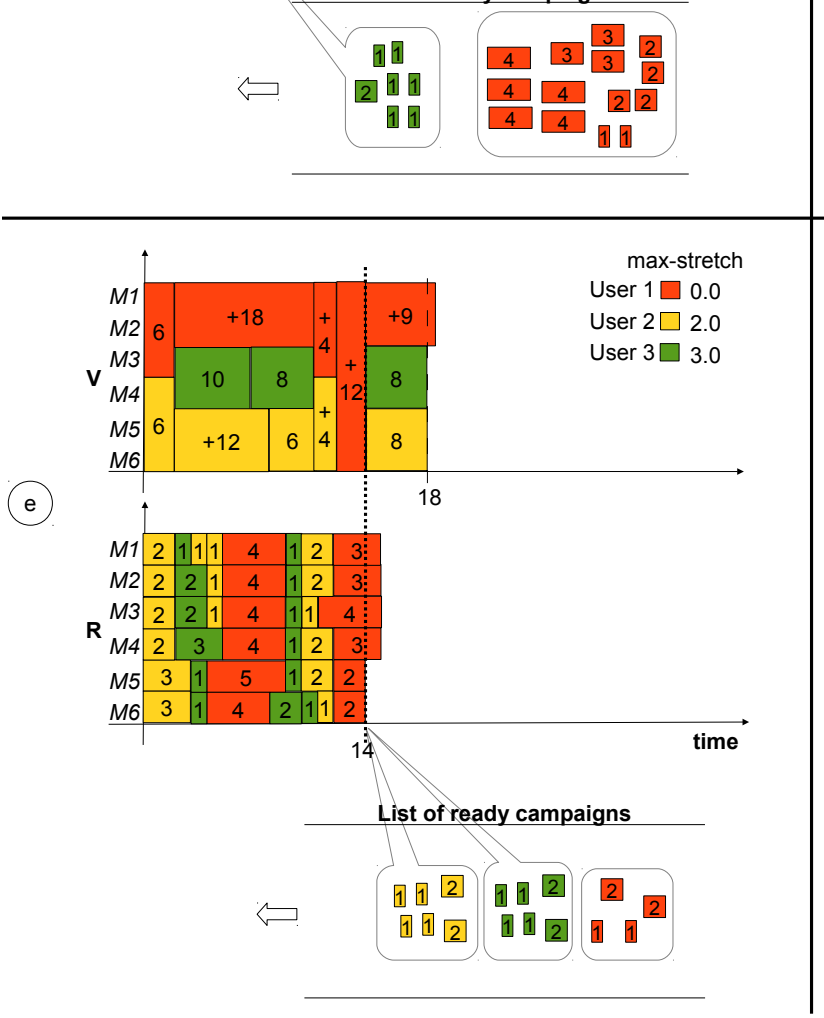

(b)

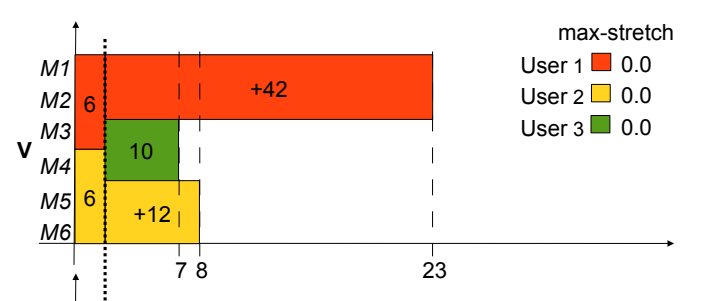

(d)
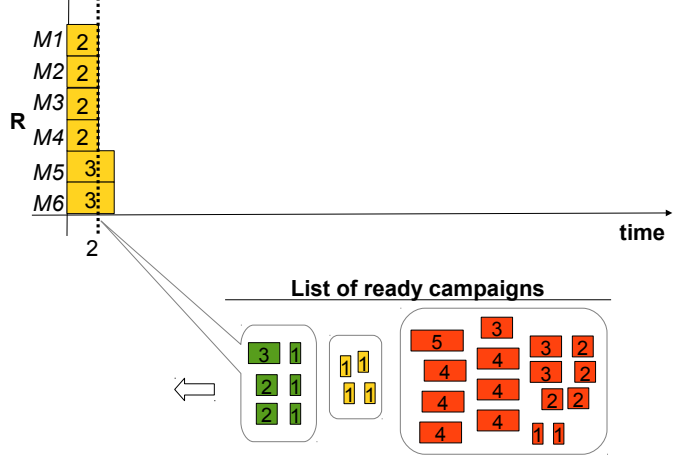

max-stretch User $1 \square 0.0$

User $2 \square 1.6$ User $3 \square 1.0$

(f)

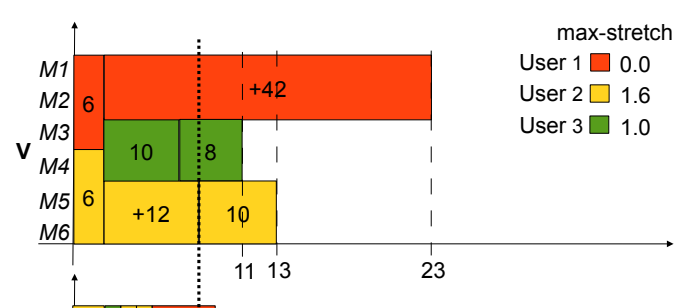

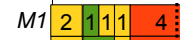

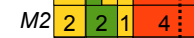

\begin{tabular}{l|l|l|l|l}
$M 3$ & 2 & 2 & 1 & 4 \\
\hline
\end{tabular}

\begin{tabular}{ll|c|c|c|}
\hline R & 2 & 2 & 3 & 4 \\
\hline
\end{tabular}

\begin{tabular}{l|l|l|l|}
\hline M5 & 3 & 1 & 5 \\
\hline
\end{tabular}

\begin{tabular}{l|l|l|l|} 
M6 & 3 & 1 & 4 \\
\hline & & &
\end{tabular}

time

List of ready campaigns

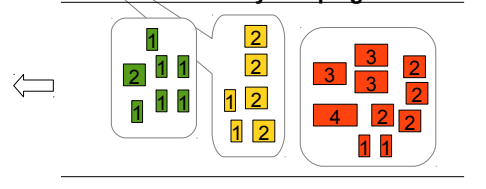

max-stretch

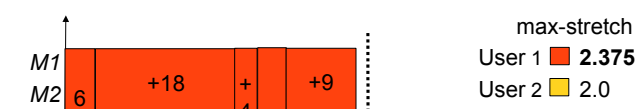

User $3 \square 3.0$

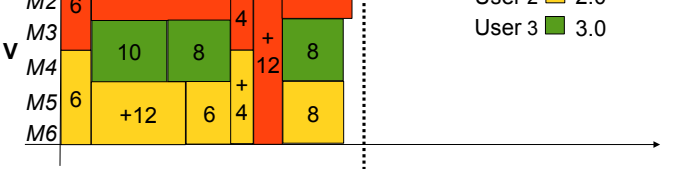

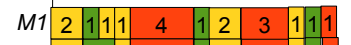

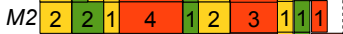

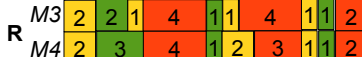

M5 \begin{tabular}{l|l|l|l|l|l|l|l|l} 
& 3 & 1 & 5 & 1 & 2 & 2 & 2 & 2 \\
\hline
\end{tabular}

\begin{tabular}{ll|l|l|l|l|l|l|l|l|l}
$M 6$ & 3 & 1 & 4 & 2 & 1 & 1 & 2 & 2 & 2 \\
\hline
\end{tabular}

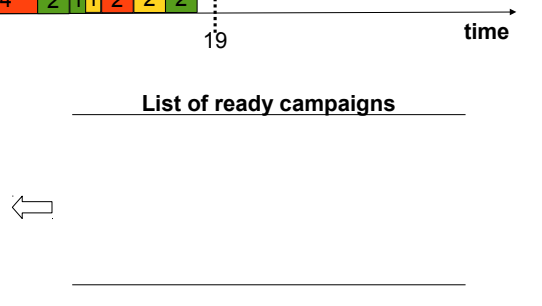

Figure 5.1: An example of the virtual and real schedule generated by the OStrich algorithm with 3 users 
The situation changes at $t=2$ when user 3 submits her/his first campaign (b). By this time, the users 1 and 2 had each a share of 6 in the virtual schedule but their virtual completion times were not exceeded. Now, the processors are equally shared between 3 users $(\tilde{k}(2)=3)$. The virtual completion time of user 3 is set to $\tilde{C}_{1}^{3}=7$ and the virtual completion times of users 1 and 2 are updated to $\tilde{C}_{1}^{1}=23$ and $\tilde{C}_{1}^{2}=8$, according to their remaining workloads in the virtual schedule.

Still at $t=2$, now, user 3 is the user with the highest priority. In the real schedule, the first campaign of user 2 is interrupted and some jobs from user 3 starts. However, note that the unfinished jobs from user 2 are not interrupted and so, in order to use processors $M_{5}$ and $M_{6}$, user 3 must wait until $t=3$.

From $t=2$ to $t=5$ ( $b$ and $c$ ) the first campaign of user 3 is finished and also the first campaign of user 2, as its remaining jobs are executed. Additionally, some jobs of the first campaigns of user 1 finally start to execute. At $t=5(\mathrm{c})$ the second campaign of user 3 is submitted, but note that the virtual completion time of her/his first campaign was not exceeded yet $\left(\tilde{C}_{1}^{3}=7\right)$. So, as $t_{2}^{3}<\tilde{C}_{1}^{3}$ and according to definition $5.2, \tilde{s}_{2}^{3}=\tilde{C}_{1}^{3}=8$. The virtual completion time of this new campaign is set to $11\left(\tilde{C}_{2}^{3}=11\right)$.

The user 3 now has the highest priority but, following the start time constraint of OStrich, her/his next campaign must wait until $\tilde{s}_{2}^{3}$ to became eligible to be executed. So, from $t=5$ to $t=8$ (c and d) only jobs from user 1 are executed. At $t=8$ (d) the second campaign of user 2 is submitted and its virtual completion time is set to 13 . User 3 remains having the highest priority and now his/her jobs start to be executed. From $t=8$ to $t=14$ ( $d$ and $e$ ) there are no new submissions, thus the already submitted campaigns of users 2 and 3 , and some jobs of user 1 , are executed accordingly to the priority order.

At $t=14(e)$, the third campaigns from users 2 and 3 are submitted. Now, users 2 and 3 have equal priorities (and higher than that of user 3). The campaign 1 of user 3 is interrupted and, from $t=14$ to 18 ( $e$ and $f$ ), these new campaigns are executed, followed, finally, for the remaining jobs of user 3 .

Visually, the result of OStrich is a schedule with campaigns being interleaved and executed in many pieces, according to the changing priorities between users. 


\subsection{Theoretical analysis}

In this section, the worst case stretch of a campaign is analyzed. The idea for the proof is to bound the completion time of the last job of a campaign using a "global area" argument compared to the virtual schedule. In this proof, $p_{\max }$ denotes the maximum job length in the system. "Area" is a measure in terms of amount of time $\times$ number of processors.

The virtual schedule is denoted by $\mathrm{V}$ and the real schedule by R. To simplify the formulation of the proofs of this section, it is said that the virtual schedule V "executes an area", even though $\mathrm{V}$ is just an abstraction used for prioritizing real jobs. At time $t$, an area is being "executed" by V if in V there is a fraction of processors assigned to this area.

\subsubsection{Worst-case bound}

As $\mathrm{V}$ can assign a job an arbitrary fraction of processors (from $\epsilon$ to $m$ ), a schedule in $\mathrm{V}$ is represented by horizontal load streams, one for each active user. Idle intervals can be present in $\mathrm{V}$ only when there are no ready jobs to be executed. In turn, $\mathrm{R}$ must execute each job on a single processor, thus some processors can be idle even when there are ready jobs. This can happen when $t_{i}^{u}<\tilde{s}_{i}^{u}$ and the ready jobs of campaign $J_{i}^{u}$ must wait until $t=\tilde{s}_{i}^{u}$. So, the question is whether the idle times that might additionally appear in R can cause a systematic delay of $\mathrm{R}$ compared to $\mathrm{V}$. The following lemma shows that once $\mathrm{R}$ is delayed by an area of $m p_{\max }$, the delay does not grow further, as there is always a ready job to be executed.

The lemma considers only the idle time in the "middle" of the scheduling chart, i.e., after the start time of the first job and up to the start time of the last job; this is sufficient to characterize the online behavior of OStrich.

Lemma 5.1. The total idle area in $R$ (counted from the earliest to the latest job start time) exceeds the total idle area in $V$ by at most $m p_{\text {max }}$.

Proof. Consider first a V schedule with no idle times. Assume by contradiction that $t$ is the first time moment when the total idle area in $\mathrm{R}$ starts to exceed $m p_{\max }$. Thus, at least one processor is free at time $t$ and there is no ready jobs to be executed. As V has no idle times, 
at time $t$ the area executed by $\mathrm{V}$ exceeds the area executed by $\mathrm{R}$ by more than $m p_{\max }$. Thus, the area exceeding $m p_{\max }$ is ready to be executed at $\mathrm{R}$ : as a single job has an area of at most $p_{\max }$, an area of $m p_{\max }$ is composed of at least $m$ jobs. Thus, at least $m$ jobs are being executed, or ready to be executed in $\mathrm{R}$ at $t$. This contradicts the assumption that there is at least one free processor in $\mathrm{R}$ at time $t$.

If there is idle time in $\mathrm{V}$, it can be replaced by a set of dummy jobs $J_{I}$ that are "executed" by V, but not necessarily (or not completely executed) by R. If R executes $J_{I}$ entirely, the lemma is true, and this can be proved using the same argument of the previous paragraph, since $J_{I}$ "contributes" with the same amount of idle area $\left(\sum p_{I}\right)$ for $\mathrm{V}$ as well as for R. If $\mathrm{R}$ executes $J_{I}$ partially (i.e. it executes a set $J_{I}^{\prime}$, where $0 \leq p_{I}^{\prime} \leq p_{I}$ ), the contribution of these jobs is smaller to the idle area of $\mathrm{R}$ than to the idle area of $\mathrm{V}\left(\sum p_{I}^{\prime} \leq \sum p_{I}\right)$.

R starts to execute jobs from campaign $J_{i}^{u}$ when this campaign has the shortest completion time in V. Yet, it is possible that after some jobs from $J_{i}^{u}$ have started, another user $v$ submits her/his campaign $J_{j}^{v}$. If $J_{j}^{v}$ has a smaller area than what remains of $J_{i}^{u}$, it gains a higher priority. In this case, $J_{i}^{u}$ would be executed in $\mathrm{R}$ in so-called pieces: two jobs $J_{k}, J_{l} \in J_{i}^{u}$ belong to the same piece iff no job from other campaign $J_{j}^{v}$ starts between them. That is, $\nexists J^{\prime}:\left(J \in J_{j}^{v}\right) \wedge\left(s_{J k}<s_{J^{\prime}}<s_{J l}\right)$.

The following lemma bounds the completion time of the last piece of the campaign. After a campaign is completed in the virtual schedule, it cannot be delayed by any other newly-submitted campaign; thus it has the highest priority and its remaining jobs are executed in one piece (i.e., the last piece). The lemma upper-bounds the virtual area with the higher priority by the area of the campaign, given that, in the worst case, $k$ users submit campaigns of equal area at the same time. In this case, they would end at the same time in $\mathrm{V}$ and be executed in arbitrary order in $\mathrm{R}$.

Lemma 5.2. The completion time $C_{i, q}^{u}$ of the last piece $q$ of a campaign $J_{i}^{u}$ is bounded by a sum:

$$
C_{i, q}^{u} \leq t_{i}^{u}+k \frac{W_{i-1}^{u}}{m}+p_{\max }+(k-1) \frac{W_{i}^{u}}{m}+p_{\max }+\frac{W_{i}^{u}}{m}+p_{\max }^{u}
$$

Proof. In the expression (5.3), $t_{i}^{u}+k W_{i-1}^{u} / m$ expresses the maximum time a campaign 


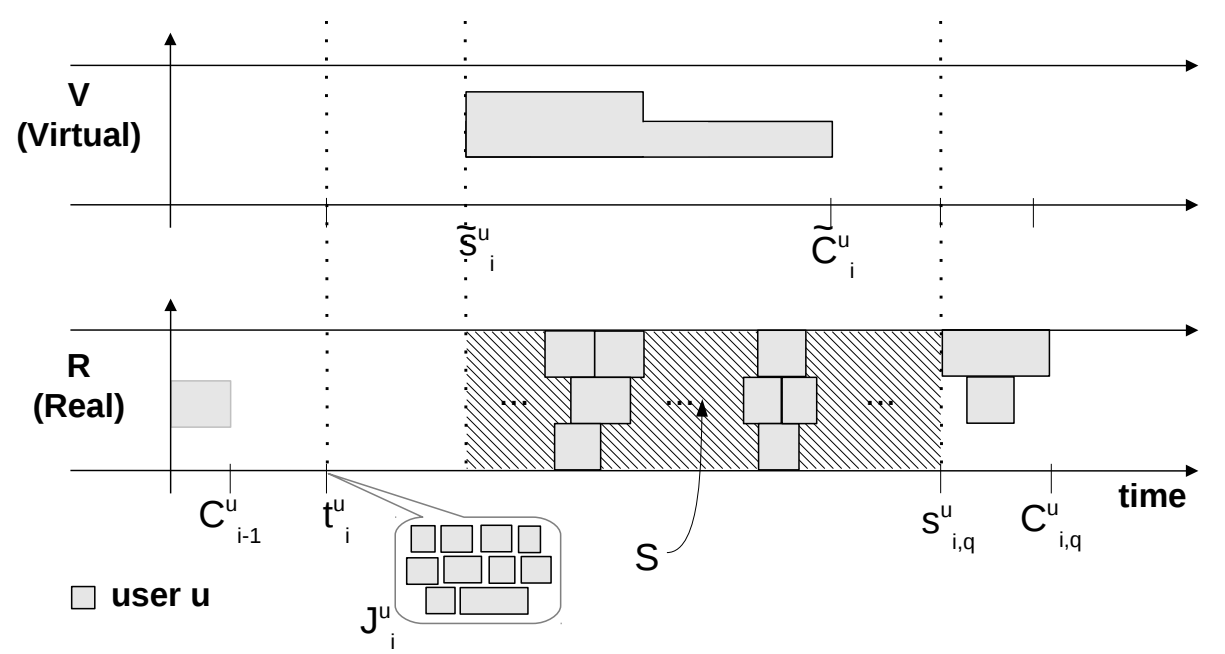

Figure 5.2: Analysis of OStrich: bound for the campaign stretch

may wait until the virtual completion time of the previous campaign $J_{i-1}^{u}$ of the same user; $(k-1) W_{i}^{u} / m$ bounds the time needed to execute other users' campaigns that can have higher priority; $W_{i}^{u} / m+p_{\max }^{u}$ bounds the execution time of the campaign $J_{i}^{u}$; and two $p_{\max }$ elements represent the maximum lateness of $\mathrm{R}$ compared to $\mathrm{V}$ and the maximum time needed to claim all the processors.

From definitions (5.1) and (5.2), and knowing that $t_{i}^{u} \geq \tilde{s}_{i-1}^{u}$ (i.e. the next campaign cannot be released before the previous one starts), we obtain $\tilde{s}_{i}^{u} \leq t_{i}^{u}+k W_{i-1}^{u} / m$.

There is no idle time in $\mathrm{R}$ in the period $\left[\tilde{s}_{i}^{u}, s_{i, q}^{u}\right)$, otherwise, the last piece could have been started earlier. We denote by $A$ the area of jobs executed in $\mathrm{R}$ after the time moment $\tilde{s}_{i}^{u}$ and until $\tilde{s}_{i, q}^{u}$. We claim that $A \leq m p_{\max }+(k-1) W_{i}^{u}+W_{i}^{\prime u}$, where $W_{i}^{\prime u}$ is the area of jobs from campaign $J_{i}^{u}$ executed until $s_{i, q}^{u}$. The Figure 5.2 facilitates the visualization of these notations, including the area $A$ (shaded area).

To prove the claim, we analyze job $J$ executed in R in the period $\left[\tilde{s}_{i}^{u}, s_{i, q}^{u}\right)$. First, $J$ is not executed in $\mathrm{V}$ after $s_{i, q}^{u}$. If $J$ were in $\mathrm{V}$ after $s_{i, q}^{u}, J$ would have lower priority than jobs from campaign $J_{i}^{u}$, so OStrich would not choose $J$ over jobs from campaign $J_{i}^{u}$.

Second, if $J$ is executed in V before $\tilde{s}_{i}^{u}$, it means that R is "late" in terms of executed area: but the total area of such "late" jobs it at most $m p_{\max }$ (from Lemma 5.1).

Thus, if $J$ has a corresponding area in the virtual schedule executed in the period $\left[\tilde{s}_{i}^{u}, s_{i, q}^{u}\right)$, the area $A$ of the jobs started in the real schedule in this period is equal to the 
area of the virtual schedule between $\left[\tilde{s}_{i}^{u}, s_{i, q}^{u}\right)$ plus the lateness $m p_{\max }$. Recall that from time $s_{i, q}^{u}$ till the start of the last job of $J_{i}^{u}$, the campaign $J_{i}^{u}$ has the highest priority (as it is not interrupted by any other campaign). Thus, at the latest, $s_{i, q}^{u}$ corresponds to the time moment $\tilde{C}_{i}^{u}$ in the virtual schedule when the campaign $J_{i}^{u}$ completes (plus the lateness $\left.p_{\max }\right)$. By definition of the virtual schedule, $s_{i, q}^{u} \leq p_{\max }+\tilde{s}_{i}^{u}+k \frac{W_{i}^{u}}{m}$.

Starting from $s_{i, q}^{u}$, the remaining jobs of $J_{i}^{u}$ start and complete. $J_{i}^{u}$ can claim all processors at the latest $p_{\max }$ after $s_{i, q}^{u}$. Then, by using classic lower bounds, it takes $W_{i}^{u} / m+p_{\max }^{u}$ to complete the campaign.

The following theorem states that in OStrich the stretch of a campaign depends only on the number of active users and the relative area of two consecutive campaigns.

Theorem 5.1. The stretch of a campaign is proportional to the number of active users $k^{1}$ and the relative area of consecutive campaigns. $D_{i}^{u} \in O\left(k\left(1+\frac{W_{i-1}^{u}}{W_{i}^{u}}\right)\right)$.

Proof. The result follows directly from Lemma 5.2. Recall that, for campaign $J_{i}^{u}$, the stretch $D_{i}^{u}$ is defined by $D_{i}^{u}=\left(C_{i}^{u}-t_{i}^{u}\right) / l_{i}^{u}=\left(C_{i}^{u}-t_{i}^{u}\right) / \max \left(W_{i}^{u} / m, p_{\max }^{u}\right)$. Also, $C_{i}^{u}=C_{i, q}^{u}$, i.e. the completion time of a campaign is equal to the completion time of its last "piece". Replacing $C_{i}^{u}$ by the definition of $C_{i, q}^{u}$ taken from Lemma 5.2, we have

$$
D_{i}^{u} \leq \frac{\frac{k W_{i-1}^{u}}{m}+3 p_{\max }+\frac{k W_{i}^{u}}{m}}{\max \left(W_{i}^{u} / m, p_{\max }^{u}\right)} \leq \frac{\frac{k W_{i-1}^{u}}{m}+3 p_{\max }+\frac{k W_{i}^{u}}{m}}{W_{i}^{u} / m} \leq k\left(1+\frac{W_{i-1}^{u}}{W_{i}^{u}}\right)+3 m p_{\max }
$$

For a given supercomputer, $m$ is constant; similarly, the maximum size of a job $p_{\max }$ can be treated as constant, as it is typically limited by system administrators. Hence, $D_{i}^{u} \in O\left(k\left(1+W_{i-1}^{u} / W_{i}^{u}\right)\right)$.

It is worth noting that the stretch does not depends on the current total load of the system. Heavily-loaded users do not influence the stretch of less-loaded ones. Also, this bound is tight as we will see next.

\footnotetext{
${ }^{1}$ The number of active users may vary on time. Here, we assume that $k$ is the biggest value it assumes during the execution of the campaign.
} 


\subsubsection{Tightness}

This section analyzes the tightness of the $O\left(k\left(1+\frac{W_{i-1}^{u}}{W_{i}^{u}}\right)\right)$ bound proposed in Theorem 5.1. We start with a negative result that says that in heavily-loaded systems, campaigns have to be executed sequentially, thus at least one of them will have a stretch in $O(k)$.

Proposition 5.1. No scheduling algorithm can achieve better stretch than $O(k)$.

Proof. Consider an instance with $k$ users, each submitting at $t=0$ a campaign with $m$ jobs of unit size. The campaigns have to be executed sequentially, so there is at least one user whose campaign completes at time $k$.

The following proposition shows an instance in which the bound $O\left(k\left(1+\frac{W_{i-1}^{u}}{W_{i}^{u}}\right)\right)$ (Theorem 5.1) is asymptotically tight. The instance is composed of a series of long campaigns followed by a series of short campaigns. A user who had her/his long campaign executed at the beginning, must wait with the short campaign not only the time needed to complete all other long campaigns, but also possibly other short campaigns.

Proposition 5.2. The bound $D_{i}^{u} \in O\left(k\left(1+\frac{W_{i-1}^{u}}{W_{i}^{u}}\right)\right)$ is asymptotically tight.

Proof. Consider an instance with $m$ processors and $k$ users having two campaigns each. At $t=0$, each user submits a campaign with $m$ jobs of size $p_{\max }$. The second campaign of a user is submitted immediately after the completion of the first campaign, with $m$ jobs of size $p=1$. As campaigns $\left\{J_{1}^{u}\right\}$ have the same priority; and campaigns $\left\{J_{2}^{u}\right\}$ have the same priority, OStrich can produce any schedule that executes first all the campaigns $\left\{J_{1}^{u}\right\}$, then all campaigns $\left\{J_{2}^{u}\right\}$. Thus, an admissible schedule is $\left(J_{1}^{1}, J_{1}^{2}, \ldots, J_{1}^{(k)}, J_{2}^{(k)}, J_{2}^{(k-1)}, \ldots, J_{2}^{1}\right)$. The completion time of $J_{2}^{1}$ is $C_{2}^{1}=k p_{\max }+k$, thus the stretch $D_{2}^{1}=(k-1) p_{\max }+k=$ $1+(k-1)\left(1+\frac{W_{1}^{1}}{W_{2}^{1}}\right)$.

\subsection{Analysis of campaigns in workloads}

In this section, we describe how to detect campaigns composed of sequential jobs in workloads from real traces of production clusters. 


\subsubsection{Workload modeling}

In recent works about workload modeling [Fei05, ZF12, MF12], Feitelson et al. consider that the action of a user (i.e. his/her job submission behavior and frequency) is influenced by the actions of the other users and by the scheduler decisions. This user feedback has an impact on the observed workload.

This is justified by the fact that the user is aware of the current system behavior and his future actions directly depend on the system's actual performance. To incorporate this feedback in the model, Feitelson proposes the postulate of dependencies between jobs, which relies upon the idea of what he calls the think-time, i.e. the delay between the ending of a job and the submission of a new one. This delay represents the time taken by the user to analyze the result of a executed job and to prepare the submission of the next. This is the same concept as the think-time in the Campaign Scheduling model.

In [ZF12], the think-time is used to detect user sessions and batches in a workload trace. A user session is a period of continuous work where the user submits jobs. The continuity of a session is defined by a time threshold. Interruption of user activities above this threshold configures a session break. Within a session, jobs that are overlapping will be grouped in what is called a batch. Note that, the notion of campaign is similar to the notion of batch: a set of jobs, submitted asynchronously by a user and that will run independently from the others.

Still on [ZF12], different methods are proposed to detect users' batches in a workload log. The LAST algorithm was originally proposed in [SF06]. In this approach, two jobs that are submitted one after the other belong to the same batch if the last one was submitted before the end of the first one. The ARRIVAL algorithm is based on the inter-arrival times of the jobs, i.e. it does not take into account their runtimes. The MAX algorithm states that a job belongs to a batch if it overlaps at least one of the jobs of the batch. According to comparisons, the results showed that the choice of which algorithm should be adopted is not general. The ARRIVAL algorithm, for example, is more adequate for web search engine logs, as it was concluded at [MF12].

In this thesis, the MAX algorithm is adopted since it focus on task runtime and thus is 
more suitable for HPC systems. Furthermore, the MAX algorithm produces batches that correspond to the concept of campaign described in the model.

\subsubsection{Example}

Figure 5.3 shows an example of a campaign detected using the MAX algorithm. The algorithm is simple: for each job, it looks whether it belongs to the current campaign or initiates a new one. A job belongs to the current campaign if its submission time is prior to the completion time of the last job to end in the campaign (i.e. the maximum completion time so far in the user workload).

So, following the example in the figure, the job (1) starts a new campaign either because it is the first submitted job or its submission time comes after the maximum completion time previously defined. The maximum completion time is updated to the finish time of (1). Next, the submission time of (2) is compared against this value and, being previous, (2) is included in the same campaign of (1). Now, the maximum completion time is updated to the finish time of (2). And so on, until the inclusion of the last task (7).

Note that, after the inclusion of (4) and (5), the maximum completion time remains as the finish time of (3) since the finish times of (4) and (5) are lower. Note also that, without task (6), there would be two campaigns: the first one comprising jobs from (1) to (5) and the second one comprising only job (7). Thus, task (6) works as a "linker job".

It may happen a situation with several small and disconnected jobs acting as a single campaign due to the presence of a big linker job overlapping with them. In practice, this can be a problem when it is clear that two or more jobs should be assigned to distinct campaigns. However, it is very difficult to detect and split this kind of campaigns. In the example of the figure, it is not deterministic to define the correct campaign set. It is not clear, for example, whether the job (6) belongs to the campaign of jobs (1) to (5) or to the campaign of job (7). Thus we have no choice other than set all these jobs in the same campaign. However, it would be unrealistic to consider all the jobs of a campaign as independent. The job (7) is submitted after all the jobs in the campaign except (6). Although not explicitly declared whether (7) depends or not on the other jobs, the execution 
order of jobs should be respected in order to define a worst-case assumption. We can solve this by setting inter-job dependencies.

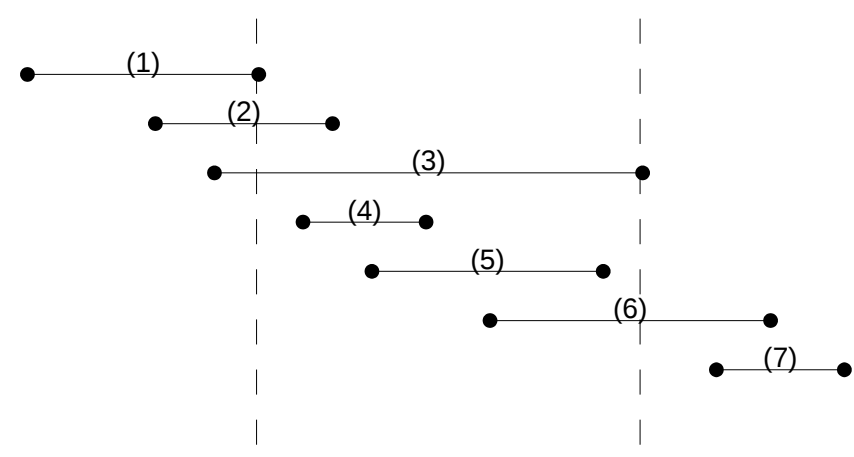

Figure 5.3: An example of a campaign with seven jobs according to the MAX algorithm

\subsubsection{Interval graphs for job dependencies}

The MAX algorithm is used to detect campaigns but not the internal job dependencies inside campaigns. This can be done using interval graphs [Pap78] to describe the precedence relations between jobs. Thus, for each campaign, we create an interval graph of all the jobs that make up the campaign. In this graph, the jobs are the vertices and two vertices are linked by an edge if the corresponding jobs overlap (between their submission time and end time). Then, from this interval graph we build the dependency graph presented in Figure 5.4. A job will have as dependencies all the jobs terminated before its submission. Finally, we apply the transitive reduction on the dependency graph to remove transitive edges.

(3)

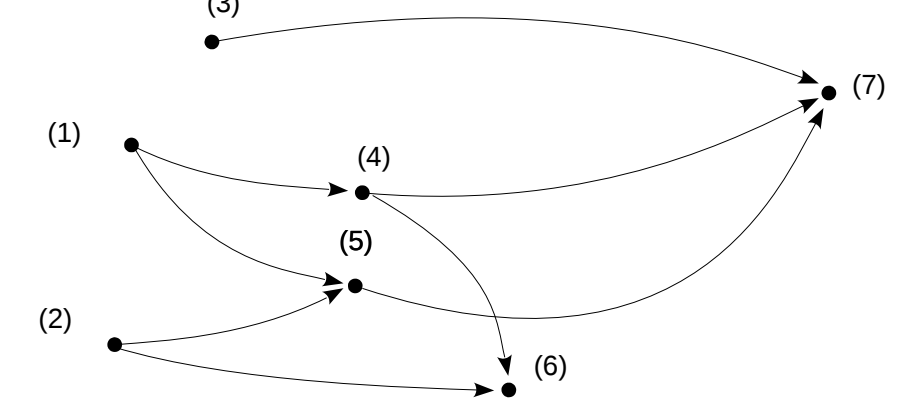

Figure 5.4: A Directed Acyclic Graph (DAG) illustrating dependencies inside the campaign (same campaign of Figure 5.3) 


\subsubsection{Campaigns with dependencies: a formal model}

The former campaign model, introduced in Chapter 3, allowed to release any job of a campaign when the campaign starts. Because of the way we detect campaigns (MAX algorithm from [ZF12]), this induces a bias.

The MAX method, cannot guarantee that all the jobs in the campaign are overlapping. In this case some jobs that stay in the system longer than the others behave as "linkers" and group together in the same campaign jobs that do not run at the same time. Thus, in the same campaign some jobs can start after another job's termination. To guarantee that we respect that constraint in the replay, we set up job dependencies during the campaign detection. A job will have as dependencies all the jobs in the campaign terminated before its submission.

OStrich can be extended to handle job dependencies: the jobs of a campaign are primarily sorted by their number of immediate successors on decreasing order and secondly by their length as in LPT [Gra69]. When a job is executed, it may trigger its successors to become ready for execution. But if the highest priority campaign has no ready jobs, the second highest priority campaign is chosen and so on.

\subsection{Simulations}

In this section, we analyze the performance of OStrich on a workload trace taken from a real cluster machine. First, we describe the settings of the simulations and then we compare the performance of OStrich to the standard scheduler (Maui with FCFS and backfilling) used on this cluster.

To run the simulations, we choose the LPC-EGEE ${ }^{2}$ trace from the Parallel Workload Archive [Fei] (cleaned version). This trace comes from a cluster that is part of the EGEE ${ }^{3}$ project and has the particularity of being composed of multiple Bag-of-Tasks (i.e. serial jobs), which fits well the concept of a campaign. The scheduler used to produce the execution trace was Maui [JSC01] with its default EASY configuration, that is, FCFS with

\footnotetext{
${ }^{2}$ http://www.cs.huji.ac.il/labs/parallel/workload/1_lpc/index.html

${ }^{3}$ http://public.eu-egee.org/intro/
} 
backfilling. However, since all the jobs are sequential, backfilling does not improves the makespan: whenever a CPU is available, this is enough for executing the job at the head of the wait-queue, and thus no other job is allowed to skip it.

The trace is long (10 months); it contains several cuts (due to electrical problems, management system reconfiguration, cooling failures, etc) and machine failures. The system is composed of 140 identical machines. The performance of OStrich is compared to the one extracted from the log.

During the replay of a real workload trace, we need to be cautious in removing the downtimes. During such periods, no new jobs were submitted and no jobs were running. Thus, the schedule of the original trace was strongly impacted by this. If we replay the whole workload without taking into account these downtimes, it creates "desynchronization" points where the new schedule, which is not aware of the downtimes, will take benefit from this surplus of time.

To solve this, we propose to split the original workload into several pieces between two downtime periods. Each sub-part of the workload will of course keep the information of the jobs that were queued before the failure. This guarantees that no jobs are omitted during the replay. Each piece of the workload will then be replayed separately in the simulator. The global analysis is done from the aggregation of all the replay results of these sub-parts. In total, the workload contains 56918 campaigns issued from 55 users.

First, we analyze the distribution of stretch values obtained by each campaign. The results are reported in Figures 5.5 and 5.6. For these figures, a step of 0.15 has been chosen to draw the histogram as it is precise enough to see correctly the shape of the distribution without adding too much noise.

While in the original log the stretch values are dispersed in the range $[1,3]$, with OStrich the vast majority of values ( $80 \%$ of the campaigns) are near the optimal value of 1 (between 1 and 1.15). More precisely, 68.6\% values were optimal (a stretch of 1) and 89.7\% were below 1.5 with OStrich. In the original log, the percentage of optimal values was only $0.3 \%$ with $55.2 \%$ of the values under 1.5 . Also important, we can see that high stretches values are very few in OStrich. 


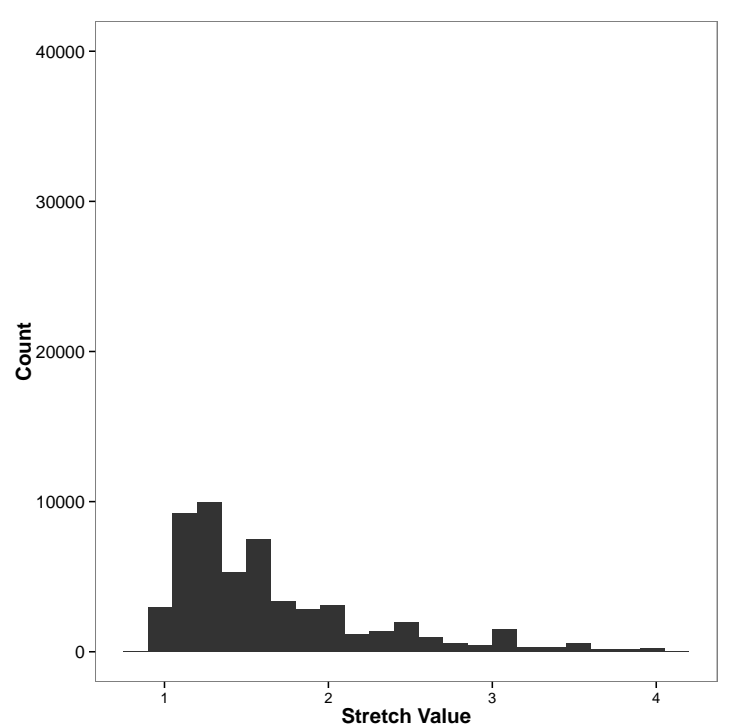

Figure 5.5: Campaign stretch values distribution (original log: Maui, FCFS)

Figures 5.7 and 5.8 show the system utilization, or the percentage of allocated resources over time. The results of both algorithms look similar but it is worth to note that, with OStrich, the system achieves its maximum utilization more often and more periods where the system is empty are visible. This is interesting because OStrich maximizes the utilization when there are jobs to process and thus creates periods of non-utilization of the platform that can be used for Energy-Saving strategies, for example.

In order to obtain a more detailed look of the system utilization, we zoom into these results (figures 5.9 and 5.10) to analyze an interval of two months (December and January). These months were chosen due to their high rate of system utilization. The same observation remains valid: with OStrich, peak usage activity is more frequent, and, unlike the original $\log$, it achieves $100 \%$ of resource utilization many times.

The precedence relations between jobs is obviously an obstacle to optimization: without this, the level of parallelism would be higher and better stretch values could be obtained. Thus, in theory, the absence of dependencies between jobs would increase system utilization. The results in Figure 5.11 confirm this claim. This figure shows the system utilization of the same period (December and January) in an OStrich schedule with no job dependencies. In this scenario, the system utilization is more homogeneous. Regarding the periods where the system load is above $10 \%$, this scenario achieves system utilization at least $20 \%$ greater 


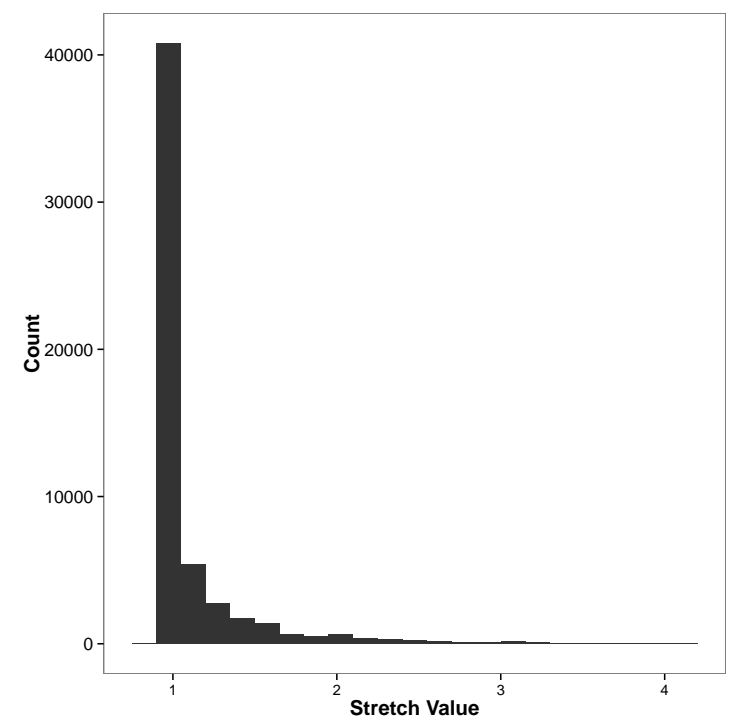

Figure 5.6: Campaign stretch values distribution (OStrich)

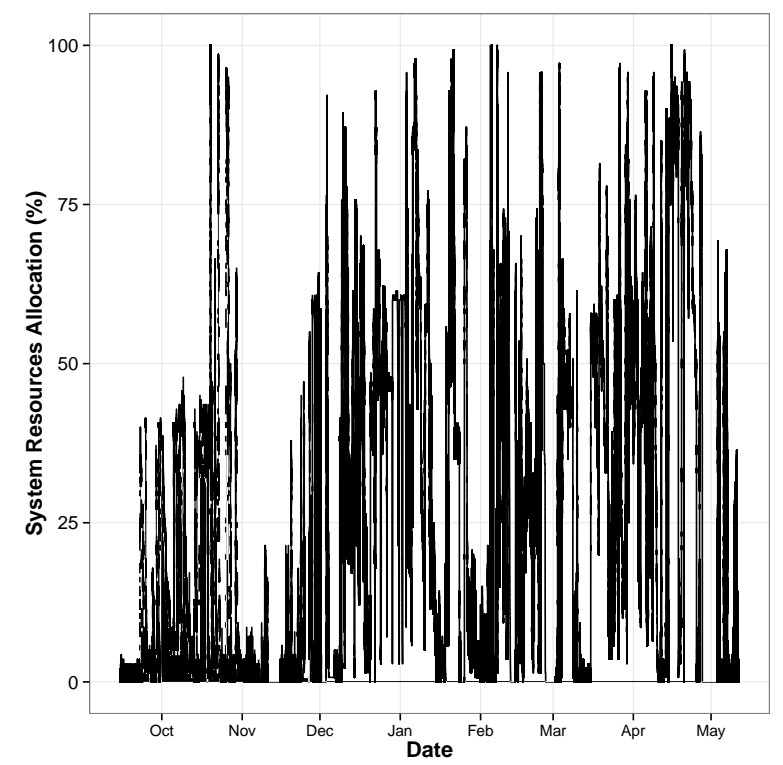

Figure 5.7: System resources utilization for whole trace (original log: Maui, FCFS)

than the one obtained with job dependencies. Not surprisingly, periods of user inactivity are also more frequent.

The comparison of the two approaches of campaign detection, with and without adding dependencies, is also interesting. Regarding the original workload shape, we can observe that the replay with the no-dependencies approach is more biased. Whereas Figure 5.10 is quite similar to Figure 5.9, Figure 5.11 shows a scenario where the utilization is better due 


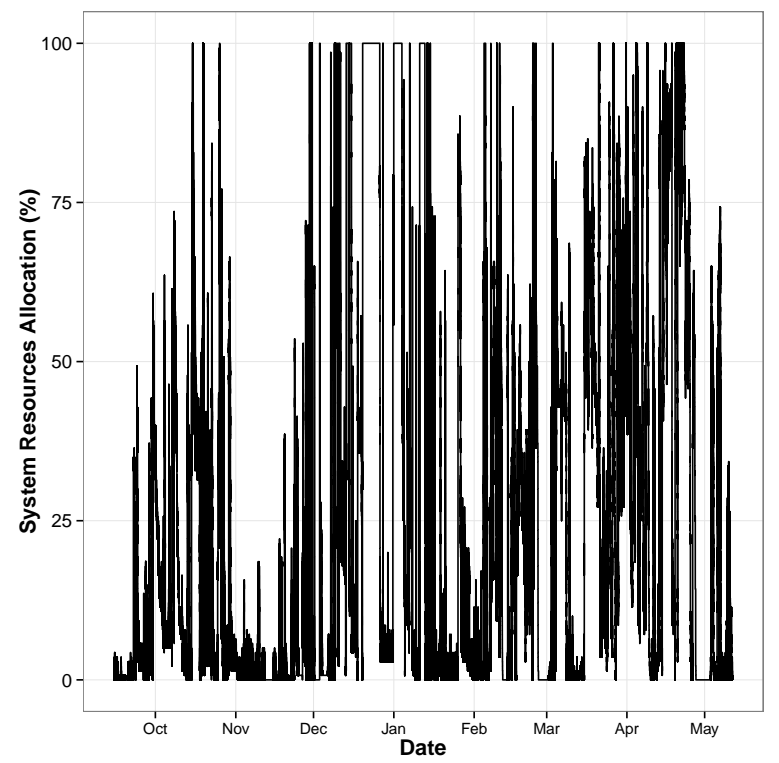

Figure 5.8: System resources utilization for the whole trace (OStrich)

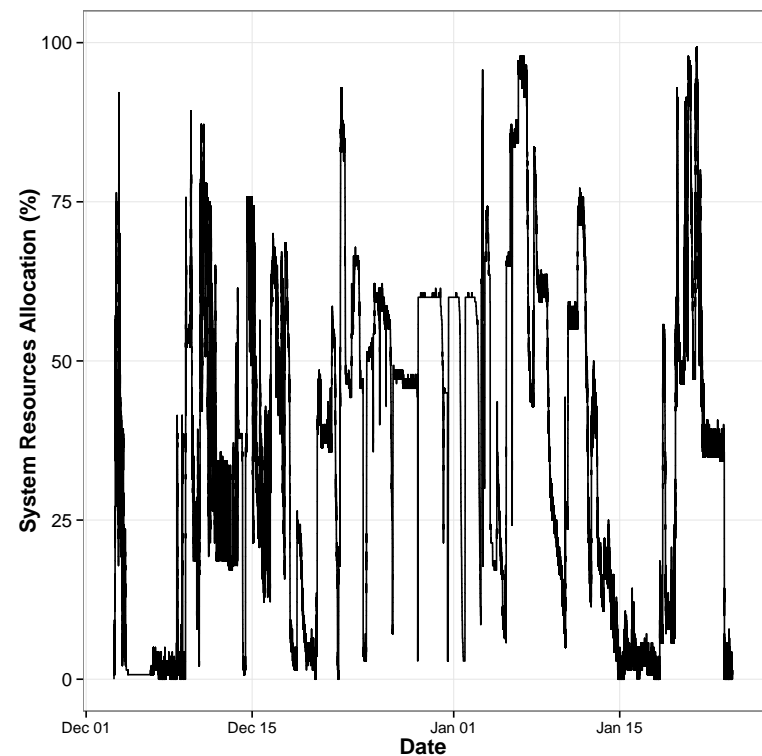

Figure 5.9: System resources utilization for the extract (original log: Maui, FCFS)

to the arrival of jobs earlier in the campaigns. As the no-dependency approach does not guarantee all the jobs precedence constraints from the original log, all the jobs can start at the beginning of the campaign. Thus the workload is not really the same. Due to this, we recommend the dependencies detection approach when making performance comparison between the replay output and the original log. It guarantees that user behavior and system 


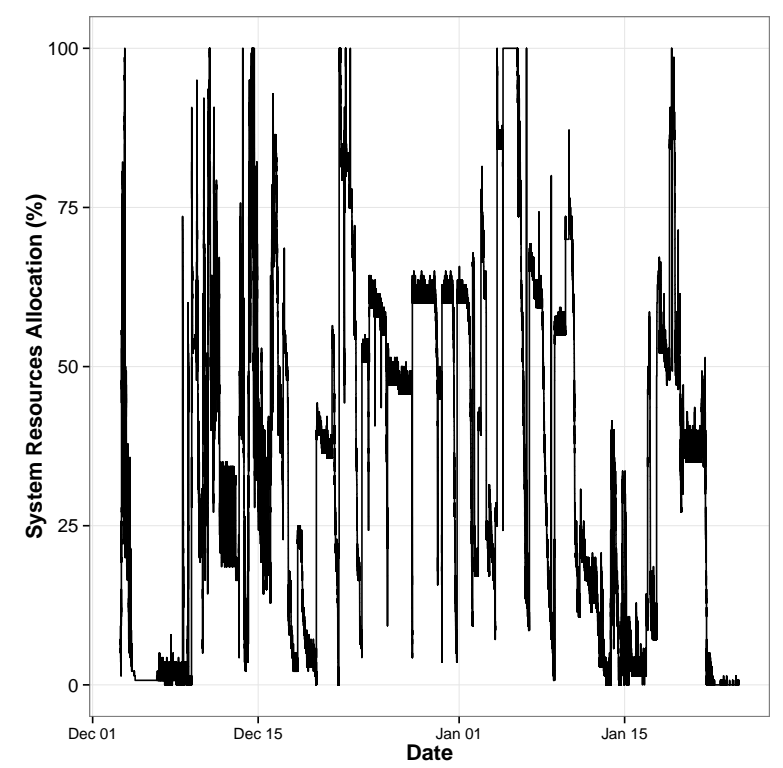

Figure 5.10: System resources utilization for the extract (OStrich, with job dependencies)

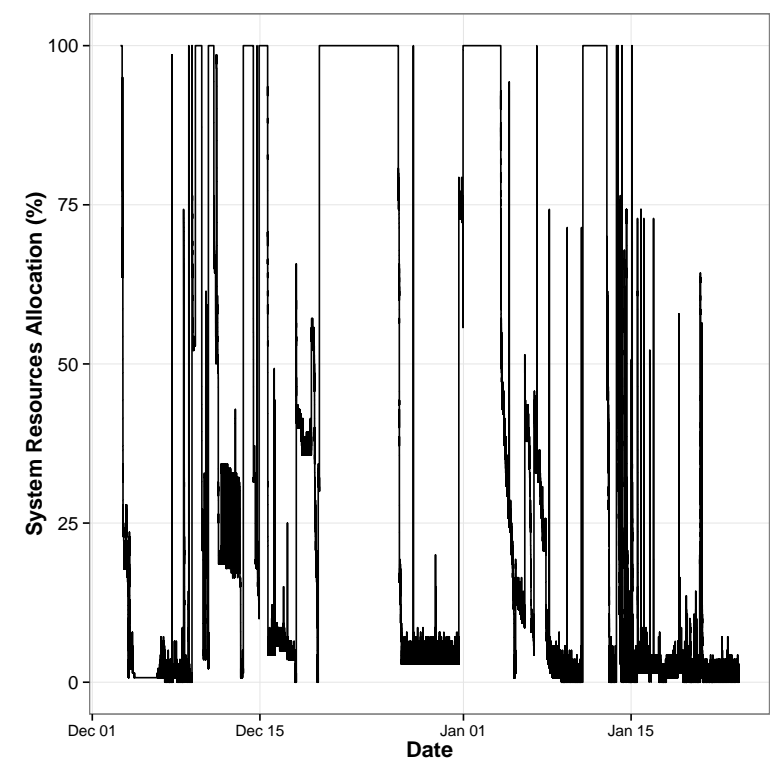

Figure 5.11: System resources utilization for the extract (OStrich, no dependencies)

utilization are consistent with the original output.

Now, we present another simulation to demonstrates how OStrich and FCFS react to different user profiles, concerning the job length that is preferably submitted by each profile. The simulator plays the role of a centralized scheduler: it takes an instance of a user workload a input, and it calculates the campaign stretches and the max-stretch per user 


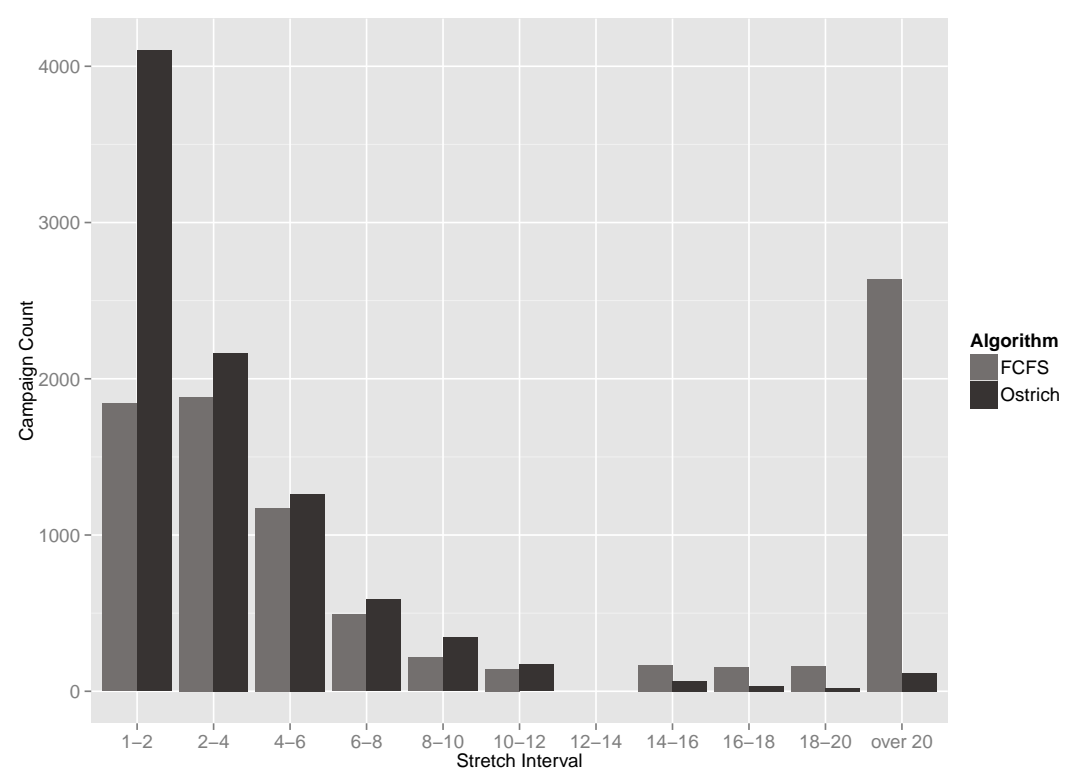

Figure 5.12: Ostrich vs FCFS: stretch values by intervals

obtained by each algorithm in an environment composed of $m=64$ identical processors. Due to the difficulty to find a real log with well defined user profiles, and in order to provide a better control of the experiment, we use a synthetic workload trace, generated to model two different user profiles.

We run 40 instances where instance is composed of $10^{4}$ jobs. For each job we set its length $p$ according to the user profile. We defined 2 user profiles: short-job profile and long-job profile. Short-job users submit short jobs with lengths uniformly taken from the range $\left[1 ; 3.6 \times 10^{3}\right]$ (seconds as time unit). Long-job users submit long jobs with lengths uniformly taken from the range $\left[3.6 \times 10^{3} ; 3.6 \times 10^{4}\right]$. Each job starts a new campaign with probability of 0.02 ; otherwise, it belongs to the previous campaign. If the job starts a new campaign, we set the owner of this campaign according to a uniform distribution.

In general, the results confirm our expectations and show that OStrich results in significantly lower max stretches than FCFS. The Figure 5.12 shows the distribution of stretch values for all campaigns. The number of campaigns with stretch lower than 2 for OStrich is more than twice the number obtained with FCFS. More important, though is the number of high stretch values above: while on OStrich this number decreases rapidly towards 0 as stretch increases, with FCFS it is bigger than 2600 above 20, representing 


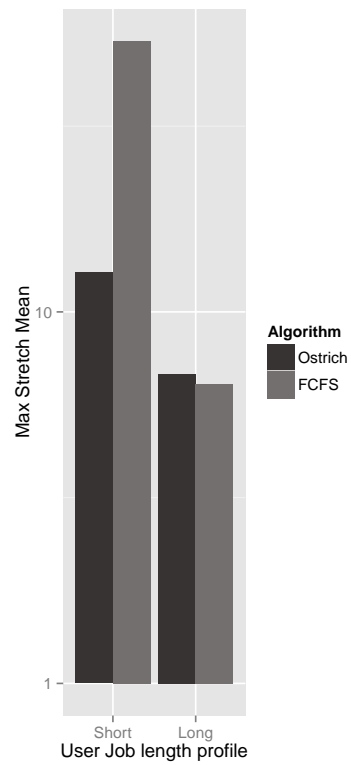

Figure 5.13: Ostrich vs FCFS: max campaign stretch mean per user profile

$42.3 \%$ of the total. The occurrence of stretch values above 20 is only 117 for OStrich (1.3\%).

The Figure 5.13 shows the max stretch average per user profile (in a log scale) and here we can see how OStrich accomplishes its purpose: short users are penalized by FCFS with big stretch values (whose average is above 50 ) while OStrich does not heavily discriminate users by their profiles, guaranteeing a more fair treatment for all the users (average of 12.8 for short users). For long users, FCFS and OStrich have almost the same performance (average of 6.3 for FCFS and 6.8 for OStrich).

In the next chapter, the OStrich is extended in order to handle parallel jobs. This extension manages the virtual schedule in a different way than when scheduling sequential jobs. The virtual schedule is modified like a malleable job, taking only the number of used processors to avoid priority inconsistencies. 


\section{Chapter 6}

\section{Scheduling parallel jobs with}

\section{OStrich}

The study of sequential job scheduling is useful for optimizing the execution of embarrassingly parallel applications. These applications follow the Bag-of-Tasks (BoT) model [LSV06, CM10], with no communication requirements. However, despite the applicability of this model, many scientific applications are composed of parallel jobs that need to be executed concurrently in order to synchronize the communication between their tasks and improve their performance.

In this chapter, the OStrich algorithm presented before is adapted to handle parallel jobs of type rigid. The theoretical analysis is updated accordingly and it shows that user performance is still bounded by his/her own workload and the number of active users. On the other hand, in this new setting the stretch also depends on the maximum utilization rate of the system (i.e. the maximum number of machines a parallel job is allowed to require).

\subsection{Parallel OStrich}

When dealing with parallel jobs, OStrich behavior is slightly different from the sequential job version. Now, the campaigns are executed following a greedy approach that strives to 


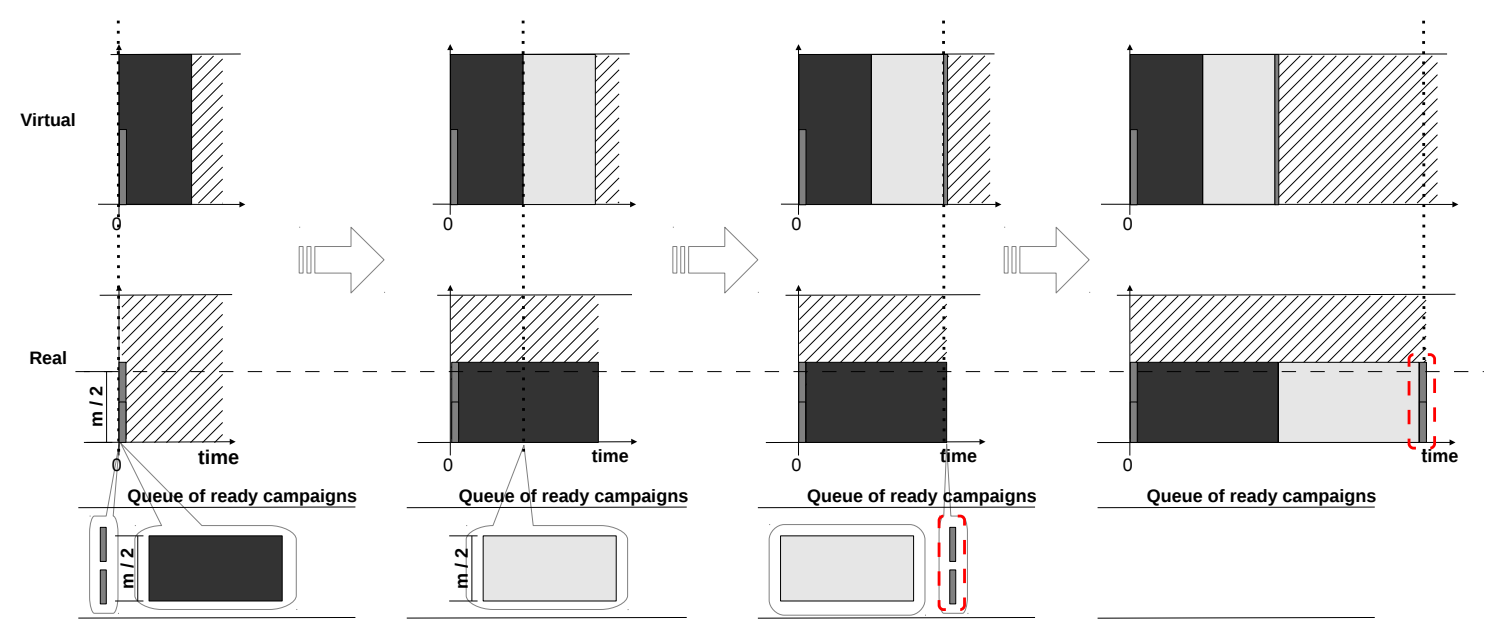

Figure 6.1: Delay problem between real and virtual schedule

keep all the machines occupied. The virtual and real schedule roles remain unaltered: the virtual schedule builds an ideal fair allocation of resources from which the priorities of the campaigns are derived and it is updated in an event driven fashion. The real schedule determines how the jobs are scheduled according to these priorities. But there are some changes concerned to the way the virtual schedule is build.

\subsubsection{Event based resizing}

In parallel job scheduling, it can not be guaranteed that all the machines will be occupied. Because of that, the delay between the real and virtual schedule is not bounded by a fixed amount as it was for sequential jobs. (in Chapter 5 it was proved that, for sequential jobs, the delay was bounded by a fixed amount). This can lead to a situation where campaigns start to be executed after their virtual completion times, resulting in wrong priority assignments.

In the Figure 6.1, an example with 3 users is given to illustrate how this might occur when the sequential version of OStrich is used to schedule parallel jobs. This example shows the evolution of the real and the virtual schedule in a system with $m$ identical processors. The users are identified by different shades of gray (dark, medium, and light gray). A dotted line is used to represent the present moment during the schedule, the dashed area represents idle processor time and a dashed line is used on the real schedule to split the 


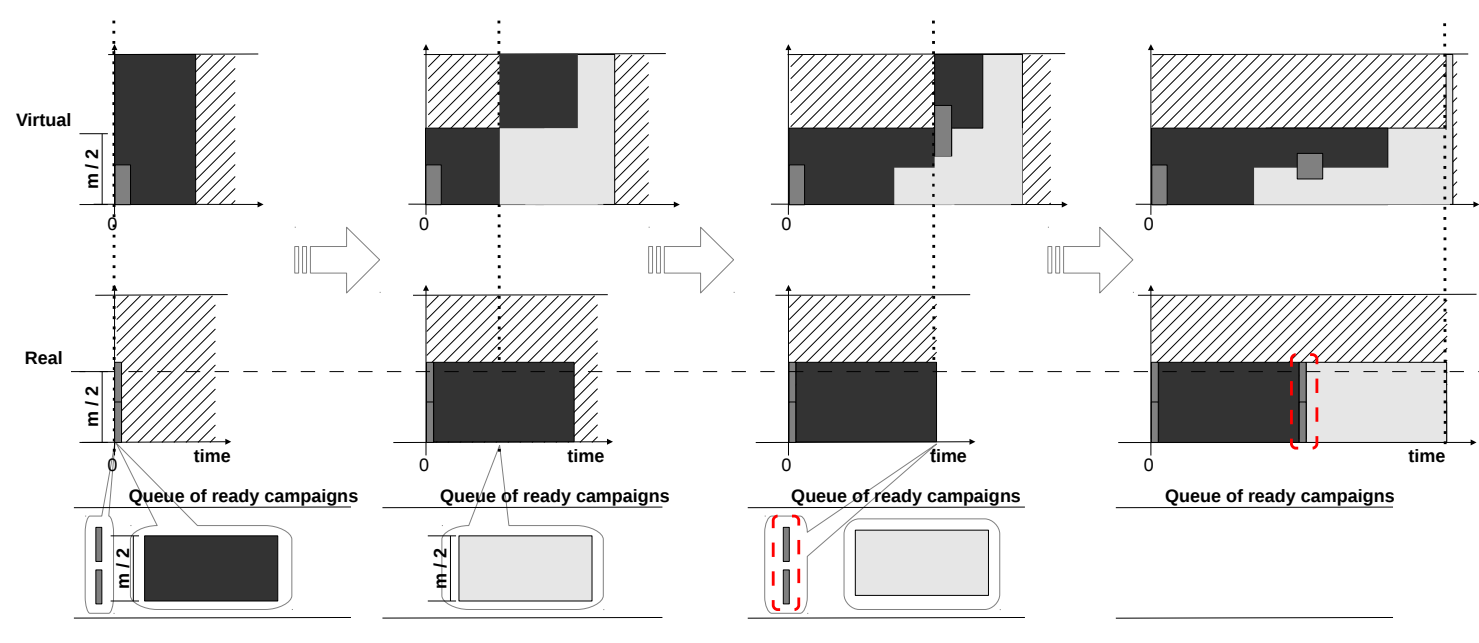

Figure 6.2: Delay correction using a resized virtual schedule

resources in two equal sets.

This example shows that the virtual schedule is computed using all the machines, regardless of the number of idle processors in the real schedule. The first two campaigns (from gray and dark gray users) are scheduled correctly. The third campaign (from the light gray user) arrives just after the virtual completion time of the dark gray user is achieved, so the higher priority remains with the dark gray user until his/her campaign finishes. However, the second campaign of the gray user arrives before the virtual completion time of the light gray user. The problem is that, despite having a very small workload, the campaign of the gray user must wait for the execution of the light gray campaign that has a higher priority and is still in the queue of ready campaigns. The conclusion is that using all the resources to compute the virtual schedule favors users that use less resources in situations of low utilization.

To tackle this issue, the virtual schedule must be resized to take into account the amount of idle processors on the real schedule. Figure 6.2 shows a corrected version of the same example, according to a resized virtual schedule.

More precisely, when an event occurs, the algorithm counts the number of idle processors between the present moment and the moment of the previous event. Then, it reduces the number of available processors in the virtual schedule during this time interval to be the same as in the real schedule. Then, the completion times are recomputed accordingly. 


\subsection{Algorithm description}

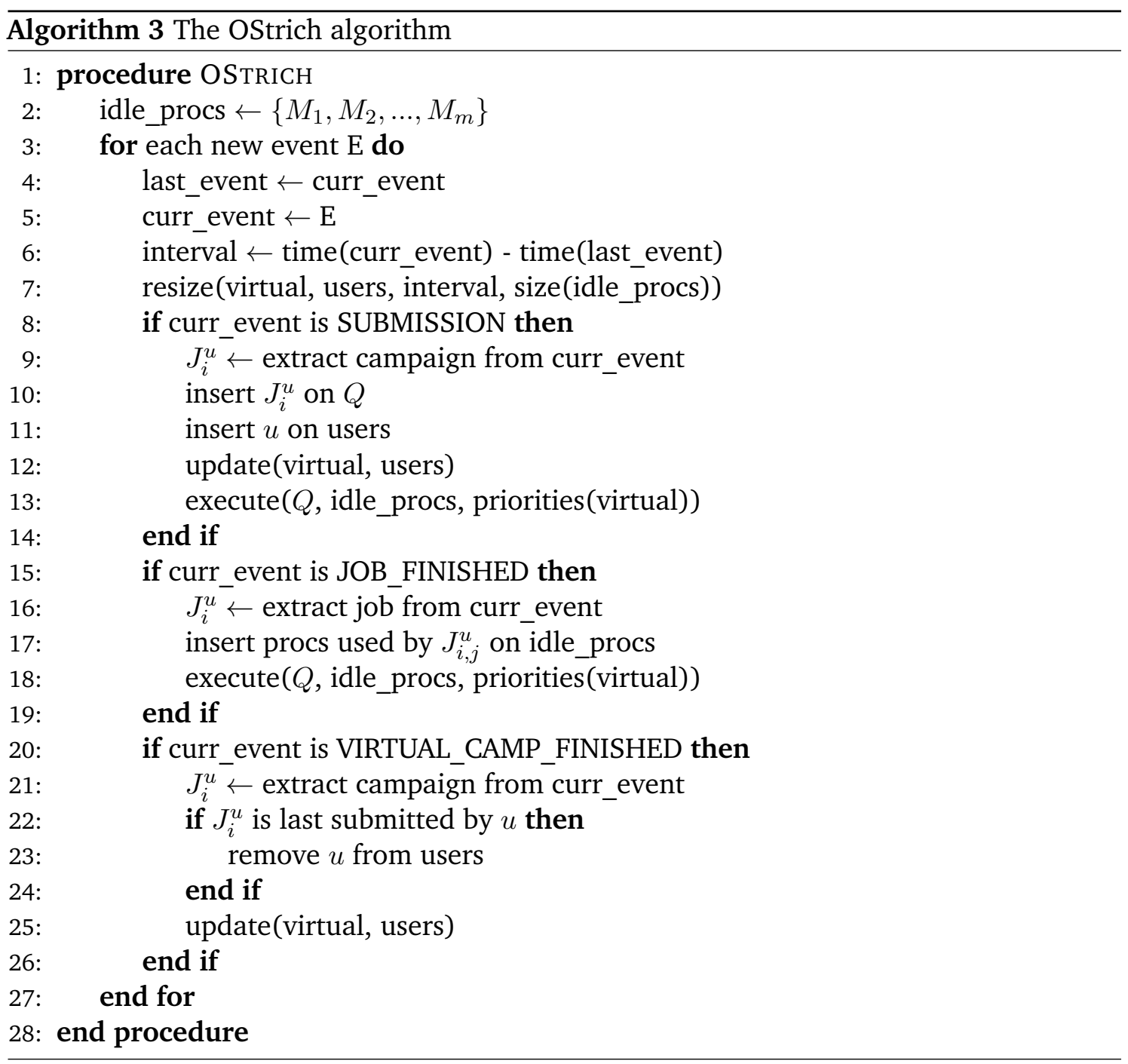

First, in the beginning of the scheduler, the set of idle processors contains all the machines, as none of the resources are being used (line 2).

Inside the for loop, the algorithm waits for the next event (line 3) and when it happens, the virtual schedule is resized to not take into account the resources that were idle between the present event and the previous one (line 7). This resize function is also responsible for recomputing the campaigns virtual completion times. The three types of events that are handled by the algorithm are campaign submissions (line 8), job completions (line 15) and virtual completion time of campaigns (line 20).

When a campaign is submitted, it is put on the list of ready jobs represented by $Q$ 
(line 10) and the user is added to the set of active users (line 11). The virtual schedule is updated to reset the virtual completion times and the scheduler proceeds the execution with an asynchronous call (lines 12 and 13). That way, after the call, the execution flow goes back and waits for another event to happen.

The execute function called in lines 13 and 18 executes the campaigns according to the following greedy algorithm:

1. Look at the current amount of machines available;

2. Find the first job in the queue of ready campaigns that fits on these available machines and schedule it on these machines;

3. Remove these machines from the set of idle processors;

4. Repeat this process until there are no more jobs to schedule.

Inside the queue, the jobs of the campaigns are ordered according to LJF (Largest-JobFirst) [LC91], that is, according to job's size $\left(q_{i, j}^{u}\right)$ in non-increasing order. This technique has been proven to lead to higher utilization than SJF (Shortest-Job-First) and FCFS [Aid00, HL10].

When a job finishes, the processors that were allocated to it are freed (line 2) and the scheduler proceeds the execution in the same manner as in the paragraph above.

Finally, when the virtual completion time of a campaign is reached, its owner is removed from the list of active users and the virtual schedule is updated (line 23). But this happens only if this campaign is the last one submitted by the user. Otherwise, the user will remain active, given that this campaign will be followed by another campaign.

\subsection{Example}

The Figure 6.3 shows the evolution of the real and the virtual schedules generated by OStrich from $t=0$ to $t=7$ in a system with 5 identical processors. This example shows 3 submissions issued from 3 different users identified by shades of gray (dark, medium and light gray). The size and the length of each job is indicated in a label with 

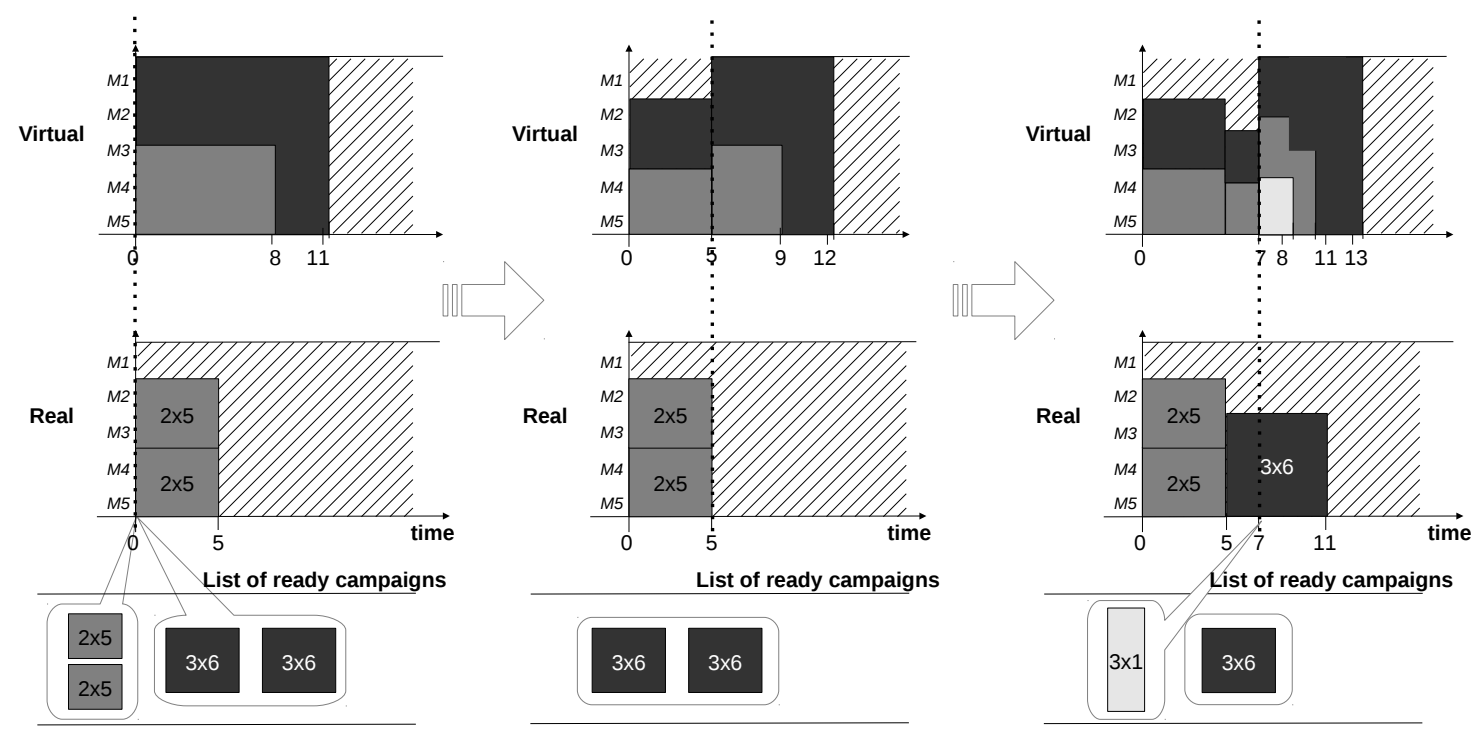

Figure 6.3: Virtual and real schedule generated by the OStrich algorithm with 3 users

$\mathrm{a}<$ size $>\mathrm{x}<$ length $>$ format. A dotted line is used to represent the present moment during the schedule and the dashed area represents idle processor time.

At time $t=0$, two campaigns from distinct users are submitted. At this point, the virtual schedule is constructed taking into account all the machines and the medium gray user has the higher priority. At time $t=5$ the first jobs finish and, following this event, the virtual schedule is resized to take into account only the number of used processors. Since there are no more jobs from the medium gray user, the dark gray user is the next on the priority list and its first job is selected for execution. Finally, at $t=7$, the light gray user submits its first campaign. Following this new submission, the virtual schedule is updated onward and the light gray user gets the higher priority. However, note that he/she will have to wait until $t=11$, when the executing dark gray job finishes. Still at this moment, the virtual schedule is resized again, taking the number of used processors since the last event.

\subsection{Comparison with existing scheduling strategies}

In this section, we use an example with parallel jobs to compare OStrich with space sharing and FCFS (First-Come-First-Served). The purpose is to show how OStrich can offer a solution that, while following a space sharing strategy, makes a more wise use of the 


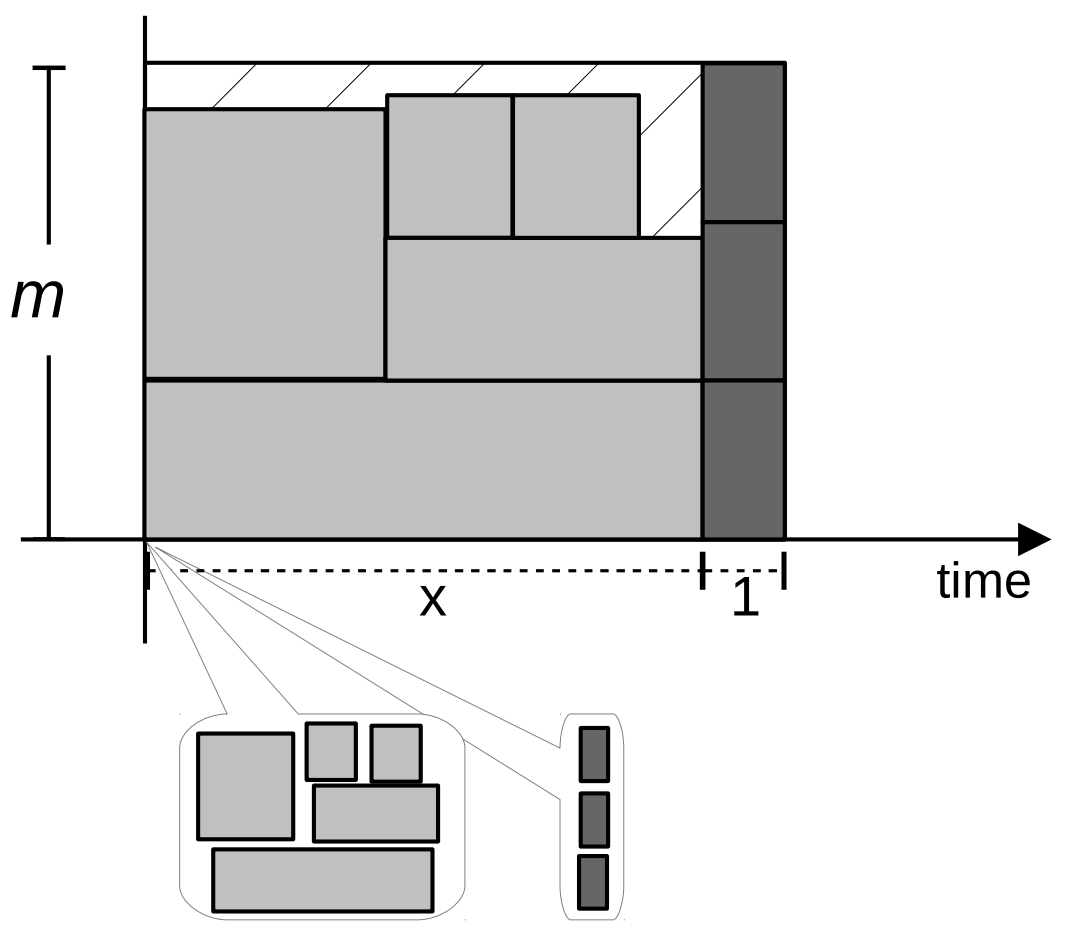

Figure 6.4: FCFS example with 2 users

system through a time sharing based execution that improves the stretch experience of users.

In this example, the loads are 2 campaigns, each one issued by a different user, submitted at the same moment on the beginning of the schedule. The users are represented by two shades of gray and the optimal lengths for the campaigns are $x$ and 1 for the light gray and dark gray user, respectively.

FCFS executes jobs according to their order of arrival. It is a classical and widely used strategy, because it is very simple to understand and to implement, and jobs do not starve. However, it is well known that, for stretch optimization, FCFS can be arbitrarily far from the optimal as illustrated in Figure 6.4. In this figure, the light gray campaign obtains an optimal stretch. But the dark gray campaign obtains a stretch of $x$, that corresponds to the length of the light gray campaign. Stretch, in this case, depends on the load submitted by other users.

Space share is a common strategy used by actual systems to provide fairness between users. In fairshare the users are given the same share of resources. However, this results in 


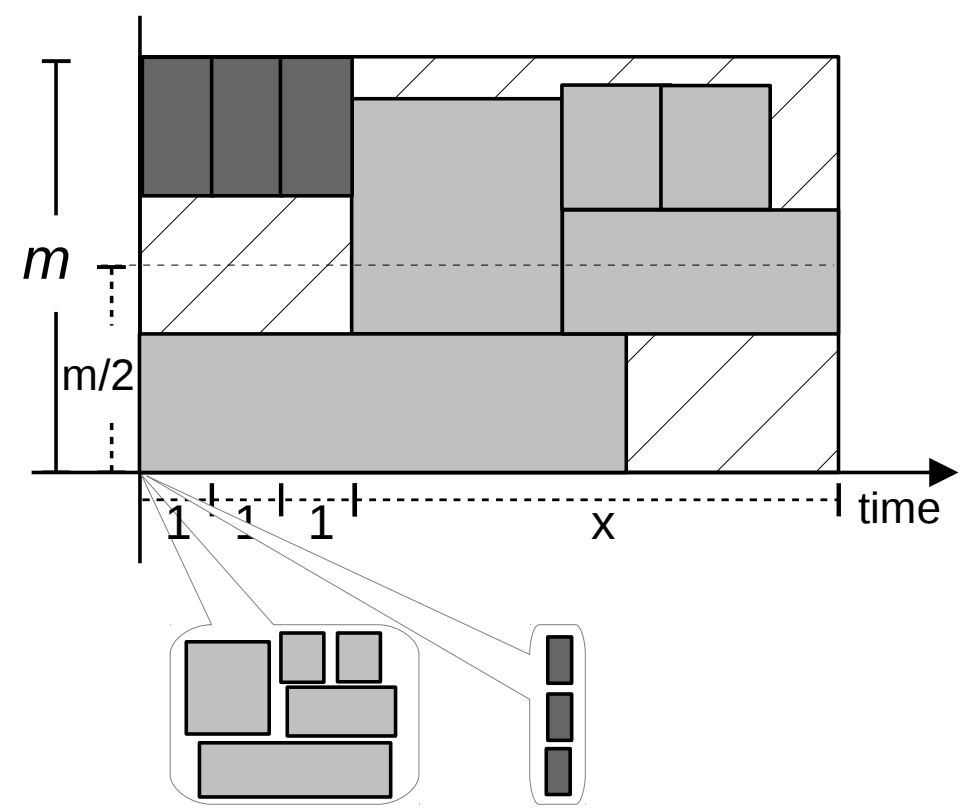

Figure 6.5: Space share example with 2 users

a very poor performance for the users since they can only use just the amount of resources relative to their shares. Therefore, this strategy inherently strives to give a stretch of $k$ for all the users (where $k$ is the number of active users). Another disadvantage is that, large jobs cannot be executed if their sizes exceed the owner share. They would wait until the user quota increases, which is not guaranteed to happen.

Figure 6.5 shows the same situation as before, now with campaigns being scheduled by the fairshare algorithm. We can see that, from the beginning of the schedule, the resources are divided by 2 and the first jobs are scheduled within these shares. The gray user jobs are executed in sequence because there is not enough share to execute them in parallel. This causes the dark gray campaign stretch to be 3 , with the light gray campaign stretch being $x+3$.

OStrich also provides fairness between users but it uses the fairshare strategy just to give priorities to the user campaigns, leaving the execution in charge of a greedy algorithm. The users with the higher priority have their jobs executed first and can use all the machines.

Figure 6.6 shows how the same input of the earlier examples are handled by OStrich. In this case, along with the real schedule there is a virtual fairshare schedule that gives the higher priority to the dark gray campaign. It is executed first by the greedy algorithm, 


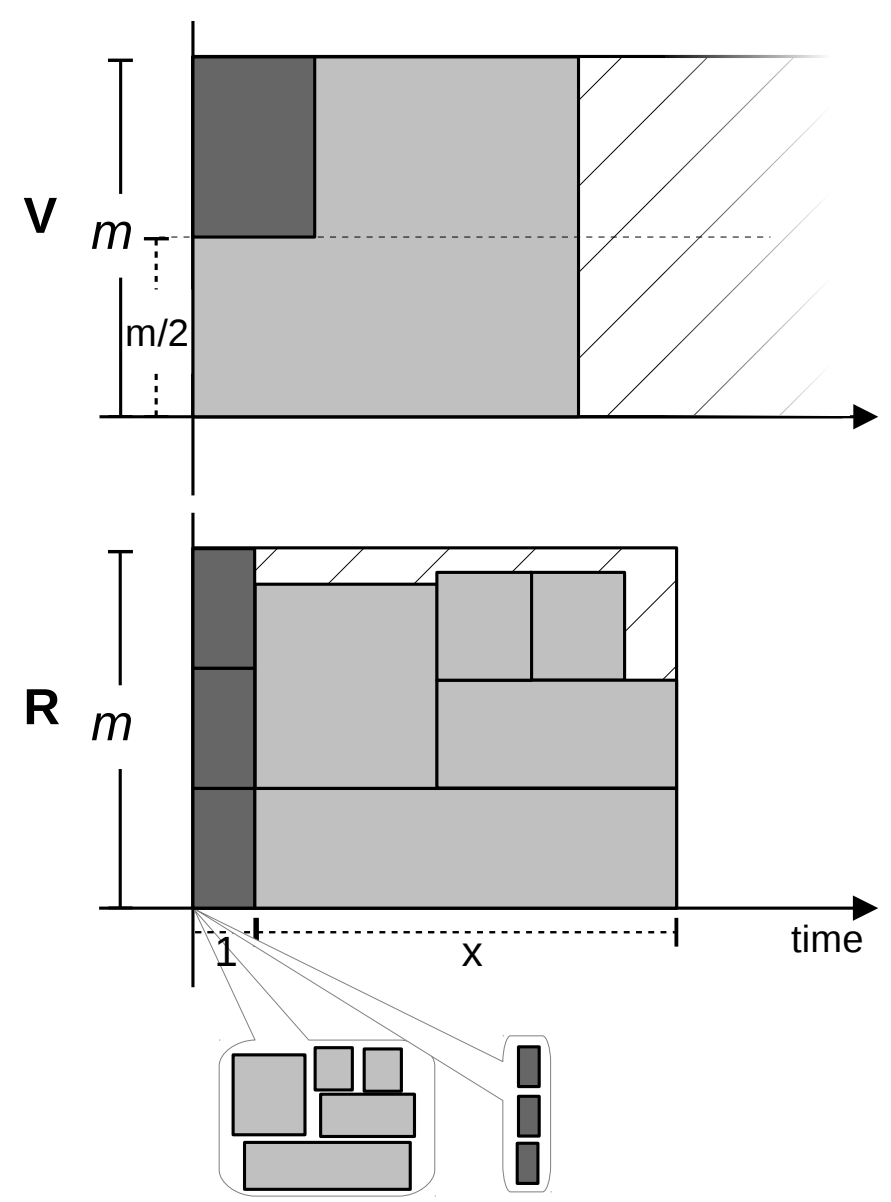

Figure 6.6: OStrich example with 2 users

followed by the light gray campaign. In this case, the dark gray campaign stretch is optimal. The light gray campaign stretch is $x+1$ and, although not optimal, is still better than the one obtained with fairshare.

\subsection{Theoretical analysis}

In this section we bound the worst case stretch of a campaign in Parallel OStrich. The idea of the main proof is to bound the completion time of the last job of a campaign using a "conservative space" argument between the real and the virtual schedule. The "space" is 
a measure in terms of amount of time $\times$ number of processors.

In the proofs outlined in this section, $p_{\max }$ denotes the maximum job length in the system and $\alpha$ is the maximum utilization rate allowed per job where $\alpha \in[0,1]$. So, $\alpha m$ is the maximum job size. The virtual schedule is denoted by $V$ and the real schedule by $R$. To simplify the formulation of proofs, we say that the virtual schedule $V$ "executes" jobs even though $V$ is just an abstraction used for defining the priority of real jobs. At time $t$, a job is "executed" by $V$ if in $V$ there is a fraction of processors assigned to this job.

\subsubsection{Worst-case bound}

First, we will prove that maximum number of idle processors in $R$ is a function of $\alpha$.

Lemma 6.1. At any time $t$, if $|Q| \neq \emptyset$, then at most $\alpha m$ processors are idle on $R$, where $Q$ is the queue of ready jobs.

Proof. Consider a schedule $S$ constructed by OStrich. Let us assume by contradiction that at some time $t$ the number of idle processors is bigger than $\alpha m$ and $|Q| \neq \emptyset$. Now, consider $J$ as the first job started after $t$. So, as $J$ has size at maximum $\alpha m$ and $J \in Q$ at moment $t$, then $J$ could be scheduled earlier at time $t$. So, the schedule $S$ was not constructed by a greedy algorithm. But this is a contradiction since OStrich is greedy.

Now, we prove that the idle space in $R$ is the same represented in $V$.

Lemma 6.2. The idle space on $V$ is equal to the idle space on $R$ for any time interval $\left[t_{i}, t_{i+1}\right]$.

Proof. In the real schedule, the number of idle processors can be changed only at two events. The first one is when a job is done. When this happens, the processors that were allocated to this job are freed and become idle. The second event is when a new campaign is submitted, causing idle processors to be allocated to new jobs. These two events are represented in the Algorithm 3. In this same algorithm, between any two events, the virtual schedule will always be adjusted, i.e., the same idle space present in $\mathrm{R}$, between the two events, will also be inserted to V. 
Corollary, from this lemma: since $V$ and $R$ have the same number of machines, the amount of space in $R$ used to execute jobs between a time interval $\left[t_{i}, t_{i+1}\right]$ is the same in $V$.

Lemma 6.3. The completion time $C_{i, q}^{u}$ of the last piece $q$ of a campaign $J_{i}^{u}$ is bounded by a sum:

$$
C_{i, q}^{u} \leq t_{i}^{u}+\frac{\frac{k W_{i-1}^{u}}{m}+\frac{(k-1) W_{i}^{u}}{m}+\frac{W_{i}^{u}}{m}}{(1-\alpha)+p_{\max }}
$$

Proof. For the first part of this proof, let us consider as if all the $m$ machines were used all the time during the schedule. The part $k W_{i-1}^{u} / m$ corresponds to the maximum time the previous campaign of the same user can take to execute and so, the campaign $J_{i}^{u}$ must wait this time at maximum to be able to start its execution. The part $(k-1) W_{i}^{u} / m$ expresses the maximum time that takes to execute all the campaigns with higher priorities than $J_{i}^{u}$. Finally, $W_{i}^{u} / m+p_{\max }$ bounds the maximum time that takes to execute $J_{i}^{u}$.

Using all the machines, the executed area between $\tilde{s}_{i}^{u}$ and $\tilde{C}_{i}^{u}$ is at most $\left(\tilde{C}_{i}^{u}-\tilde{s}_{i}^{u}\right) m$. However, according to Lemma 1 , the idle space between $\tilde{s}_{i}^{u}$ and $\tilde{C}_{i}^{u}$ can be at most $\left(\tilde{C}_{i}^{u}-\tilde{s}_{i}^{u}\right) \alpha m$ and, so, the executed area can be $\left(\tilde{C}_{i}^{u}-\tilde{s}_{i}^{u}\right)(1-\alpha) m$ in the worst case. Hence, the stretch of any campaign within the executed area will be at most $\left(\left(\tilde{C}_{i}^{u}-\right.\right.$ $\left.\left.\tilde{s}_{i}^{u}\right) m\right) /\left(\left(\tilde{C}_{i}^{u}-\tilde{s}_{i}^{u}\right)(1-\alpha) m\right)=1 /(1-\alpha)$.

Finally, regarding $p_{\max }$, the campaign can finish in the real schedule after its completion on the virtual schedule $\left(\tilde{C}_{i}^{u}\right)$. However, according to Lemma 2 , the idle (and executed) spaces of $R$ and $V$ are equal for the same time interval. From this, we conclude that if the last part of $J_{i}^{u}$ is beyond $\tilde{C}_{i}^{u}$ by an exceeding area $S$, it means that an equal area $S^{\prime}$ composed of lower priority jobs were executed before $\tilde{C}_{i}^{u}$. But the interval $C_{i}^{u}-\tilde{C}_{i}^{u}$ can not be bigger than $p_{\max }$, otherwise one or more jobs from $J_{i}^{u}$ would have to be started in $S$, even having a higher priority than the ones represented by $S^{\prime}$. But this is a contradiction.

The Figure 6.7 illustrates the notations used in this proof and can be used to facilitate the comprehension. 


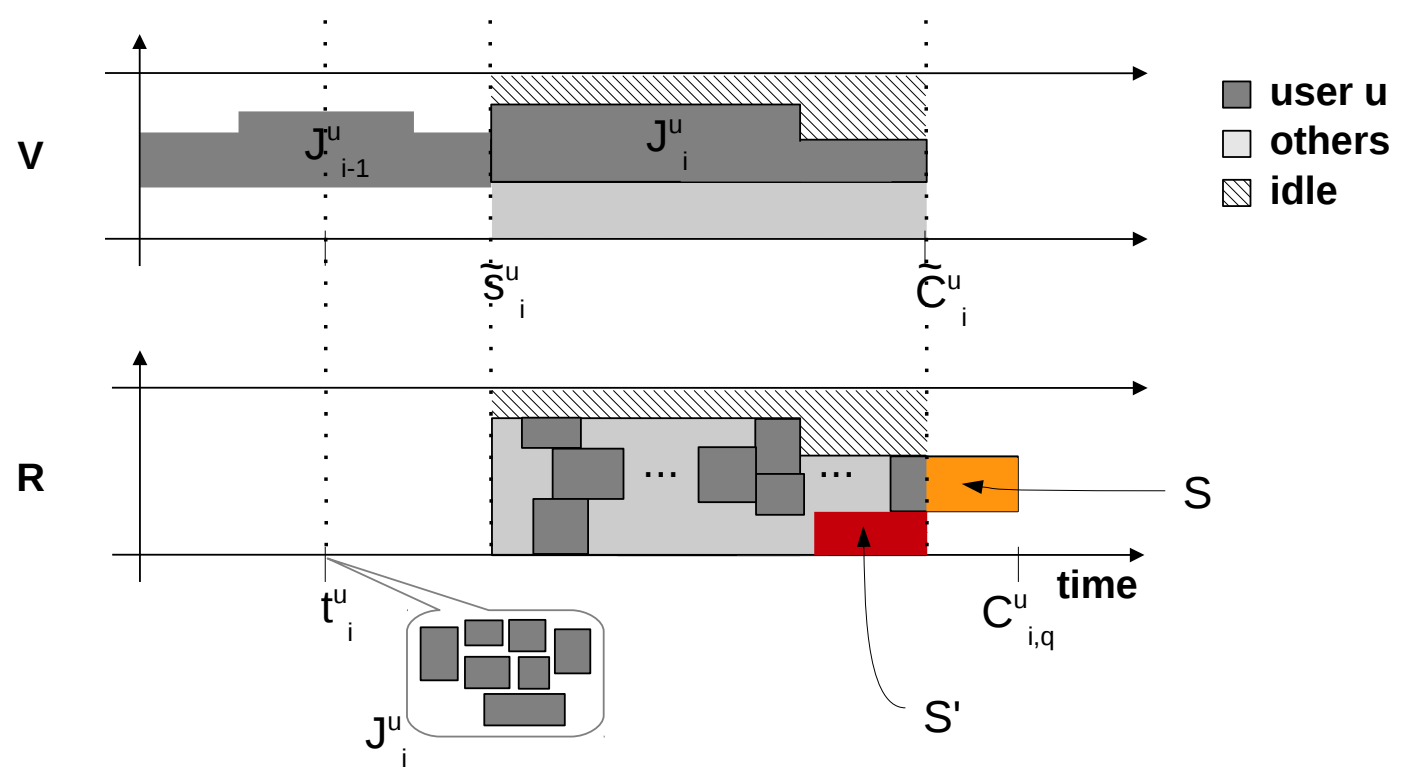

Figure 6.7: Analysis of OStrich with parallel jobs: stretch bound of a campaign execution

The Equation 6.1 can be simplified to:

$$
C_{i, q}^{u} \leq t_{i}^{u}+\frac{k\left(W_{i-1}^{u}+W_{i}^{u}\right)}{m(1-\alpha)}+p_{\max }
$$

Theorem 6.1. The stretch of a campaign is proportional to the number of active users $k$ and the maximum utilization rate $\alpha$. More formally, $D_{i}^{u} \in O\left(\frac{k}{1-\alpha}\left(\frac{W_{i-1}^{u}}{W_{i}^{u}}\right)+1\right)$

Proof. The result follows directly from Lemma 6.3 and from the definition of campaign stretch (Section 3.3). Recall that, for campaign $J_{i}^{u}$, the stretch $D_{i}^{u}$ is defined by $D_{i}^{u}=$ $\left(C_{i}^{u}-t_{i}^{u}\right) / \max \left(W_{i}^{u} / m, p_{\max }\right)$. Also, $C_{i}^{u}=C_{i, q}^{u}$, i.e. the completion time of a campaign is equal to the completion time of its last "piece". Replacing $C_{i}^{u}$ by the definition of $C_{i, q}^{u}$ in Equation 6.2, we have:

$$
D_{i}^{u} \leq \frac{\frac{k\left(W_{i-1}^{u}+W_{i}^{u}\right)}{m(1-\alpha)}+p_{\max }}{\max \left(\frac{W_{i}^{u}}{m}, p_{\max }\right)} \leq \frac{k}{1-\alpha}\left(\frac{W_{i-1}^{u}}{W_{i}^{u}}+1\right)+m \cdot p_{\max }
$$

For a given parallel system, $m$ is constant. Similarly, the maximum size of a job $p_{\max }$ can be treated as constant, as it is typically limited by system administrators. Hence, $D_{i}^{u} \in O\left(\frac{k}{1-\alpha}\left(\frac{W_{i-1}^{u}}{W_{i}^{u}}\right)+1\right)$. 


\section{Chapter 7}

\section{Conclusion and ongoing work}

The popularization of parallel systems leveraged by the emergence of low cost desktop platforms like clusters, grids and, more recently, clouds promotes the emergence of new user profiles and applications, whose needs impose new challenges for the HPC community, particularly on the field of scheduling theory. In the last decades, the fair division of resources has attracted the attention of scientists from many areas such as mathematics, physics, economics and computer science. Nevertheless, existing theoretical models did not capture all the dynamics involved in real environments.

In this thesis, we study fairness in multi-user parallel systems over different settings to provide solutions where all users feel satisfied about the way resources are shared among them. In order to model submissions issued by many users over time, we define and use the Campaign model where each user submits campaigns of jobs in an interactive manner. We provide an algorithm that schedules the resources among users in a fair way without compromising system and individual performances. Among all the techniques available to achieve fairness, the stretch metric was chosen because it measures the responsiveness of the system taking into account each user expectation.

The solutions presented in this thesis propose a compromise between fairness and individual performance for different Campaign Scheduling scenarios. These solutions are implemented as online scheduling algorithms that explicitly maintain fairness among users by bounding the worst-case stretch of each campaign whereas the stretch is defined by 
means of a function derived from the campaign's response time. It is shown that the performance experienced by a user does not depend on the total load of the system, but only on his/her submissions' loads and on the number of users competing for the processors. Furthermore, in some cases, the performance of each user can be optimized.

More specifically, we can conclude that:

- it is possible to maintain fairness among users in an online fashion, with bounded performance. The solutions proposed in this thesis, specially OStrich and parallel OStrich, are suitable fair solutions to be considered since they deliver theoretical performances that are better than actual fair schedulers .

- According to the analysis and simulations presented throughout this thesis, fair scheduling is necessary in order to provide responsiveness, specially in heavy loaded systems, on which the discrepancy between the solutions proposed and actual solutions achieve is bigger.

OStrich works with the premise that job processing times are known in advance as opposite to the majority of actual system schedulers. In practice, even when processing time estimates are requested from the user, the real processing times can be radically different. In fact, most actual systems as Slurm [YJG03] and OAR [CDCG ${ }^{+}$05] handle this issue by punishing users who make bad predictions that causes misuse of resources. OStrich can also be adapted to handle user punishment. As an example, the ratio between predicted processing time and real processing time could be used to adjust the user share in the virtual schedule causing changes in the priorities. In this thesis, we chose to not tackle this subject because it is a complementary mechanism. It can be used to adjust the priorities but it is not relevant as the fairness per se.

\subsection{Work contributions and perspectives}

This thesis contributes in both theoretical and practical sides. From the theoretical pointof-view, it presents algorithms that allows a fair scheduling in a multi-user parallel system under realistic assumptions, along with models, definitions, lemmas, theorems and proofs 
that sustain these capabilities. From the practical point-of-view, it provides a campaign scheduling simulator and the results provided by it. The simulator code is available at https: //forge.imag.fr/frs/download.php/568/cssim.zip, but before downloading, an account must be created at https://forge.imag.fr/account/register.php.

These contributions were organized and published at international events. In December 2012, the campaign model and the FairCamp algorithm were published in the annual IEEE International Conference on High Performance Computing (HiPC 2012), held in Pune, India. In September 2013, the OStrich algorithm was published in the 10th International Conference on Parallel Processing and Applied Mathematics (PPAM 2013), held in Warsaw, Poland.

Below, the publications with more detailed information.

- Campaign Scheduling. Vinicius Pinheiro, Krzysztof Rzadca and Denis Trystram. The IEEE International Conference on High Performance Computing (HiPC), pages 1-10. Pune, India, December 2012.

- OStrich: Fair Scheduling For Multiple Submissions. Joseph Emeras, Vinicius Pinheiro, Krzysztof Rzadca and Denis Trystram. The International Conference on Parallel Processing and Applied Mathematics (PPAM 2013). LNCS. Springer, 2013.

- Fair Scheduling for Multiple Submissions. Joseph Emeras, Vinicius Pinheiro, Krzysztof Rzadca and Denis Trystram. Tech Report. Université de Grenoble, June 2012.

I also co-authored a paper along with professors Krzysztof Rzadca and Denis Trystram on the 10th conference on "New Challenges in Scheduling Theory", held in Frejus, France, in October 2012. The paper was entitled "Fairness Between Users in the Campaign Scheduling Problem".

Still, focusing on Parallel OStrich, we submitted a paper with the most recent results entitled "Online Fair Scheduling of Burst Submissions of Parallel Jobs" to the 27th IEEE International Parallel \& Distributed Processing Symposium (IPDPS 2014) that will be held on May 2014 in Phoenix, USA. 
As future directions, we plan to analyze the Campaign Scheduling problem and the solutions provided in this thesis under the light of game theory. More specifically, we plan to study the envy-freeness property and if it can be guaranteed by the algorithms that were proposed in this work.

Further, we plan to relax the campaign scheduling model to be more general, including campaign execution overlapping. We also plan to analyze the behavior of OStrich in a general multi-user scheduling problem, with users submitting single and independent jobs instead of whole campaigns and the analysis being towards the max-stretch of individual jobs.

In the practical side, we plan to do experiments with OStrich in a production system with real users and compare it to SLURM. In order to perform this, the first step is already done given that an implementation of OStrich is recently available at https://github.com/ filipjs/OStrich, as a plugin for the SLURM resource manager .

\section{Special acknowledgment}

This work was supported by the French-Polish bilateral cooperation program "Polonium" and by the CAPES/COFECUB program (project number 4971/11-6). The PhD candidate held part of his studies at University of Grenoble, from October 2011 to September 2012, under the terms of a co-supervision scholarship between the University of São Paulo and the University of Grenoble. 


\section{Bibliography}

[Ada65] J Stacy Adams. Inequity in social exchange. Advances in experimental social psychology, 2(267-299), 1965. 3

[Aid00] Kento Aida. Effect of job size characteristics on job scheduling performance. In DrorG. Feitelson e Larry Rudolph, editors, Job Scheduling Strategies for Parallel Processing, volume 1911 of Lecture Notes in Computer Science, pages 1-17. Springer Berlin Heidelberg, 2000. 83

[AMPP04] Alessandro Agnetis, Pitu B. Mirchandani, Dario Pacciarelli e Andrea Pacifici. Scheduling problems with two competing agents. Operations Research, 52(2):229-242, 2004. 17

[And04] D. P. Anderson. Boinc: a system for public-resource computing and storage. In Grid Computing, 2004. Proceedings. Fifth IEEE/ACM International Workshop on, pages $4-10$, nov. 2004. 2, 33

[BCM98] Michael A. Bender, Soumen Chakrabarti e S. Muthukrishnan. Flow and stretch metrics for scheduling continuous job streams. In Proceedings of the ninth annual ACM-SIAM symposium on Discrete algorithms, SODA '98, pages 270-279, Philadelphia, PA, USA, 1998. Society for Industrial and Applied Mathematics. 24, 37, 44

[BCS74] J. Bruno, Jr. E. G. Coffman e R. Sethi. Scheduling independent tasks to reduce mean finishing time. Commun. ACM, 17(7):382-387, July 1974. 15, 25, 58

[BK] Peter Brucker e Sigrid Knust. Complexity results for scheduling problems. 
http://www.informatik.uni-osnabrueck.de/knust/class/. Last accessed on 19 Sep, 2012. 2, 11, 12

[BMR02] Michael A. Bender, S. Muthukrishnan e Rajmohan Rajaraman. Improved algorithms for stretch scheduling. In Proceedings of the thirteenth annual ACM-SIAM symposium on Discrete algorithms, SODA '02, pages 762-771, Philadelphia, PA, USA, 2002. Society for Industrial and Applied Mathematics. 24

[BTEP00] Jacek Blazewicz, Denis Trystram, Klaus Ecker e Brigitte Plateau. Handbook on parallel and distributed processing. Springer-Verlag New York, Inc., 2000. 1, 10

$\left[\mathrm{CDCG}^{+}\right.$05] Nicolas Capit, Georges Da Costa, Yiannis Georgiou, Guillaume Huard, Cyrille Martin, Grégory Mounié, Pierre Neyron e Olivier Richard. A batch scheduler with high level components. In Cluster Computing and the Grid, 2005. CCGrid 2005. IEEE International Symposium on, volume 2, pages 776-783. IEEE, 2005. 26,92

[CDS10] H. Casanova, F. Desprez e F. Suter. Minimizing stretch and makespan of multiple parallel task graphs via malleable allocations. In Parallel Processing (ICPP), 2010 39th International Conference on, pages 71 -80, sept. 2010. 24

[CM10] Javier Celaya e Loris Marchal. A fair decentralized scheduler for bag-of-tasks applications on desktop grids. In Cluster, Cloud and Grid Computing (CCGrid), 2010 10th IEEE/ACM International Conference on, pages 538 -541, may 2010. $21,24,79$

[DLG11] Bruno Donassolo, Arnaud Legrand e Cláudio Geyer. Non-cooperative scheduling considered harmful in collaborative volunteer computing environments. In Proceedings of the 2011 11th IEEE/ACM International Symposium on Cluster, Cloud and Grid Computing, CCGRID '11, pages 144-153, Washington, DC, USA, 2011. IEEE Computer Society. 3 
[Dro09] M. Drozdowski. Scheduling for Parallel Processing. Computer Communications and Networks. Springer, 2009. 21, 30

[ER06] Stephen Emmott e Stuart Rison. Towards 2020 science. Tech report, Working Group Reserach. Microsoft Research Cambridge, 2006. 1

[Fei] Dror G. Feitelson. Parallel workload archive. http://www.cs.huji.ac.il/labs/ parallel/workload/. Last accessed on 28 Sep, 2012. 71

[Fei05] Dror Feitelson. Workload Modeling for Computer Systems Performance Evaluation. 2005. 68

[Geo10] Yiannis Georgiou. Contributions for Resource and Job Management in High Performance Computing. PhD Thesis, Université de Grenoble, France, 2010. Supervisor - Olivier Richard; Co-supervisor - Jean François Méhaut. 27

[GLLRK79] R. L. Graham, E. L. Lawler, J. K. Lenstra e A. H. G. Rinnooy Kan. Optimization and approximation in deterministic sequencing and scheduling: a survey. Annals of discrete mathematics, 5(2):287-326, 1979. 11, 37

[Gra66] R. L. Graham. Bounds for certain multiprocessing anomalies. Bell System Technical Journal, 45:1563-1581, 1966. 12

[Gra69] R. L. Graham. Bounds on multiprocessing timing anomalies. SIAM JOURNAL ON APPLIED MATHEMATICS, 17(2):416-429, 1969. 14, 25, 58, 71

[GS58] G. Gamow e M. Stern. Puzzle-math. Macmillan, 1958. 4

[Hen95] Robert L Henderson. Job scheduling under the portable batch system. In Job scheduling strategies for parallel processing, pages 279-294. Springer, 1995. 26

[HL10] Hsiu-Jy Ho e Wei-Ming Lin. Task scheduling for multiprocessor systems with autonomous performance-optimizing control. J. Inf. Sci. Eng., 26(2):347-361, 2010. 83

[Hoo05] Han Hoogeveen. Multicriteria scheduling. European Journal of Operational Research, pages 592-623, 2005. 16 
[HS87] Dorit S. Hochbaum e David B. Shmoys. Using dual approximation algorithms for scheduling problems theoretical and practical results. J. ACM, 34:144-162, January 1987. 20

[IDE ${ }^{+}$06] Alexandru Iosup, Catalin Dumitrescu, Dick Epema, Hui Li e Lex Wolters. How are real grids used? the analysis of four grid traces and its implications. In Proceedings of the 7th IEEE/ACM International Conference on Grid Computing, GRID '06, pages 262-269, Washington, DC, USA, 2006. IEEE Computer Society. 55

[IPC ${ }^{+}$09] Michael Isard, Vijayan Prabhakaran, Jon Currey, Udi Wieder, Kunal Talwar e Andrew Goldberg. Quincy: fair scheduling for distributed computing clusters. In Proceedings of the ACM SIGOPS 22nd symposium on Operating systems principles, SOSP '09, pages 261-276, New York, NY, USA, 2009. ACM. 21

[JSC01] David B. Jackson, Quinn Snell e Mark J. Clement. Core algorithms of the maui scheduler. In Revised Papers from the 7th International Workshop on Job Scheduling Strategies for Parallel Processing, JSSPP '01, pages 87-102, London, UK, UK, 2001. Springer-Verlag. 27, 71

[KPS13] Ian Kash, Ariel D. Procaccia e Nisarg Shah. No agent left behind: dynamic fair division of multiple resources. In Proceedings of the 2013 international conference on Autonomous agents and multi-agent systems, AAMAS '13, pages 351-358, Richland, SC, 2013. International Foundation for Autonomous Agents and Multiagent Systems. 21

$\left[\mathrm{KSS}^{+}\right.$05] Rajkumar Kettimuthu, Vijay Subramani, Srividya Srinivasan, Thiagaraja Gopalsamy, D. K. Panda e P. Sadayappan. Selective preemption strategies for parallel job scheduling. Int. J. High Perform. Comput. Netw., 3(2/3):122-152, November 2005. 47

[LC91] Keqin Li e Kam-Hoi Cheng. Job scheduling in a partitionable mesh using a 
two-dimensional buddy system partitioning scheme. Parallel and Distributed Systems, IEEE Transactions on, 2(4):413-422, 1991. 83

[Lee91] Chung-Yee Lee. Parallel machines scheduling with nonsimultaneous machine available time. Discrete Appl. Math., 30:53-61, January 1991. 58

[LKB77] Jan Karel Lenstra, AHG Rinnooy Kan e Peter Brucker. Complexity of machine scheduling problems. 1977. 11, 12

[LSV06] Arnaud Legrand, Alan Su e Frédéric Vivien. Minimizing the stretch when scheduling flows of biological requests. In Proceedings of the eighteenth annual ACM symposium on Parallelism in algorithms and architectures, SPAA '06, pages 103-112, New York, NY, USA, 2006. ACM. 24, 79

[MF12] David Mehrzadi e Dror G. Feitelson. On extracting session data from activity logs. In Proceedings of the 5th Annual International Systems and Storage Conference, SYSTOR '12, pages 3:1-3:7, New York, NY, USA, 2012. ACM. 68

[Pap78] C.H. Papadimitriou. Scheduling Interval-ordered Tasks. TR // Center for Research in Computing Technology, Harvard University. Center for Research in Computing Techn., Aiken Computation Laboratory, Univ., 1978. 70

[Pro13] Ariel D. Procaccia. Cake cutting: not just child's play. Commun. ACM, 56(7):7887, July 2013. 4, 21

[PRT12] Vinicius Pinheiro, Krzysztof Rzadca e Denis Trystram. Campaign scheduling. In IEEE International Conference on High Performance Computing (HiPC), Proceedings, pages 1-10, 2012. 38

[RLAI04] David Raz, Hanoch Levy e Benjamin Avi-Itzhak. A resource-allocation queueing fairness measure. SIGMETRICS Perform. Eval. Rev., 32(1):130-141, June 2004. 21,27

[SF06] E. Shmueli e D.G. Feitelson. Using site-level modeling to evaluate the performance of parallel system schedulers. In Modeling, Analysis, and Simulation of 
Computer and Telecommunication Systems, 2006. MASCOTS 2006. 14th IEEE International Symposium on, pages 167 - 178, sept. 2006. 68

[Sit] TOP500 Supercomputer Sites. Development over time. http://www.top500. org/statistics/overtime/. Last accessed on 30 Aug, 2013. 1

[SKS04] Gerald Sabin, Garima Kochhar e P. Sadayappan. Job fairness in nonpreemptive job scheduling. In Proceedings of the 2004 International Conference on Parallel Processing, ICPP '04, pages 186-194, Washington, DC, USA, 2004. IEEE Computer Society. 21, 27

[SKSS02] S. Srinivasan, R. Kettimuthu, V. Subramani e P. Sadayappan. Characterization of backfilling strategies for parallel job scheduling. In Parallel Processing Workshops, 2002. Proceedings. International Conference on, pages 514-519, 2002. 27

[SLL $\left.{ }^{+} 49\right]$ Samuel A Stouffer, Arthur A Lumsdaine, Marion Harper Lumsdaine, Robin M Williams Jr, M Brewster Smith, Irving L Janis, Shirley A Star e Leonard S Cottrell Jr. The american soldier: combat and its aftermath.(studies in social psychology in world war ii, vol. 2.). 1949. 3

[SS05] Gerald Sabin e P. Sadayappan. Unfairness metrics for space-sharing parallel job schedulers. In Proceedings of the 11th international conference on Job Scheduling Strategies for Parallel Processing, JSSPP'05, pages 238-256, Berlin, Heidelberg, 2005. Springer-Verlag. 21, 44

[ST09] Erik Saule e Denis Trystram. Multi-users scheduling in parallel systems. In Proc. of IEEE International Parallel and Distributed Processing Symposium 2009, pages 1-9, Washington, DC, USA, may 2009. 17, 18, 19, 20, 38, 42

[Try12] Denis Trystram. Les riches heures de l'ordonnancement. TSI (Revue des sciences et technologies de línformation), 31(8-10):1021-1047, 2012. 12

[Ull75] J. D. Ullman. Np-complete scheduling problems. J. Comput. Syst. Sci., 10(3):384-393, June 1975. 12 
[Val90] Leslie G. Valiant. A bridging model for parallel computation. Commun. ACM, 33(8):103-111, 1990. 33

[VC05] Sangsuree Vasupongayya e Su-Hui Chiang. On job fairness in non-preemptive parallel job scheduling. In IASTED PDCS, pages 100-105, 2005. 21

[Voo03] Mark Voorneveld. Characterization of pareto dominance. Operations Research Letters, 31:7-11, january 2003. 16

[WC01] Yiqiong Wu e Guohong Cao. Stretch-optimal scheduling for on-demand data broadcasts. In Computer Communications and Networks, 2001. Proceedings. Tenth International Conference on, pages 500 -504, 2001. 24

[YJG03] AndyB. Yoo, MorrisA. Jette e Mark Grondona. Slurm: Simple linux utility for resource management. In Dror Feitelson, Larry Rudolph e Uwe Schwiegelshohn, editors, Job Scheduling Strategies for Parallel Processing, volume 2862 of Lecture Notes in Computer Science, pages 44-60. Springer Berlin Heidelberg, 2003. 26, 27, 92

[Zah] Matei Zaharia. The hadoop fair scheduler. http://hadoop.apache.org/docs/ r1.1.2/fair_scheduler.html. Last accessed on 22 April, 2012. 27

[ZAT05] Julia Zilber, Ofer Amit e David Talby. What is worth learning from parallel workloads?: a user and session based analysis. In Proceedings of the 19th annual international conference on Supercomputing, ICS '05, pages 377-386, New York, NY, USA, 2005. ACM. 5

[ZF12] Netanel Zakay e Dror G. Feitelson. On identifying user session boundaries in parallel workload logs. In Proc. of the 16th Workshop on Job Scheduling Strategies for Parallel Processing, The Hebrew University, Israel, may 2012. 5, 68, 71 